San Jose State University

SJSU ScholarWorks

Master's Theses

Master's Theses and Graduate Research

1993

\title{
New approach for the preparation of a hydride-modified substrate as an intermediate in the synthesis of surface-bonded materials
}

Chao-Hau Chu

San Jose State University

Follow this and additional works at: https://scholarworks.sjsu.edu/etd_theses

\section{Recommended Citation}

Chu, Chao-Hau, "New approach for the preparation of a hydride-modified substrate as an intermediate in the synthesis of surface-bonded materials" (1993). Master's Theses. 618.

DOI: https://doi.org/10.31979/etd.k8qc-c6uf

https://scholarworks.sjsu.edu/etd_theses/618

This Thesis is brought to you for free and open access by the Master's Theses and Graduate Research at SJSU ScholarWorks. It has been accepted for inclusion in Master's Theses by an authorized administrator of SJSU ScholarWorks. For more information, please contact scholarworks@sjsu.edu. 


\section{INFORMATION TO USERS}

This manuscript has been reproduced from the microfilm master. UMI films the text directly from the original or copy submitted. Thus, some thesis and dissertation copies are in typewriter face, while others may be from any type of computer printer.

The quality of this reproduction is dependent upon the quality of the copy submitted. Broken or indistinct print, colored or poor quality illustrations and photographs, print bleedthrough, substandard margins, and improper alignment can adversely affect reproduction.

In the unlikely event that the author did not send UMI a complete manuscript and there are missing pages, these will be noted. Also, if unauthorized copyright material had to be removed, a note will indicate the deletion.

Oversize materials (e.g., maps, drawings, charts) are reproduced by sectioning the original, beginning at the upper left-hand corner and continuing from left to right in equal sections with small overlaps. Each original is also photographed in one exposure and is included in reduced form at the back of the book.

Photographs included in the original manuscript have been reproduced xerographically in this copy. Higher quality $6 " \mathrm{x}$ " 9 " black and white photographic prints are available for any photographs or illustrations appearing in this copy for an additional charge. Contact UMI directly to order.

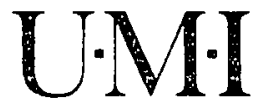

University Microfilms Internatıonal A Bell \& Howell Iniormation Company 300 North Zeeb Road. Ann Arbor. MI 48106-1346 USA 313:761-4700 800:521-0600 
Order Number 1354193

New approach for the preparation of a hydride-modified substrate as an intermediate in the synthesis of surface-bonded materials

\author{
Chu, Chao-Hau, M.S.
}

San Jose State University, 1993

Copyright (C1993 by Chu, Chao-Hau. All rights reserved.

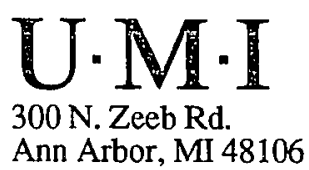




\title{
NEW APPROACH FOR THE PREPARATION OF A HYDRIDE- MODIFIED SUBSTRATE AS AN INTERMEDIATE IN THE SYNTHESIS OF SURFACE-BONDED MATERIALS
}

\author{
A Thesis \\ Presented to \\ the Faculty of the Department of Chemistry \\ San Jose State University
}

\author{
In Partial Fulfillment \\ of the Requirements for the Degree \\ Master of Science
}

by

Chao-Hau Chu

August, 1993 
APPROVED FOR THE DEPARTMENT OF CHEMISTRY

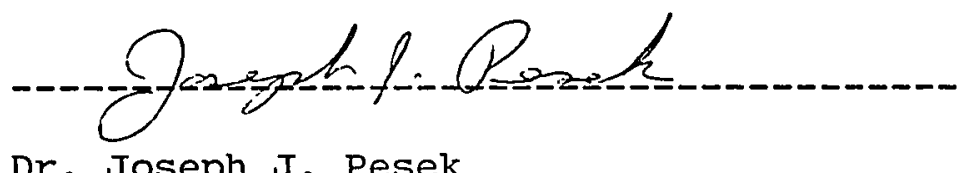

Dr. Joseph J. Pesek

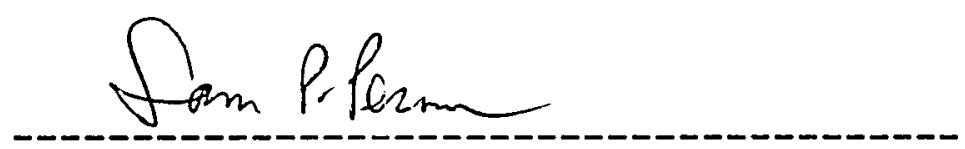

Dr. Sam P. Perone

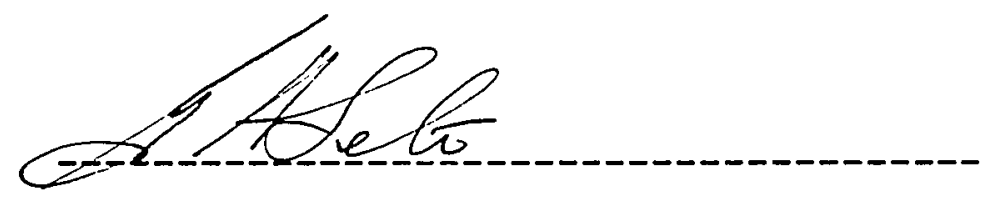

Dr. Gerald A. Selter

APPROVED FOR THE UNIVERSITY

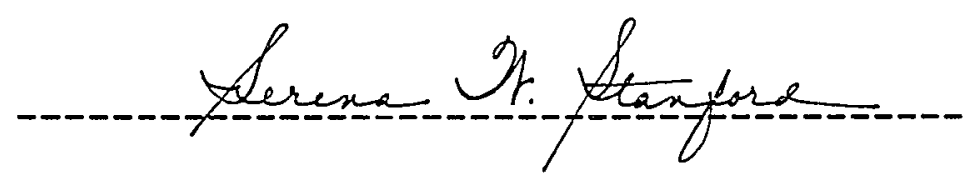


(C) 1993

Chao-Hau Chu

ALL RIGHTS RESERVED 


\section{ABSTRACT}

\section{NEW APPROACH FOR THE PREPARATION OF \\ A HYDRIDE-MODIFIED SUBSTRATE AS AN INTERMEDIATE \\ IN THE SYNTHESIS OF SURFACE-BONDED MATERIALS}

\section{by Chao-Hau Chu}

A new approach to produce a hydride-modified support which serves as an intermediate for the synthesis of chromatographic bonded phases is investigated. The procedure involves the controlled deposition of the hydrolysis product of triethoxysilane (TES) or related silanes to preferentially form a $\mathrm{Si}-\mathrm{H}$ monolayer on inorganic oxide substrates such as silica. Chemical, spectroscopic as well as thermal analysis are employed for the characterization of the intermediate. In addition to its greater simplicity, hydrosilanization with TES provides superior SiH coverages as compared to previously reported chlorination/reduction approach.

A number of commercially available silica substrates were subjected to an optimum hydrosilanization procedure and the results were compared with those obtained using a chlorination/reduction sequence. These hydride-modified substrates where further derivatized with 1-octene in presence of a platinum catalyst. The octyl-bonded phases obtained were shown to have good ligand density which should render them suitable for HPLC applications. 
I would like to show my sincere appreciation to my research advisor, Dr. Joseph J. Pesek, for his constant guidance and advice during my research.

I would like to extend my sincere gratitude to the members of my thesis committee, Dr. Sam P. Perone and Dr. Gerry A. Selter, for their time, advice and suggestions in my research.

Special thanks are also extended to Dr. Junior Sandoval for his constant advice and helps during my research.

Finally, I would like to thank my parents for their devotion and encouragement throughout the course of my graduate study. 
Acknowledgements $\ldots \ldots \ldots \ldots \ldots \ldots \ldots \ldots \ldots \ldots \ldots \ldots$ iv

List of Tables .......................... vii

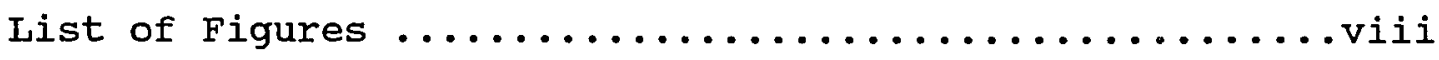

1. Introduction $\ldots \ldots \ldots \ldots \ldots \ldots \ldots \ldots \ldots \ldots \ldots \ldots \ldots \ldots$

1.1. Background $\ldots \ldots \ldots \ldots \ldots \ldots \ldots \ldots \ldots \ldots \ldots \ldots$

1.2. Aims of This Work ................... 7

2. Experimental .......................... 9

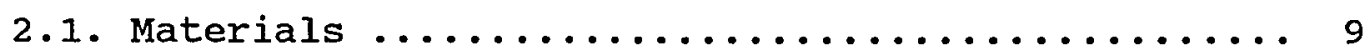

2.2. Instrumentation $\ldots \ldots \ldots \ldots \ldots \ldots \ldots \ldots \ldots$

2.2.1. Spectrometry $\ldots \ldots \ldots \ldots \ldots \ldots \ldots \ldots$

2.2.2. Thermoanalytical Techniques ............ 11

2.2.3. Gas Chromatography ............... 12

2.2.4. Elemental Analysis ............... 12

2.3. Procedures ......................... 13

2.3.1. Silica Modifications .............. 13

2.3.1.1. Harosilanization with TES .........13

2.3.1.1.1. Ethanol as a Solvent ..........13

2.3.1.1.2. Dioxane as a Solvent .......... 14

2.3.1.2. Chlorination/Reduction ........... 14

2.3.1.3. Olefin Hydrosilation ............ 15

2.3.2. Analytical Procedures .............. 16

2.3.2.1. Hydrogen Evolution/Gas Volumetry ..... 16 
2.3.2.2. Hydrogen Evolution/Gas Chromatography . 17 2.3.2.3. Mercury Reduction Method .......... 19

2.3.3. Long-Term Hyarolysis Test ............. 19

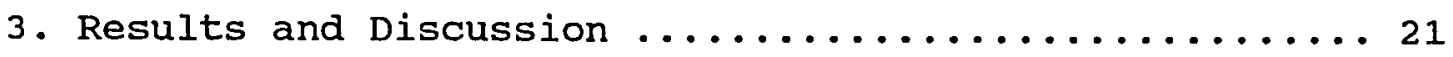

3.1. Preliminary work ..................... 24

3.2. Hydrosilanization in an Aprotic Solvent .......46 46

3.2.1. Solvent and Temperature Effect .........46

3.2.2. Effect of TES Concentration .......... 51

3.2.3. Effect of Reaction Time ........... 56

3.2.4. Effect of Acid Catalyst ............. 59

3.2.5. Suggested Experimental Conditions for the Hydrosilazation of Silica with TES ...... 64

3.2.6. TGA Profiles of Hydrosilanized Silica .....66 66

3.3. Application of Hydrosilanization with TES on Several Commercial silicas ............... 71

3.4. Bonded Phases from Hydrosilanized silicas ...... 93

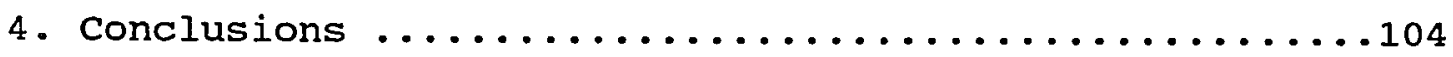

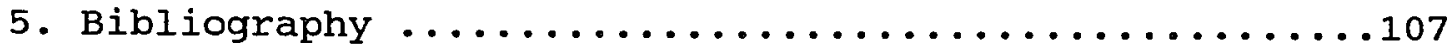

6. Appendix ...............................110

6.1. Significance of Hydrosilane Coverage in a Hydride-Intermediate support .............110

6.2. Hydrogen Displacement Methods .............. 112

6.3. Correlation Between Hydrosilane Coverage and Dsc/Air Data ............................114 


\section{IIST OF TABLES}

Page

Table 1. Silica Support Materials Used in This Work .... 10

Table 2. DSC/Air Data for Hydrosilanized Partisil-40 ... 33

Table 3. Effect of TES Concentration on the Thermooxidation and Hydrosilane Content of Hydrosilanized Silicas (DSC/Air) $\ldots \ldots \ldots \ldots \ldots \ldots \ldots \ldots \ldots \ldots$

Table 4. Effect of Solvent and Reaction Temperature on the TES Hydrosilanization of Partisil-40 .....50

Table 5. Effect of TES Concentration on Silica Hydrosilanization ...................... 56

Table 6. Effect of Reaction Time on Hydrosilanization with TES Carried out in Dioxane Solvent ...... 59

Table 7. Physicochemical Characteristics of Native Sizicas Used for Hydride-Modification ......... 72

Table 8. Physicochemical Characteristics of HydrideModified Silicas Prepared via Chlorination/ Reduction Sequence $\ldots \ldots \ldots \ldots \ldots \ldots \ldots \ldots$

Table 9. Physicochemical Characteristics of HydrideModified Silicas Prepared via Hydrosilanization

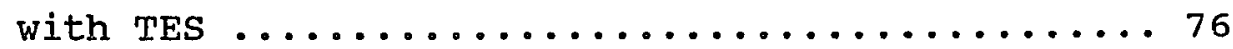

Table 10. Surface Coverage Properties of Octyl-Silicas Prepared via Hydrosilation of 1-Octene .......100 


\section{LIST OF FIGURES}

Page

Figure 1. Glass Tube for Hydrogen Gas Collection ...... 18

Figure 2. Partial DRIFT Spectra of the Products of TES Hydrosilanization of silica ............ 26

Figure 3. Partial DRIFT Spectra of the Products of TES Hydrosilanization of Silica ............ 27

Figure 4. Partial DRIFT Spectra of Native Partisil-40 Silica and its Hydrosilanization Products .... 28

Figure 5. DSC Thermograms of the Products of Silica Hydrosilanization with TEs $\ldots \ldots \ldots \ldots \ldots \ldots$

Figure 6. Effect of Reaction Time on DSC Thermogrames in

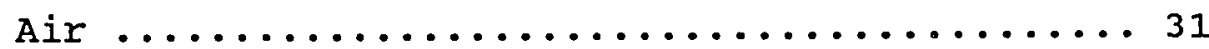

Figure 7. Effect of TES Concentration and Reaction Time on the oxidation Enthalpy of Hydrosilanized Partisil-40 as Measured by DSC/Air ........ 32

Figure 8. TGA/Air Curves for the Thermooxidation of Hydroilanized Partisil-40 ..................... 34

Figure 9. TGA/Nitrogen (or Argon) Curves for Hydrosilan-

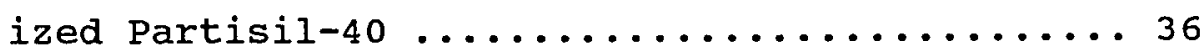

Figure 10. Specific Hydrosilane Coverage as a Function of TES Concentration and Reaction 'rime ..... 37

Figure 11. DSC Thermograms of the TES Polycondensation Product in the Absence of Silica substrate ... 39 viii 
Figure 12. Partial DRIFT Spectra (Si-H Stretching Band) of Hydrosilanization Products ........... 40

Figure 13. Partial DRIFT Spectra (Si-H Streching Band) of Hydrosilanized silica .............. 42

Figure 14. DSC Thermograms of Hydrosilanized Partisil-40. 43

Figure 15. Effect of TES Concentration on SiH Coverage .. 45 Figure 16. Partial DRIFT Spectra (Si-H Stretching Band) of Silica Hydrosilanization Products ......4 47

Figure 17. DSC Thermograms of TES on Silica ......... 49

Figure 18. Effect of TES Concentration on SiH Coverage, Dioxane Solvent .................. 53

Figure 19. Effect of TES Concentration on the DSC Thermooxidation of Hydrosilanized silica ....... 54

Figure 20. Effect of TES Concentration on the DSC Oxidation Enthalpy, H, Dioxane Solvent ........ 55

Figure 21. Effect of Reaction Time on $\mathrm{SiH}$ Coverage ..... 57 Figure 22. Effect of Reaction Time on DSC Thermograms ... 58 Figure 23. Effect of $\mathrm{HCl}$ Concentration on Hydrosilane Coverage $\ldots \ldots \ldots \ldots \ldots \ldots \ldots \ldots \ldots \ldots \ldots \ldots \ldots \ldots \ldots$

Figure 24. Partial DRIFT Spectra (Si-H Streching Band) of Hydrosilanized silica under Varying $\mathrm{HCl}$

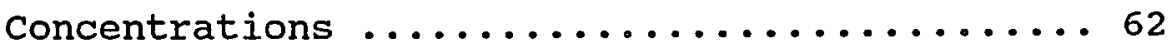

Figure 25. DSC Thermograms of Hydrosilanized Silica at Various HCl Concentrations .............6 63 ix 
Figure 26. DSC Thermograms of Hydrosilanized Silica at Various $\mathrm{HCl}$ Concentrations .............65

Figure 27. TGA/Argon Curves for Hydrosilanized Partisil-40 .......................68

Figure 28. Partial DRIFT Spectra of Hydrosilanized silica ........................69

Figure 29. Specific Hydrosilane coverages for Several Commercial silicas Subjected to Two HydrideModification Methods ................ 74

Figure 30. Hydrosilane Surface Density for Several Commercial silicas subjected to Two HydrideModification Methods .................. 75

Figure 31. DSC/Air Oxidation Curves of Hydride-Modified Partisil-40 silica .................. 78

Figure 32. DSC/Air Oxidation Curves of Hydride-Modified Partisil-10 silica .................. 79

Figure 33. DSC/Air Oxidation Curves of Hydride-Modified Kromasil silica .................... 80

Figure 34. DSC/Air Oxidation Curves of Hydride-Modified Vydac-lot 890414 silica ............... 81

Figure 35. DSC/Air Oxidation Curves of Hydride-Modified

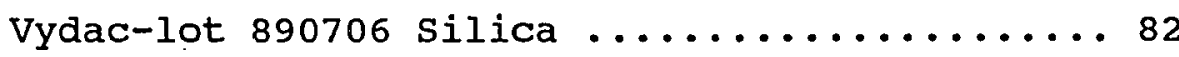

Figure 36. DSC/Air Oxidation Curves of Hydride-Modified Nucleosil silica .................. 83 
Figure 37. Partial DRIFT Spectra of Hydride-Modified Partisil-40 silicas ................ 84

Figure 38. Partial DRIFT Spectra of Hydride-Modified Partisil-10 silicas .................... 85

Figure 39. Partial DRIFT Spectra of Hydride-Modified Kromasil silicas ................. 86

Figure 40. Partial DRIFT Spectra of Hydride-Modified Vydac (Lot 890414 ) silicas ............... 87

Figure 41. Partial DRIFT Spectra of Hydride-Modified Vydac (Lot 890706) Silicas ............. 88

Figure 42. Partial DRIFT Spectra of Hydride-Modified Nucleosil silicas ................... 89

Figure 43. Effect of Hydride-Modification on Specific Surface Area for Several Commercial silica Supports $\ldots \ldots \ldots \ldots \ldots \ldots \ldots \ldots \ldots \ldots \ldots \ldots \ldots$

Figure 44. Effect of Hydride-Modification on Pore Size for Several commercial silica supports ..... 92

Figure 45. Change in the Partial DRIFT Spectra of Partisil-40 silica upon Derivatization .........994

Figure 46. Change in the Partial DRIFT Spectra of Partisil-10 Silica upon Derivatization ......... 95

Figure 47. Change in the Partial DRIFT Spectra of Kromasil Silica upon Derivatization .......... 96

Figure 48. Change in the Partial DRIFT Spectra of Vydac xi 
(Lot 890414 ) Silica upon Derivatization ..... 97

Figure 49. Change in the Partial DRIFT Spectra of Vydac (Lot 890706) Silica upon Derivatization ..... 98

Figure 50. Change in the Partial DRIFT Spectra of Nucleosil Silica upon Derivatization ........ 99

Figure 51. Surface Coverage of Octyl-bonded Vydac Silica as a Function of Hydrolysis Time ..........102

Figure 52. Relative Coverage as a Function of Hydrolysis

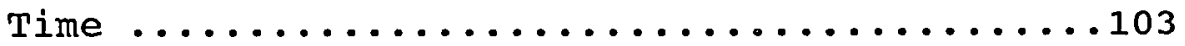

Figure 53. Correlation Between DSC/Air Data and Specific Hydrosilane Coverage ..................116 


\section{INTRODUCTION}

\subsection{Background}

High Performance Liquid Chromatography (HPLC) is a very powerful separation technique which has shown a dramatic growth in relatively recent years. Because the nature of the support used as the stationary phase can strongly affect the efficiency of separations, the development of new approaches for producing both covalently-bonded, silica-based supports and polymeric organic stationary phases has received most of the attention.

Several synthetic approaches have been developed to covalently attach organic moieties onto the silica surface. Currently, the most common approach is a silanization procedure by which fully hydroxylated silica is reacted with a triorganochlorosilane to form a monolayer of triorganosiloxane-type linkages:

$\equiv \mathrm{Si}-\mathrm{OH}+\mathrm{XSiR}^{\prime}{ }_{2} \mathrm{R}-----\rightarrow \equiv \mathrm{Si}-\mathrm{O}-\mathrm{SiR}^{\prime}{ }_{2} \mathrm{R}+\mathrm{HX}$

where $\mathrm{R}^{\prime}$ is almost always a methyl $\left(\mathrm{CH}_{3}{ }^{-}\right)$group, $\mathrm{X}$ is an easily hydrolyzable group such as halide, alkoxy, acyloxy, etc. and $R$ has the general formula $C_{n} H_{2 n+1}$, with $n=1$ to about 20 , more commonly, 8 and 18 .

Some limitations of triorganosiloxane-type linkages 
exist. First, the steric hindrance of the triorganosilyl group causes a relatively limited coverage of the organic moieties on the silica surface (1). As a result, a significant fraction of silanol ( $\mathrm{SiOH}$ ) groups remains unreacted after the reaction. These remaining silanols may lead to very strong interactions with the species under separation, particularly with basic solutes $(2,3)$. Additionally, the relatively poor hydrolytic stability of Si-O-Si-c linkages is an undesirable feature of currently available bonded supports (3).

In addition to the widely-used monomeric alkyldimethylsiloxanes (equation 1) bonded phases have been prepared with trifunctional organosilanes of the type $\mathrm{RSix}_{3}$, in the presence of a measured amount of water and a suitable solvent $(1,4):$

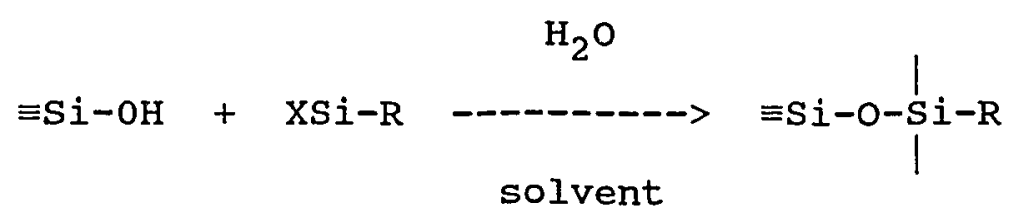

The resulting bonded material contains an organic layer whose thickness varies depending on reaction conditions. Although increased alkyl densities are usually obtained with these packings, they exhibit a tendency to contribute additional silanols to the bonded phase because, due to the steric hindrance arising from the R-group, hydroxyl species 
formed upon hydrolysis of the si-x linkages are not completely condensed. Moreover, such packings often show limited long-term hydrolytic stability, particularly when aggressive mobile phases are used.

As a result of the forementioned limitations of organosiloxane-type bonded phases, new approaches involving the formation of surface Si-C linkages have been proposed $(5,6)$. A direct Si-C linkage can provide a denser coverage as well as a more hydrolytically stable bonded phase because (i) the two bulky methyl groups, which hinder the access of the triorganochlorosilane to all the available surface silanol groups, will no longer be present; and (ii) the Si-C linkage is more stable than the trialkylsiloxane linkage. The reaction of chlorinated silica with organometallic compounds has been a common way to form Si-C linkages on the surface. Chlorination of silica can be carried out by the reaction between silica and a chlorinating reagent such as thionyl chloride (1):

$$
\begin{aligned}
& \text { toluene } \\
& \equiv \mathrm{Si}-\mathrm{OH}+\mathrm{SOCl}_{2}-\cdots-\longrightarrow \rightarrow \mathrm{Si}-\mathrm{Cl}+\mathrm{HCl}+\mathrm{SO}_{2}
\end{aligned}
$$

Grignard reagents and organolithium compounds are two common types of organometallic compounds used as alkylating re- 
agents $(5,6)$ :

$$
\begin{aligned}
& \text { ESi-Cl }+\mathrm{R}-\mathrm{M}----->\quad \mathrm{Si}-\mathrm{R}+\mathrm{MCl} \\
& \text { where }-\mathrm{M}=-\mathrm{Ii} \text { or }-\mathrm{MgBr}
\end{aligned}
$$

An alternate approach for producing si-C linkages on silica supports has been recently proposed by Sandoval and Pesek (7). In this approach an intermediate silica support containing silicon hydride $(\mathrm{Si}-\mathrm{H})$ surface groups is produced and then reacted with an olefin to form an addition product on the silica surface. The bonding reaction, also known as hydrosilylation or hydrosilation, involves the catalytic addition of the surface silicon hydride species to a terminal double bond:

$$
\begin{aligned}
& \text { Pt-cat } \\
& \equiv \mathrm{Si}-\mathrm{H}+\mathrm{H}_{2} \mathrm{C}=\mathrm{CH}-\mathrm{R}-\cdots---->\quad \equiv \mathrm{Si}-\mathrm{CH}_{2} \mathrm{CH}_{2}-\mathrm{R}
\end{aligned}
$$

Since it involves the formation of direct si-C linkages, this approach should provide an extensive coverage of the attached groups. An obvious requirement for this approach is that silicon hydride (hydrosilane) species must be already present on the silica surface before the actual attachment of the organic group takes place.

Basically, two different approaches for producing such 
a hydride intermediate support can be distinguished: (i) The direct preparation of a polyhydrosiloxane (PHS) gels which have a chemical composition corresponding to $\left(\mathrm{HSiO}_{3 / 2}\right)_{\mathrm{n}}$ and (ii) The chemical derivatization of a commercially available silica gel support whose surface silanol groups are chemically converted to hydrosilane groups.

The PHS gel can be prepared by the polycondensation of the hydrolysis product of a substituted silane of the type HSix $_{3}$, in the presence of an inorganic acid catalyst:

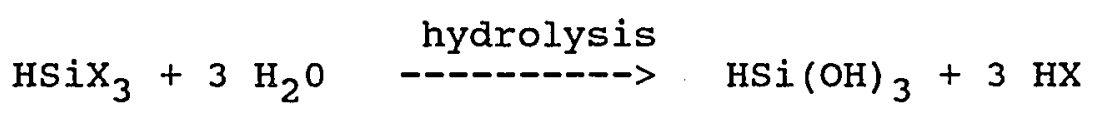

polycondensation

$$
n \mathrm{HSi}(\mathrm{OH})_{3}-\cdots\left(\mathrm{HSiO}_{3 / 2}\right)_{\mathrm{n}}+3 / 2 \mathrm{n} \mathrm{H}_{2} \mathrm{O}
$$

Although the physical and chemical characteristics (pore size, specific surface area, thermal and hydrolytic stability, etc.) of these PHS gels have been thoroughly studied (8-13), the lack of commercial PHS gels precludes the immediate utilization of these materials. On the other hand, the commercial availability of a variety of silicas with well-characterized geometries along with the relatively 
simple chemistry involved in their hydride derivatization (vide infra) make the second approach an attractive choice.

Two different approaches have been investigated for the hydride derivatization of fully hydroxylated silicas: (i) Chemisorption of hydrogen from a pyrolytically activated silica which had been previously methylated $(14,15)$. The high temperature required for the pyrolysis process (about $800^{\circ} \mathrm{C}$ ) might result in an extensive deterioration of the pore structure of the support, therefore precluding this approach as a method for the preparation of the hydride intermediate for chromatographic purposes. (ii) Reduction with an inorganic hydride on a silica surface whose silanol groups have been previously converted to Sicl groups (7):

$$
\equiv \mathrm{Si}-\mathrm{OH}+\mathrm{SOCl}_{2} \frac{\text { toluene }}{\text { reflux }} \equiv \mathrm{Si}-\mathrm{Cl}+\mathrm{SO}_{2}+\mathrm{HCl} \text { (chlorination) }
$$

$$
\equiv \mathrm{Si}-\mathrm{Cl} \underset{\mathrm{LiAlH} 4}{\text { ether }} \quad \equiv \mathrm{Si}-\mathrm{H}
$$$$
\text { (reduction) }
$$

One major drawback of this approach is its extreme sensitivity to moisture during both the chlorination and the reduction steps. Additionally, it involves time-consuming 
procedures, particularly during the chlorination step. Moreover, the formation of relatively volatile reduction byproducts requires the use of a dry-ice condenser (or equivalent) which further complicates the experimental setup of this approach. It is therefore necessary to devise an alternate method for preparing a hydride-intermediate support which overcomes the forementioned limitations of the chlorination/reduction sequence (equations 8 and 9). The development of such an alternative approach is a major subject of this work.

1.2. Aims of This Work

The present research has several fundamental objectives. One major goal is the development of a new approach to produce a hydride-derivatized silica support for which the synthesis limitations described above are reduced or eliminated. Another objective of this research is to fully evaluate the new hydride intermediate in terms of its spectroscopic, thermal and chemical properties. A thorough comparison of the new hydride product with that obtained from a chlorination/reduction procedure (7) is also an aim of this research. Finally, this work was also pointed at developing a suitable analytical method to determine surface silane coverage on the hydride intermediate. 
The new synthetic procedure involves the controlled chemisorption of hydrosilanetriol $\mathrm{HSi}(\mathrm{OH})_{3}$, on an inorganic substrate (silica). Hydrosilanetriol is the hydrolysis product of hydrosilanes of the general formula $\mathrm{HSix}_{3}$, where $\mathrm{X}$ is a hydrolytically labile group. The silanetriol becomes covalently attached to the inorganic substrate by means of condensation reactions of the type

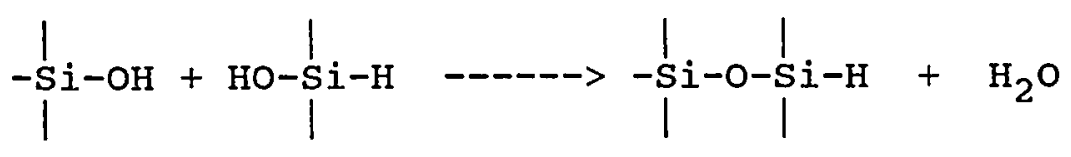

It is expected that under certain experimental conditions, a monolayer of $\mathrm{SiH}$ species is produced by the proposed method. This new approach is also expected to provide several advantages when compared to the previously investigated chlorination/reduction sequence: (i) Shorter process time, since the reaction does not require time-consuming procedures such as those associate with chlorination and reduction of silicas. (ii) A simpler reaction procedure, since no moisture-free conditions are required. In fact, the presence of water is necessary for the initial hydrolysis of the $\mathrm{HSix}_{3}$ material to occur. (iii) similar to other silanol polycondensation processes in acidic media (16), deposition of hydrosilanetriol is expected to produce a monolayer with a maximum of siloxane linkages and a minimum of uncondensed silanols. 


\section{EXPERIMENTAL}

\subsection{Materials}

Ethanol (95\%, Gold Shield Chemical Co., Hayward, CA) was used as received. p-Dioxane (J. T. Baker Chemical Co., Phillipsburg, NJ) was aried by contact with calcium hydride (Sigma Chemical Co., st. Louis, MO) for several days, and then was distilled right before use. Triethoxysilane (Petrarch Systems Silanes \& Silicons, Bristol, PA) was used as the hydrosilane reagent. Potassium bromide (Harshaw/Filtrol Partnership, Solon, OH) was employed for the IR spectra. Triphenylsilane (Sigma Chemical Co., St. Louis, MO) was used as standard for the determination of hydrosilane coverage. Dionized water was obtained from a Milli-Q water purification system (Millipore Corp. Bedford, MA). The silica support materials used and their major physical properties are shown in Table 1.

\subsection{Instrumentation}

\subsubsection{Spectrometry}

Diffuse reflectance infrared Fourier transform (DRIFT) spectra were taken with a Perkin-Elmer model 1800 FT-IR 
Table 1. Silica Support Materials Used in This Work

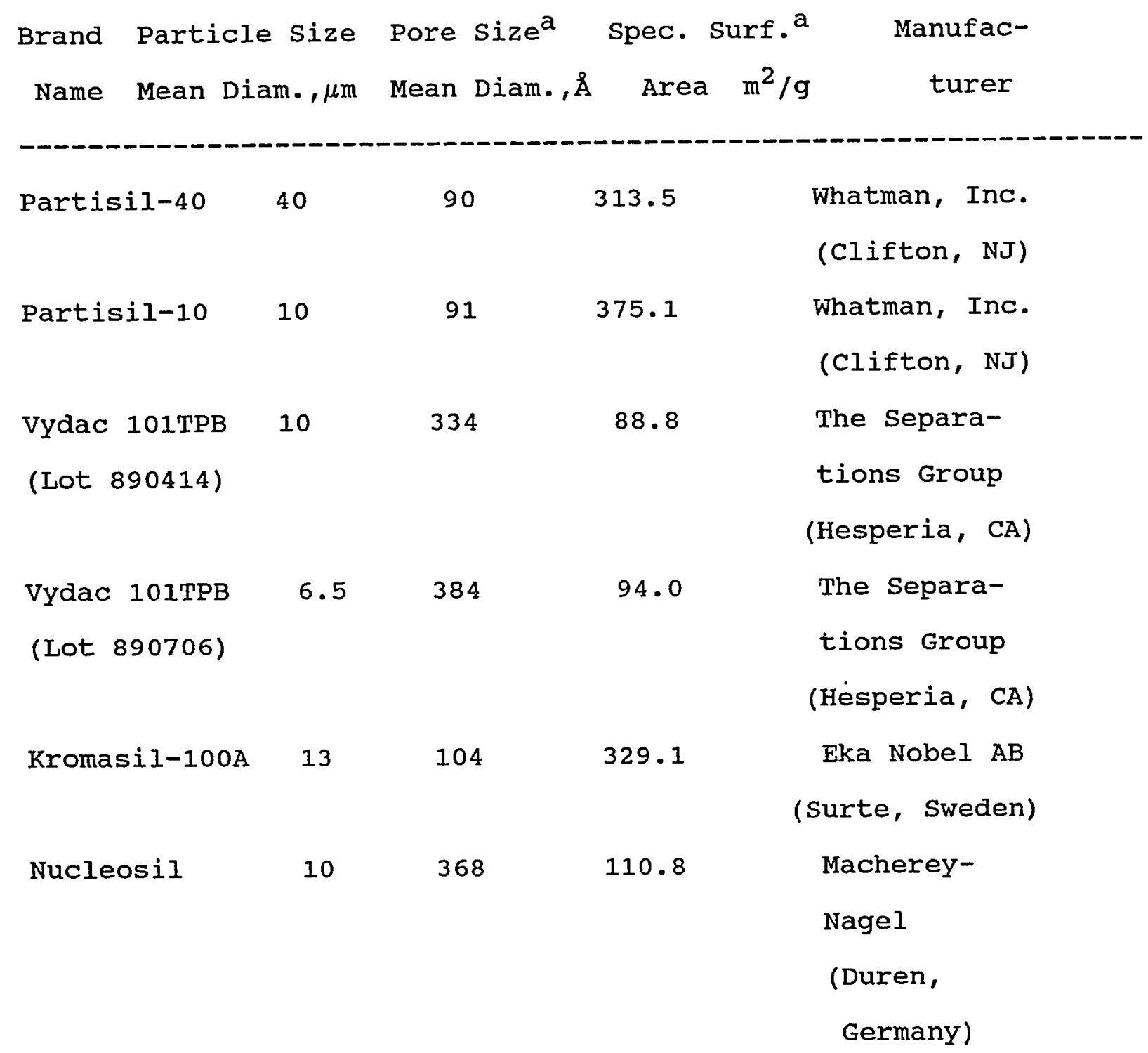

a BET, nitrogen adsorption method 
spectrometer equipped with a deuterated triglycine surfate (DTGS) detector. The silica sample was mixed with an equal amount of pure $\mathrm{KBr}$ which was ground in an agate morter and pre-dried in an oven at $110^{\circ} \mathrm{C}$ for at least 24 hours. The sample mixture was filled into the DRIFT accessory's cup and a smooth surface was formed by using a microscope slide to gently press the cup. In order to obtain a maximum signal throughput, the height of the sample cup was adjusted by applying an alignment routine provided by Perkin-Elmer as standard software. Spectra were taken in the 4000-450 $\mathrm{cm}^{-1}$ region with a nominal resolution of $2 \mathrm{~cm}^{-1}$. One hundred sample scans were ratioed against pure $\mathrm{KBr}$ as reference. Spectra shown were normalized to $100 \%$ transmittance (7).

2.2.2. Thermoanalytical Techniques

Differential scanning calorimetric (DSC) thermograms were obtained under air atmosphere with a Perkin-Elmer Model DSC-7 instrument. Silica samples (5 to $10 \mathrm{mg}$ ) were loaded into a platinum pan and heated to $300^{\circ} \mathrm{C}$ until no heat exchange was observed. The temperature was then raised to $550{ }^{\circ} \mathrm{C}$ at a rate of $20^{\circ} \mathrm{C} / \mathrm{min}(7)$.

Thermogravimetric analysis (TGA) data were obtained by using a Perkin-Elmer Model TGS-2 analyzer. Silica sample (6 to $10 \mathrm{mg}$ ) were loaded into a platinum pan and heated to 110 
- $\mathrm{C}$ at a rate of $80^{\circ} \mathrm{C} / \mathrm{min}$. This temperature was held until all the moisture contained in the sample was eliminated as indicated by no deflection of the weight signal. Then the temperature was raised at a rate of $20{ }^{\circ} \mathrm{C} / \mathrm{min}$ to $900^{\circ} \mathrm{C}$. The temperature again was held until no weight loss could be detected. The TGA runs were performed under either air or an inert gas $\left(\mathrm{N}_{2}\right.$ or $\left.\mathrm{Ar}\right)(7)$.

\subsubsection{Gas Chromatography}

A Varian Aerograph model 204 GC, equipped with a Houston Instrument Omni Scribe chart recorder and a spectraPhysics integrator was employed to measure the amount of $\mathrm{H}_{2}$ gas evolved from a measured amount of hydride-silica sample. Gas-tight syringes ( $5 \mathrm{ml}$, Precision Sampling Corp., Baton Rouge, LO) equipped with push-bottom valve were used to inject gas samples into the instrument.

\subsubsection{Elemental Analysis}

Carbon analysis on bonded silicas was carried out with a Perkin-Elmer model $240 \mathrm{C}$ elemental analyzer equipped with a Perkin-Elmer model 56 recorder. Silica samples ranging from 5 to $15 \mathrm{mg}$ size were used. 
2.3. Procedures

2.3.1. Silica Modifications

2.3.1.1. Hydrosilanization with TES

Unless otherwise specified, Partisil-40 was used as the starting silica material.

2.3.1.1.1. Ethanol as a Solvent

Silica $(1.000 \mathrm{~g})$ was suspended in $35 \mathrm{~mL}$ of $95 \%$ ethanol containing $0.10 \mathrm{M} \mathrm{HCl}$. A measured amount of TES, HSi(OEt) 3 , was added and the suspension was magnetically agitated at room temperature for a fixed time. Once separated by centrifugation, the product was washed with five 10-mL portions of $95 \%$ ethanol, two 10-mL portions of $50: 50 \mathrm{v} / \mathrm{v}$ ether/ethanol and one 10-mL portion of diethyl ether. Each washing was followed by about 1 min manual mixing and 5 min centrifugation. The final product was dried in air at room temperature overnight and then in a vaccum oven at $110{ }^{\circ} \mathrm{C}$ overnight. 
2.3.1.1.2. Dioxane as a Solvent

Silica $(1.000 \mathrm{~g})$ was placed in a 3-neck, 25-mL round bottom flask equipped with a condenser, an addition funnel with equalizing tube, a heating mantle and a magnetic stirrer. A measured amount of pre-dried dioxane was then added, followed by one $\mathrm{mL}$ of (aqueous) $3.1 \mathrm{M} \mathrm{HCl}$ solution. The suspension was heated to about 70-80 ${ }^{\circ} \mathrm{C}$. Then a measured amount of $0.20 \mathrm{M}$ TES/dioxane solution was added dropwise into the solution. The volumes of dioxane and TES solution were adjusted so as to complete the addition in 10-15 min and to obtain a total liquid volume of $30 \mathrm{~mL}$. Once the addition of TES/dioxane solution was over, the mixture was gently refluxed for a certain time. The product was centrifuged and washed consecutively with two $20-\mathrm{mL}$ portions of 20:80 water/dioxane, two 20-mL portions of dioxane and two 20-mL portions of diethyl ether. Each washing was followed by about 1 min mixing and centrifugation. The final product was dried in air at room temperature overnight and then in a vaccum oven at $110^{\circ} \mathrm{C}$ overnight.

\subsubsection{Chlorination/Reduction (7)}

Dried silica $(5.00 \mathrm{~g})$ were suspended in $60 \mathrm{~mL}$ of predried toluene and $10 \mathrm{~mL}$ of thionyl chloride were added. The 
mixture was magnetically agitated under a gentle reflux for 48 hours. The product was then washed consecutively with eight $30-\mathrm{mL}$ portions of dry toluene. Each washing was followed by $15 \mathrm{~min}$ of magnetic stirring. Finally, the chlorinated silica was washed with one $30-\mathrm{mL}$ portion of dry diethyl ether, remaining in a final fresh ether aliquot.

A 70-mL portion of $0.2 \mathrm{M} \mathrm{LiAlH}_{4}$ solution in ether was slowly added to the chlorinated silica/ether solution. The reaction was allowed to proceed under a gentle reflux for about 2 hours. A dry-ice condenser was employed to safely condense relatively volatile reduction byproducts $\left(A \mathrm{H}_{\mathrm{n}} \mathrm{Cl}_{3-\mathrm{n}}, \mathrm{n}=1-3\right)$. The product was washed with eight 30-mL portions of dry ether and then dried at room temperature. The "reduced" silica product was then added to $50 \mathrm{~mL}$ of a $0.50 \mathrm{M} \mathrm{HCl}$ solution and magnetically stirred for 10-15 min. This acid treatment was carried out four more times, after which the solid was washed consecutively with $50-\mathrm{mL}$ portions of $\mathrm{H}_{2} \mathrm{O}: \mathrm{THF} 1: 1 \mathrm{~V} / \mathrm{V}(2 \mathrm{x}), \mathrm{THF}(2 \mathrm{x})$ and ethyl ether $(2 \mathrm{x})$. The final product was dried in air at room temperature and then in a vacuum oven at $110^{\circ} \mathrm{C}$ overnight.

2.3.1.3. Olefin Hydrosilation (17)

1-octene (150 $\mathrm{mL})$ and $50 \mathrm{mM}$ dicyclopentadienyl platinum (II) chloride in dry chloroform ( $1.00 \mathrm{~mL})$ were placed in a 
3-neck, round-bottom flask equipped with a condenser and a magnetic stirrer. The mixture was heated up to $70^{\circ} \mathrm{C}$ and maintained at this temperature until the solution became clear (at least 1 hour). Then the hydride silica substrate (predried overnight) was added slowly by means of an addition funnel. Once the addition was complete, the solution was heated up to $80-85^{\circ} \mathrm{C}$ and kept at this temperature for 96 hours. The product was washed consecutively with 100-mL portions of toluene (four times), dichloromethane (two times) and diethyl ether (two times). Each washing was followed by about 1 min of vortex stirring and centrifugation. The final product was dried in air at room temperature and then in a vaccum oven at $110{ }^{\circ} \mathrm{C}$ overnight.

\subsubsection{Analytical Procedures}

2.3.2.1. Hydrogen Evolution/Gas Volumetry (18)

Hydride-silica sample (5 to $10 \mathrm{mg}$ ) were carefully weighed in a small vial and about $0.3 \mathrm{~mL}$ of methanol were added in order to remove any trapped air by thoroughly wetting the sample. $\mathrm{HCl}$ solution (5 mM) was then added to fill up the vial. The vial was placed in a recrystalizing dish and about $500 \mathrm{~mL}$ of degassed $5 \mathrm{mM} \mathrm{HCl}$ solution was carefully added to the dish. The vial was covered with a 
special gas collector tube (Figure 1) which had already been filled with HCl solution. Extreme care was taken to avoid the inclusion of any air bubbles during this process. Then a sufficient volume of $10 \mathrm{M} \mathrm{KOH}$ solution was added to make the solution strongly alkaline ( $\mathrm{pH} 12)$. The test was carried out overnight to ensure that the reaction was complete. At the end of this period, the volume of gas was measured along with temperature and barometric pressure. Hydrosilane concentration (mmol $\mathrm{SiH} / \mathrm{g}$ ) was calculated using the ideal gas law.

2.3.2.2. Hydrogen Evolution/Gas Chromatography (19)

Hydrided silica ( 15 to $20 \mathrm{mg}$ ) were weighed in a septumsealed vial. A volume of about $2 \mathrm{~mL}$ of air was withdrawn from the vial by means of a $5-\mathrm{mL}$ gas-tight syringe. About 1 $\mathrm{mL}$ of ethanolic $1 \mathrm{M} \mathrm{KOH}$ solution was carefully injected by using another syringe. The reaction vial was then placed in a heated alumnium block at $60^{\circ} \mathrm{C}$ and the reaction was allowed to proceed for 1 hour, after which the vial was removed from the block and allowed to cool. Ethanol was then injected with a syringe to force the evolved gas into the collection syringe. The syringe valve was closed and the syringe removed from the vial. The gas was then injected to the GC instrument and the hydrogen peak area determined. 


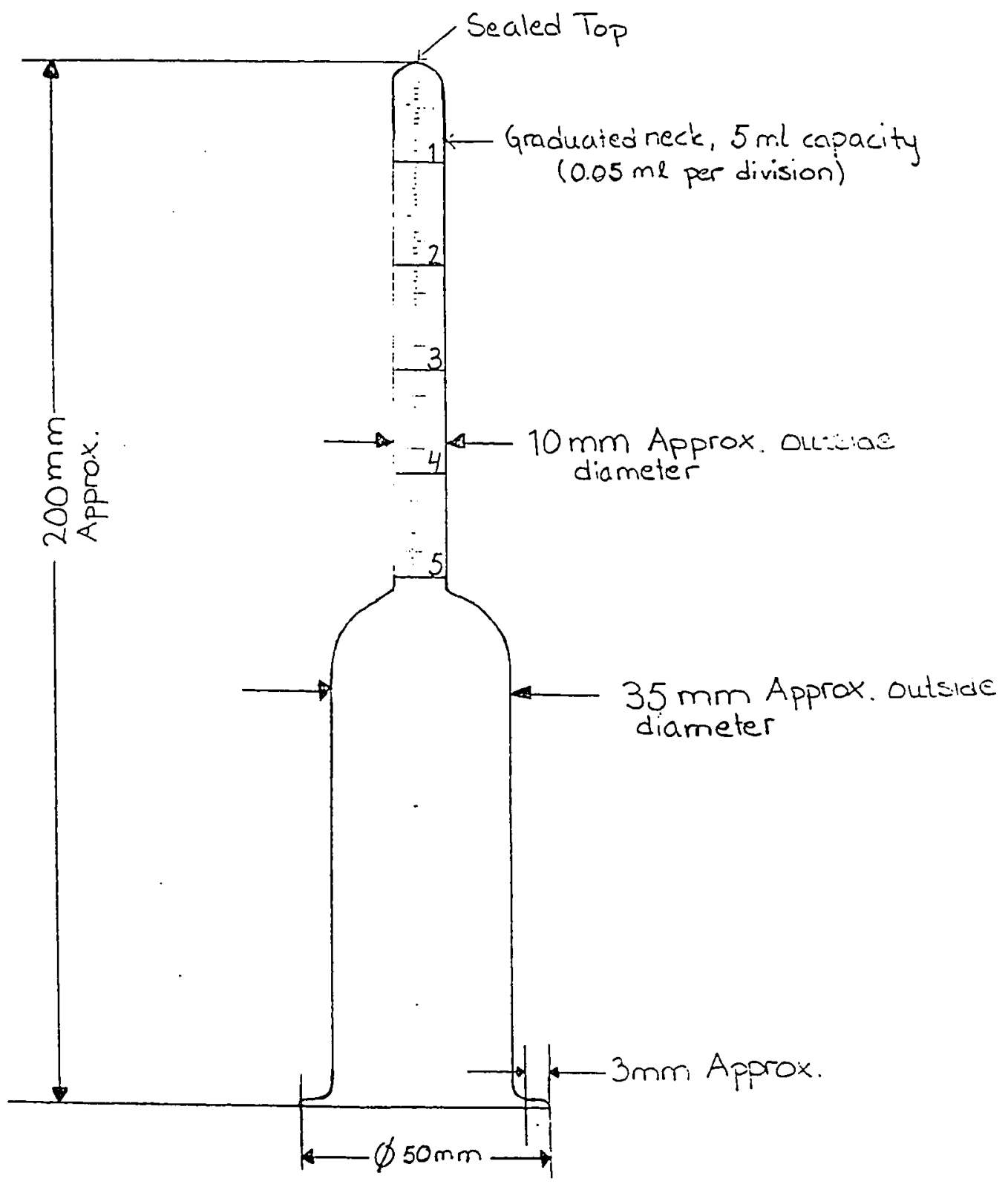

Figure 1. Glass Tube for Hydrogen Gas Collection 
The GC was calibrated against triphenylsilane $\left(\mathrm{Ph}_{3} \mathrm{SiH}\right)$. The latter was standardized by the mercury (II) reduction method described below.

\subsubsection{Mercury Reduction Method (19)}

Triphenylsilane (40 to $50 \mathrm{mg}$ ) were weighed in a stoppered 50-mL conical flask. $\mathrm{Hg}(O A C)_{2}$ solution $(10 \mathrm{~mL}, 8.00 \mathrm{~g}$ $\mathrm{Hg}(\mathrm{OAC})_{2}$ dissolved in $200 \mathrm{~mL}$ of $1: 1 \mathrm{v} / \mathrm{v} \mathrm{CHCl} \mathrm{CH}_{3} / \mathrm{MeOH}$ solution) were added and the mixture stirred occasionally for about 30 min. Methanolic $1.2 \mathrm{M}$ calcium chloride solution (10 mL) was added, followed by 1-2 drops of phenalphthalein indicator. The mixture was finally titrated with methanolic $0.1 \mathrm{M} \mathrm{KoH}$ which was standardized against potassium hydrogen phthalate.

\subsubsection{Long-Term Hydrolysis Test (17)}

Bonded material $(0.560 \mathrm{~g})$ were transferred to a 50-mL centrifuge tube. One $\mathrm{mL}$ of pure dioxane was added and stirred for 5-10 min. Then $30.0 \mathrm{~mL}$ of test solution $(15 \mathrm{mM}$ TFA solution containing $20 \% \mathrm{~V} / \mathrm{v}$ dioxane) were added. Magnetic stirring was carefully adjusted so that a continuous agitation was provided. A 1.5-mL aliquot of well-agitated suspension was taken every 12 hours. The mother suspension was then centrifugated and the test solution was 
renewed in such an amount that the silica/liquid ratio was kept constant. This was done by decreasing the volume of test solution by $1.5 \mathrm{~mL}$. The sample aliquot was transferred to a $12-\mathrm{mL}$ centrifuge tube and centrifugated for $3 \mathrm{~min}$. The solid was washed twice with $3-\mathrm{mL}$ portions of $1: 1 \mathrm{v} / \mathrm{v}$ water/THF, once with THF and once with ethyl ether. The final product was dried at room temperature (until all the ether residue had been evaporated) and then at $110^{\circ} \mathrm{C}$ under vaccum overnight. 


\section{RESULTS AND DISCUSSION}

To form the hydride-modified support, a silane of the general formula $\operatorname{HSix}_{3}$, where $\mathrm{X}$ is an easily hydrolyzable group (e.g., halogen, alkoxy, acyloxy, etc), is reacted with a silica substrate in the presence of water, an acid catalyst and an appropriate solvent. The overall process involves several reactions. Initially, hydrolysis of the three $x$ groups occurs releasing hydrosilanetriol species:

$$
\mathrm{HSiX}_{3}+3 \mathrm{H}_{2} \mathrm{O}------>\mathrm{HSi}(\mathrm{OH})_{3}+3 \mathrm{HX}
$$

This is followed by a series of condensation reactions in which the silanetriol is covalently attached to the silica substrate as well as to other silanetriol(s) via formation of siloxane linkages similar to those existing in the substrate's framework:

$$
\left.\right|_{-\mathrm{Si}-\mathrm{OH}} ^{\mathrm{H}}+\mathrm{HO}-\mathrm{Si}-\ldots+\mathrm{Si-O}-\mathrm{Si}-+\mathrm{H}_{2} \mathrm{O}
$$


It should be noticed that silanol condensation as defined by equation 12 comprises surface attachment of the type

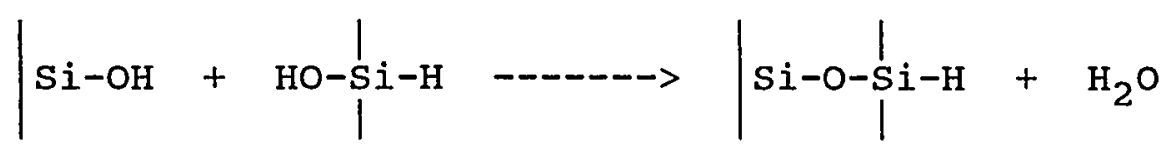

as well as cross-linkings of the type

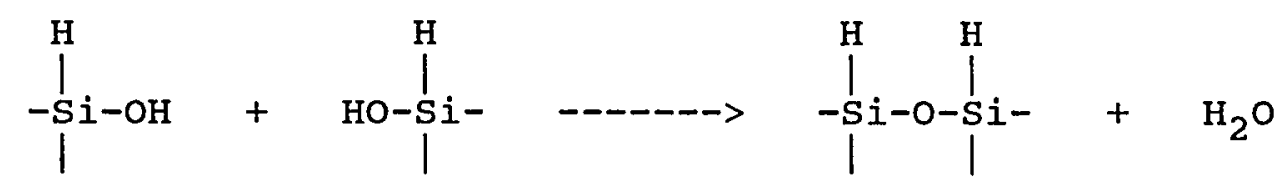

The procedure used can be considered as derived from the socalled "silane coupling" methodology which has been extensively utilized for making surface composites (20). In silane coupling chemistry, organosilanization of a metal oxide substrate is carried out by reacting an organosilane of the formula $\mathrm{RSix}_{3}$ with the inorganic surface:

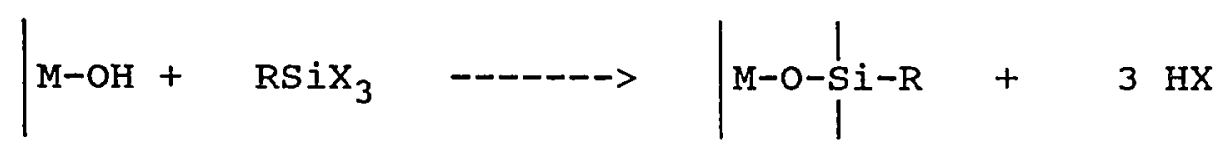

Normally, the R-group contains a terminal reactive functionality which is utilized for ulterior copolymerization with an organic material. The organosilane thus serves as a coupler between the hydrophilic inorganic surface and the organic polymeric material. The utilization of organosilan- 
ization for the preparation of bonded chromatographic phases (equation 2) has prompted extensive studies on the bonding reaction. It has been found that the size of the organic $R-$ group strongly determines the extent at which siloxane linkages (M-O-Si and Si-O-Si) are formed. Steric hindrance from the R-group has been widely accepted as the primary reason for incomplete silanol condensation during the preparation of these type of bonded phases. It seems therefore clear that when the bulky organic moiety is replaced by the smallest possible R-group, that is, hydrogen, silanization should proceed with a maximum of uncondensed silanols. We will refer to this process as "hydrosilanization," to emphasize the replacement of the R-group by hydrogen. In some case the term "deposition" is used. It should be clear from the context that covalent attachment to the substrate (via silanol condensation, equation 12) is involved, as opposed to physical coating.

It should be pointed out that special conditions are required for monolayer deposition to occur. The initial concentration of silane precursor, $\operatorname{HSix}_{3}$, must be sufficiently high for deposition and cross-linking to occur, but must not be so great as to allow bulk polycondensation to proceed to a considerable extent. In the case of a porous substrate such as silica, bulk polymerization of hydrosilanetriol may result in a multilayer deposit and conse- 
quently a significant decrease of the support's pore size, or even to a complete clogging of the porous structure if polycondensation has proceeded too extensively.

similarly with the attachment of coupling silanes on inorganic substrates, experimental conditions such as silane and catalyst concentration, nature of solvent, reaction temperature, etc. should play an important role in the formation of a hydrosilane monolayer on a silica substrate.

\subsection{Preliminary Work}

Because of its wide use in silane coupling procedures (20), ethanol $(95 \% \mathrm{v} / \mathrm{v})$ was used as the reaction medium. In order to explore the general feasibility of the method, some preliminary experiments were carried out using three different concentrations of TES $(0.10 \mathrm{M}, 0.50 \mathrm{M}$, and $0.90 \mathrm{M})$ and three different reaction times (25 minutes, 100 minutes and 20 hours). The deposition of hydrosilane was carried out at room temperature to maintain a minimum ethoxylation of silanol groups

$$
\equiv \mathrm{Si}-\mathrm{OH}+\mathrm{EtOH}-----\longrightarrow>\mathrm{Si}-\mathrm{O}-\mathrm{Et}+\mathrm{H}_{2} \mathrm{O}
$$

Being a competing reaction, extensive ethoxylation might seriously hinder siloxane formation. 
The silicon hydride group in the product is easily identified by a strong infrared absorption in the 2300-2100 $\mathrm{cm}^{-1}$ range. Figure 2 shows partial DRIFT curves for a series of deposition products (100-min reaction time). The Si-H stretching band at 2258-2259 $\mathrm{cm}^{-1}$ agrees very well with that $\left(2260 \mathrm{~cm}^{-1}\right)$ of a hydride silica product prepared by a chlorination/reduction sequence (7). It should be noticed that the Si-H stretching band is consistently accompanied by a shoulder at about $2210 \mathrm{~cm}^{-1}$. This feature suggests that more than one type of $\mathrm{SiH}$ species is present on the silica surface. Besides an obvious increase of total peak area with TES concentration, there does not seem to be a trend in the protuberance of the shoulder. Similar DRIFT profiles are obtained and shown in Figure 3 for another series of deposition products (this time after $25-\mathrm{min}$ of reaction time). Other DRIFT feature of the deposition product is that, in contrast with the product from a chlorination/reduction sequence (7), the SiH bending absorption (a weak band at around $880-885 \mathrm{~cm}^{-1}$ ) is extensively masked by siloxane absorption. A band at $3739 \mathrm{~cm}^{-1}$ due to isolated silanols is also present in the spectra. Figure 4 show typical changes in DRIFT spectra which take place upon hydrosilanization. Several weak bands at 2983, 2938 and 2905 $\mathrm{cm}^{-1}$ due to $\mathrm{C}-\mathrm{H}$ stretching indicate some bonding of ethoxy groups from the ethanol solvent, according to equaltion 14 . 


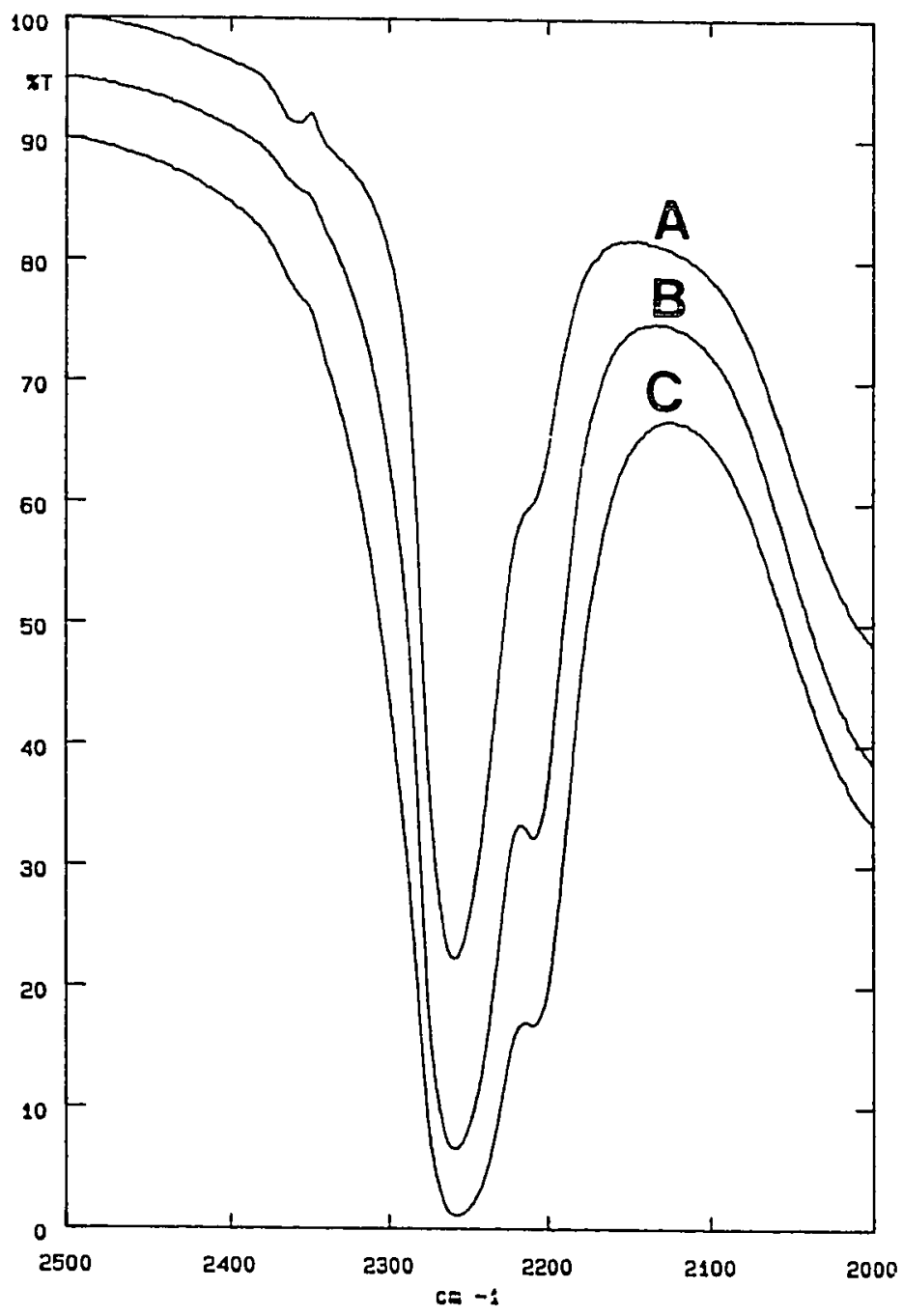

Figure 2. Partial DRIFT Spectra of the products of TES HYdrosilanization of silica: Silica reacted with (A) $0.10 \mathrm{M}$, (B) $0.50 \mathrm{M}$ and (C) $0.90 \mathrm{M} \mathrm{TES}$ in $0.10 \mathrm{M} \mathrm{HCl}$ in $95 \%$ ethanol at room temperature for $100 \mathrm{~min}$. Si-H stretching peaks at (A) $2259 \mathrm{~cm}^{-1}$ with a not well defined shoulder, (B) 2258,2209 $\mathrm{cm}^{-1}$ and (C) $2258,2211 \mathrm{~cm}^{-1}$. 


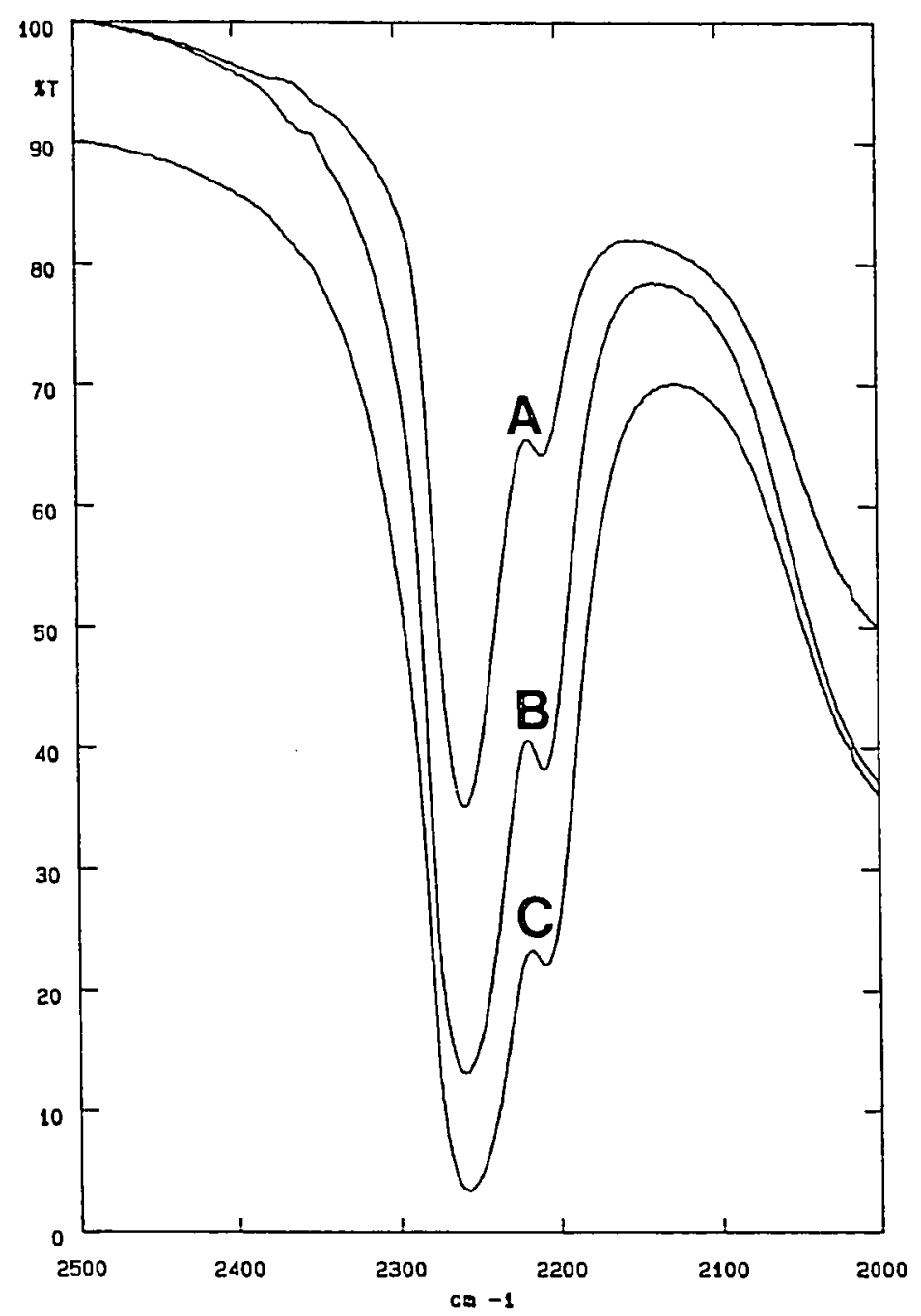

Figure 3. Partial DRIFT spectra of the Products of TES HYdrosilanization of Silica: Silica reacted with (A) $0.10 \mathrm{M}$, (B) $0.50 \mathrm{M}$ and (C) $0.90 \mathrm{M}$ TES in $0.1 \mathrm{M} \mathrm{HCl}$ in $95 \%$ ethanol at room temperature for $25 \mathrm{~min}$. $\mathrm{Si-H}$ stretching peaks at (A) $2258,2209 \mathrm{~cm}^{-1}$ (B) $2258,2209 \mathrm{~cm}^{-1}$, (C) $2257,2209 \mathrm{~cm}^{-1}$. 


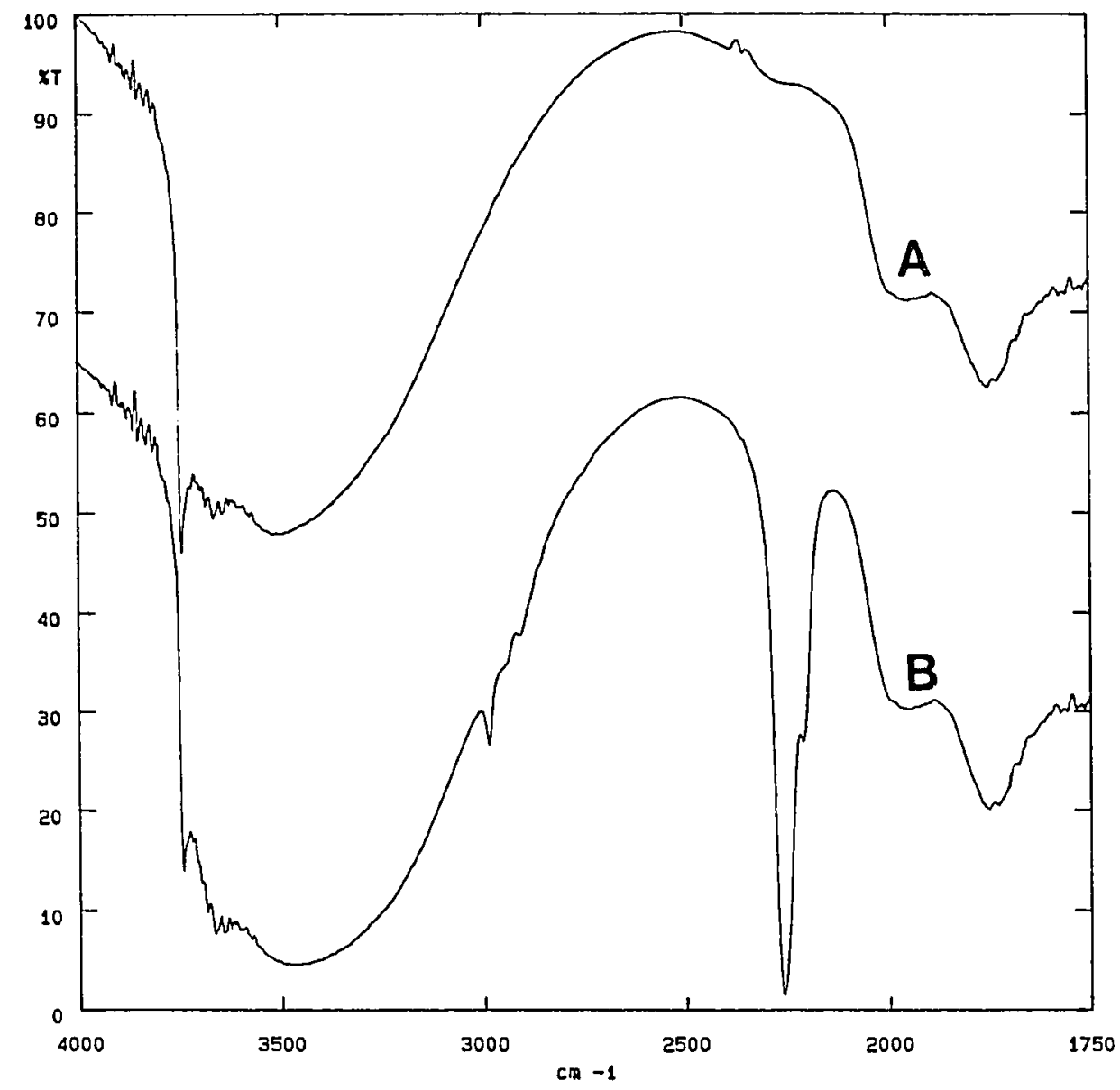

Figure 4. Partial DRIFT Spectra of Native Partisil-40 Silica (curve A) and one of its Hydrosilanization products (curve B). Reaction conditions: $0.50 \mathrm{M}$ TES in $0.10 \mathrm{M} \mathrm{HCl}$ in $95 \% \mathrm{v} / \mathrm{v}$ ethanol; $90 \mathrm{~min}$ reaction time. 
DSC can also be used to have a further insight on the chemical nature of the hydride-modified support. When run in aix, DSC curves show the thermooxidative characteristics of the product, the major process involved being the exothermic oxidation of hydrosilane species to silanols:

$$
\equiv \mathrm{SiH}+1 / 2 \mathrm{O}_{2}-\cdots--->\quad \mathrm{SiOH}+\text { heat }
$$

Figure 5 shows the DSC curves for the same products of Figure 3. It can be seen that for a given reaction time, as TES concentration increases, the DSC oxidation peak becomes bigger, broader, less symmetric and its position shifts to lower temperatures. The same type of observation can be made if a given TES concentration is considered and the reaction time is increased (see Figure 6). Table 2 and Figure 7 summarize the DSC information obtained.

DSC peak broadening strongly suggests an increasing contribution of a second component, probably a $\left(\mathrm{HSiO}_{3 / 2}\right)_{\mathrm{n}}$ polymeric material which might have been formed at the relatively high TES concentration used. In fact, formation of a gel product in which the silica was suspended (overnight experiment with $0.90 \mathrm{M}$ TES concentration) appears to confirm polycondensation of $\mathrm{HSi}(\mathrm{OH})_{3}$. It should be noticed that there was always a stoichiometic excess (molar ratio: 


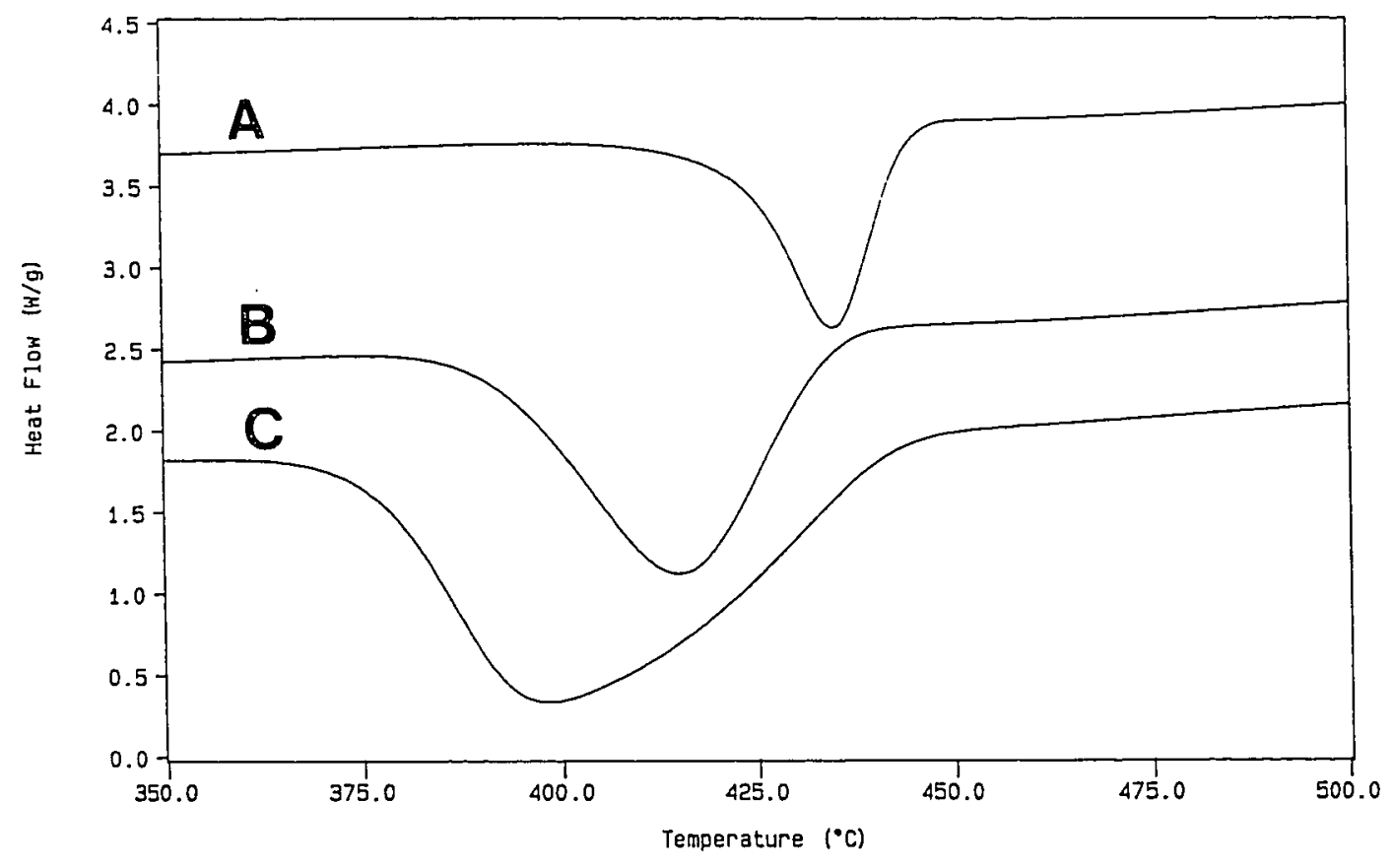

Figure 5. DSC thermograms of the Products of Silica Hydrosilanization with TES: Silica reacted with (A) $0.10 \mathrm{M}$, (B) 0.50 $M$ and (C) $0.90 \mathrm{M}$ TES in $0.10 \mathrm{M} \mathrm{HCl}$ in $95 \%$ ethanol at room temperature for about $25 \mathrm{~min}$. 


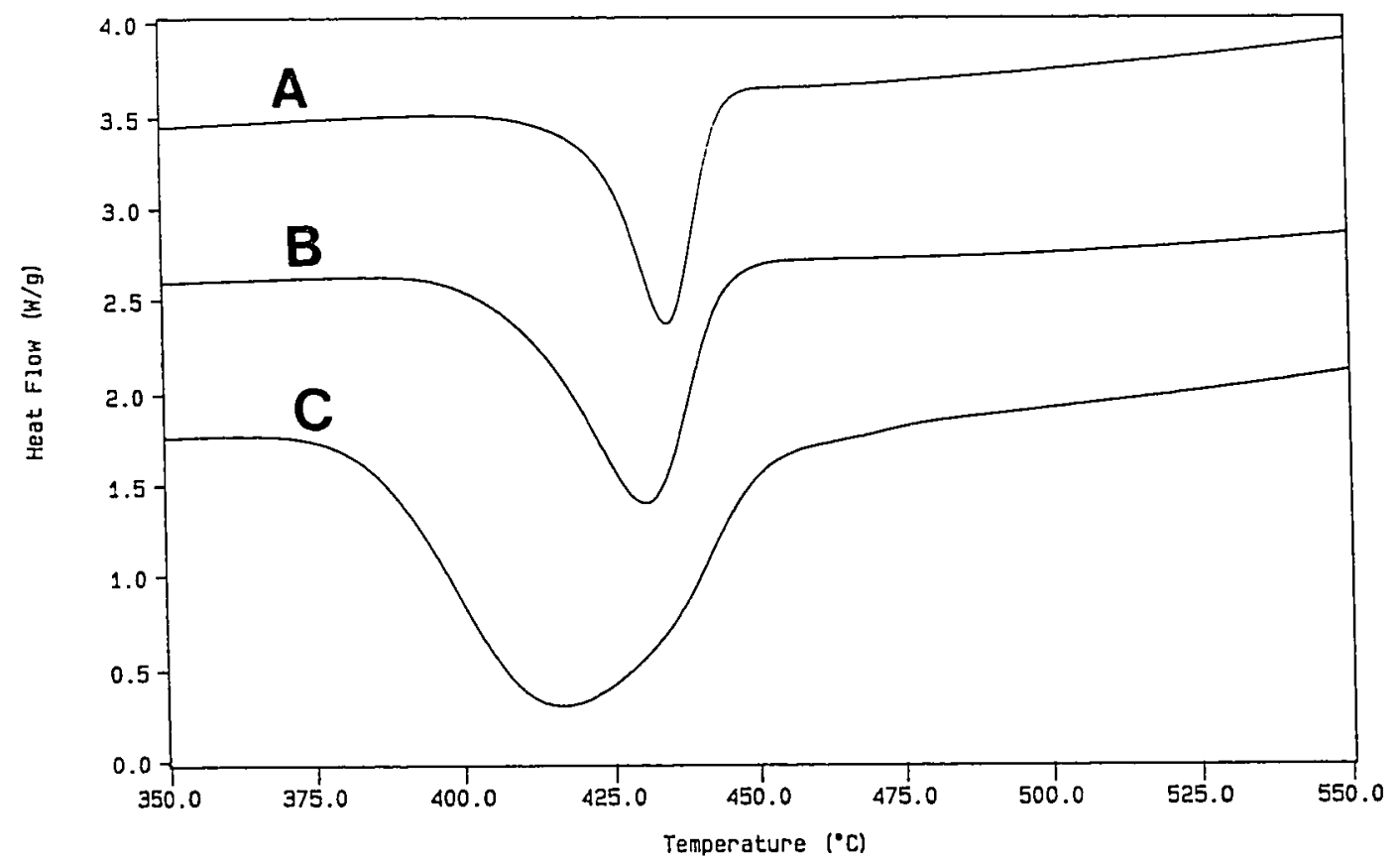

Figure 6. Effect of Reaction Time on DSC Thermograms in Air Silica reacted with $0.10 \mathrm{M}$ TES, $0.10 \mathrm{M} \mathrm{HCl}$ in $95 \%$ ethanol at room temperature for (A) $25 \mathrm{min,} \mathrm{(B)} 100 \mathrm{~min}$ and (C) 20 hours. 


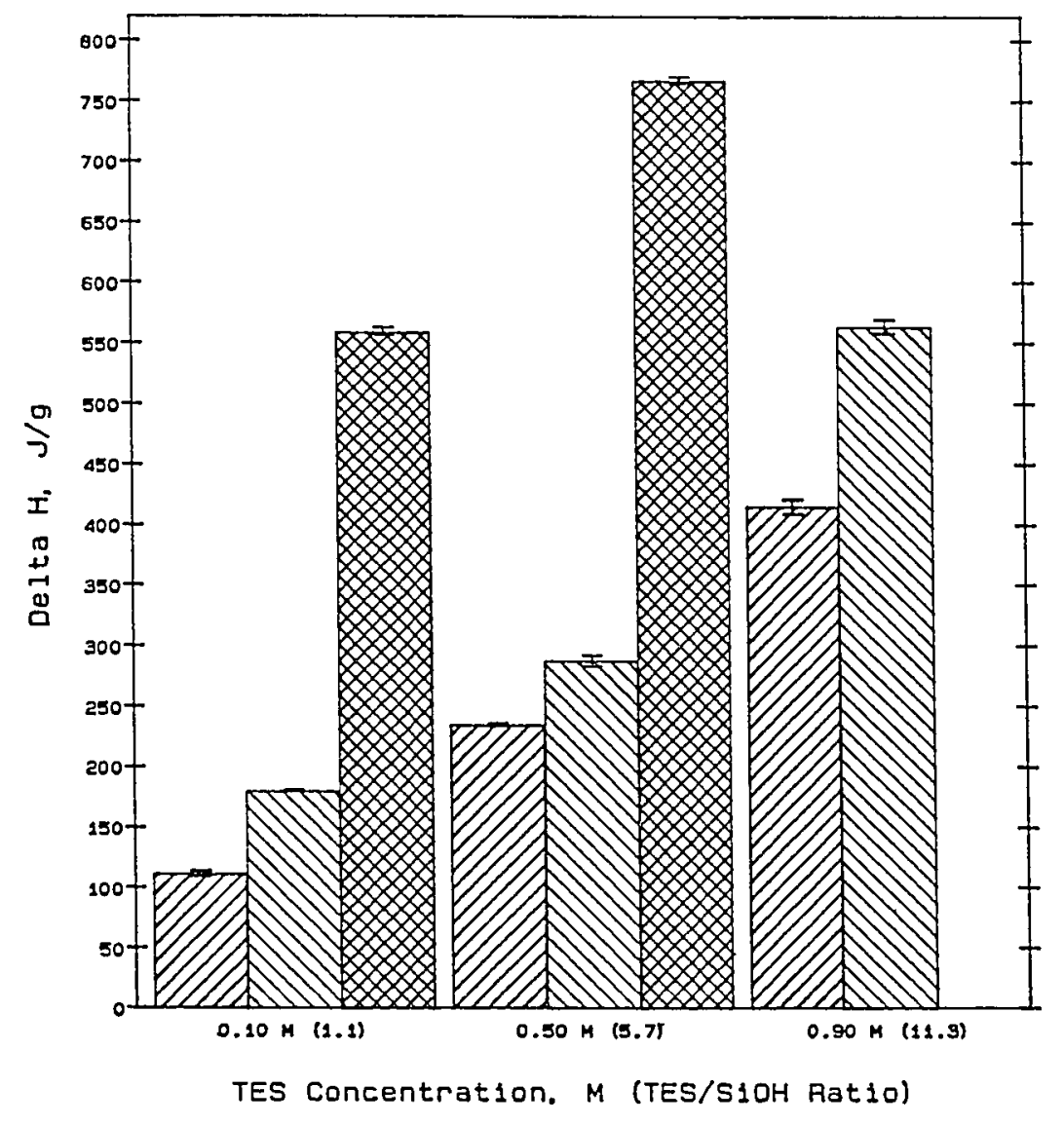

QZIZT 20-25 $\mathrm{min}$

IIII 90-100 min

overnight (20-22 h)

Figure 7. Effect of TES Concentration and Reaction Time on the Oxidation Enthalpy of Hydrosilanized Partisil-40 as Measured by DSC/Air. 
Table 2. DSC/Air Data for Hydrosilanized Partisil-40

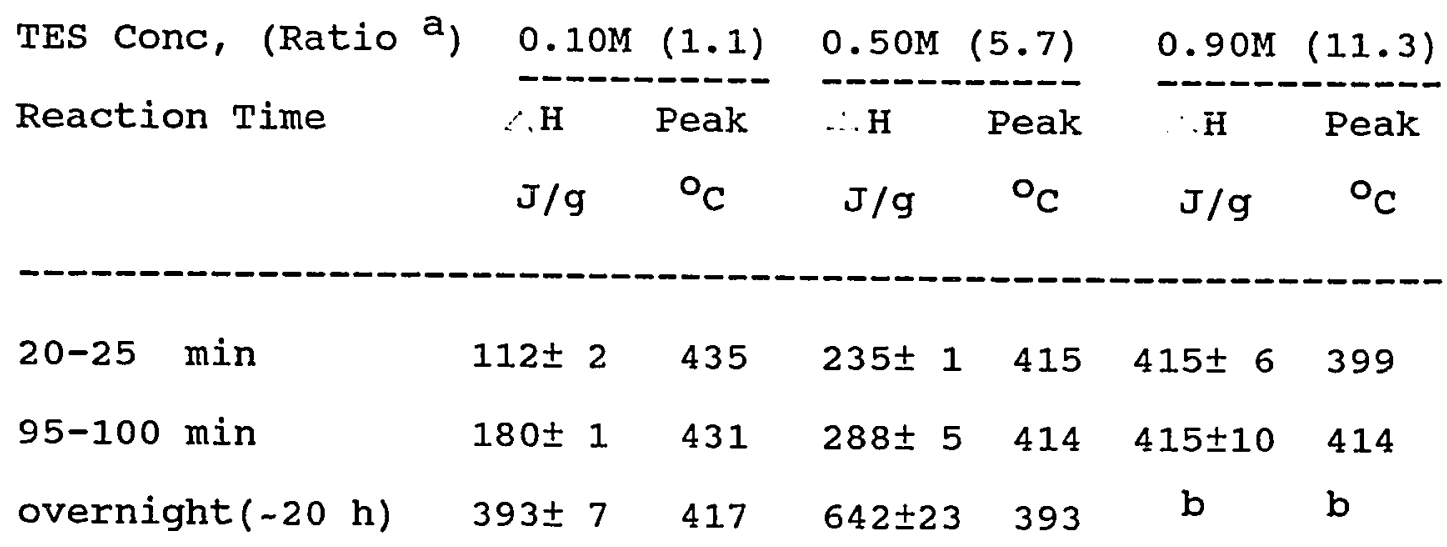

a Molar Ratio: Total TES in solution/Total available silanols from the native silica

b A solidified gel product was formed. No silica product could be recovered.

$1.1,5.7$ and 11.3) of TES, and therefore, multilayer silane condensation was a strong possibility. obviously, increasing the temperature would also have increased this possibility.

The thermooxidative degradation of $\mathrm{SiH}$ species into SioH groups (equation 15 ) is also accompanied by a mass increase which can be easily detected by TGA, as shown in Figure 8 for a series of TES-hydrosilanization products. This figure shows a clear weight increase starting at around $420^{\circ} \mathrm{C}$ and reaching its maximum at around $470^{\circ} \mathrm{C}$ and above. Before and after the oxidative peak, a gradual weight loss 

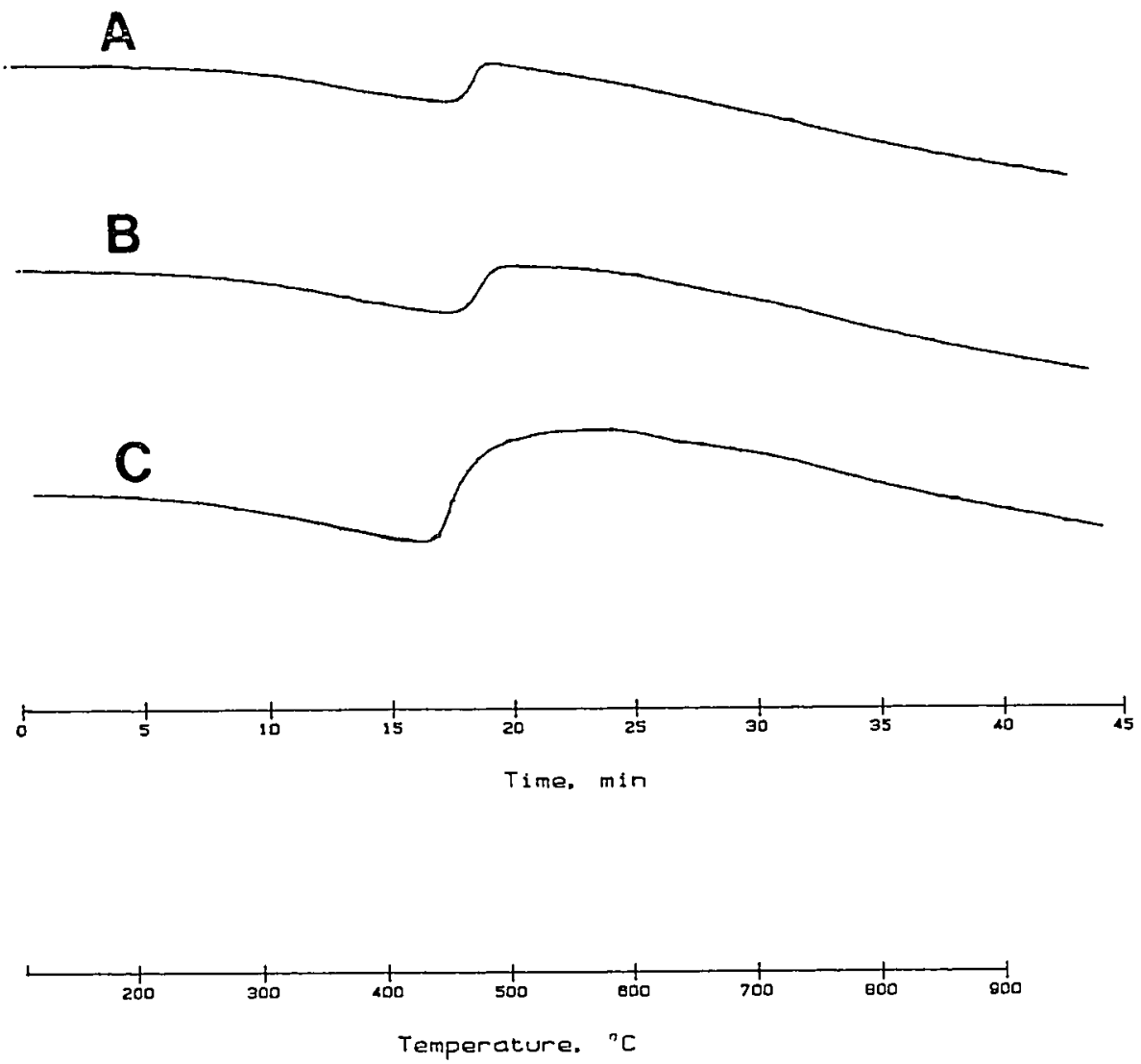

Figure 8. TGA/Air Curves for the Thermooxidation of Hydrosilanized Partisil-40. Curves designated as in Figure 6. 
is observed, as a result of silanol condensation of two adjacent silanols:

$$
2 \equiv \mathrm{Si}-\mathrm{OH} \quad-\cdots---\rightarrow \quad \equiv \mathrm{Si}-\mathrm{O}-\mathrm{Si} \equiv+\mathrm{H}_{2} \mathrm{O}
$$

Similarly with DRIFT and DSC data, the TGA/air peak becomes wider with higher TES concentration and longer reaction time. Also, the temperature at which the oxidation process starts shows a general trend to decrease with increasing TES concentration and reaction time.

When TGA is run under an inert atmosphere (nitrogen or argon), no oxidation peak is present, as expected. However, surprisingly, at high TES concentration and long reaction time, a smooth weight increase is observed above $700^{\circ} \mathrm{C}$, as shown in Figure 9. The effect appears to be most pronounced in the overnight samples (curve c). Further description of this aspect will be addressed in another section.

Qualitatively, DRIFT, DSC and TGA data suggest an increase in SiH coverage when TES concentration and reaction time are increased. Quantitative determination of $\mathrm{SiH}$ carried out by a hydrogen displacement/GC method confirms these observations. This is shown in Figure 10. As expected, the overnight samples show the greatest silane content. It should be pointed out that this is a "bulk" method and therefore it only measures the total silane content inde- 

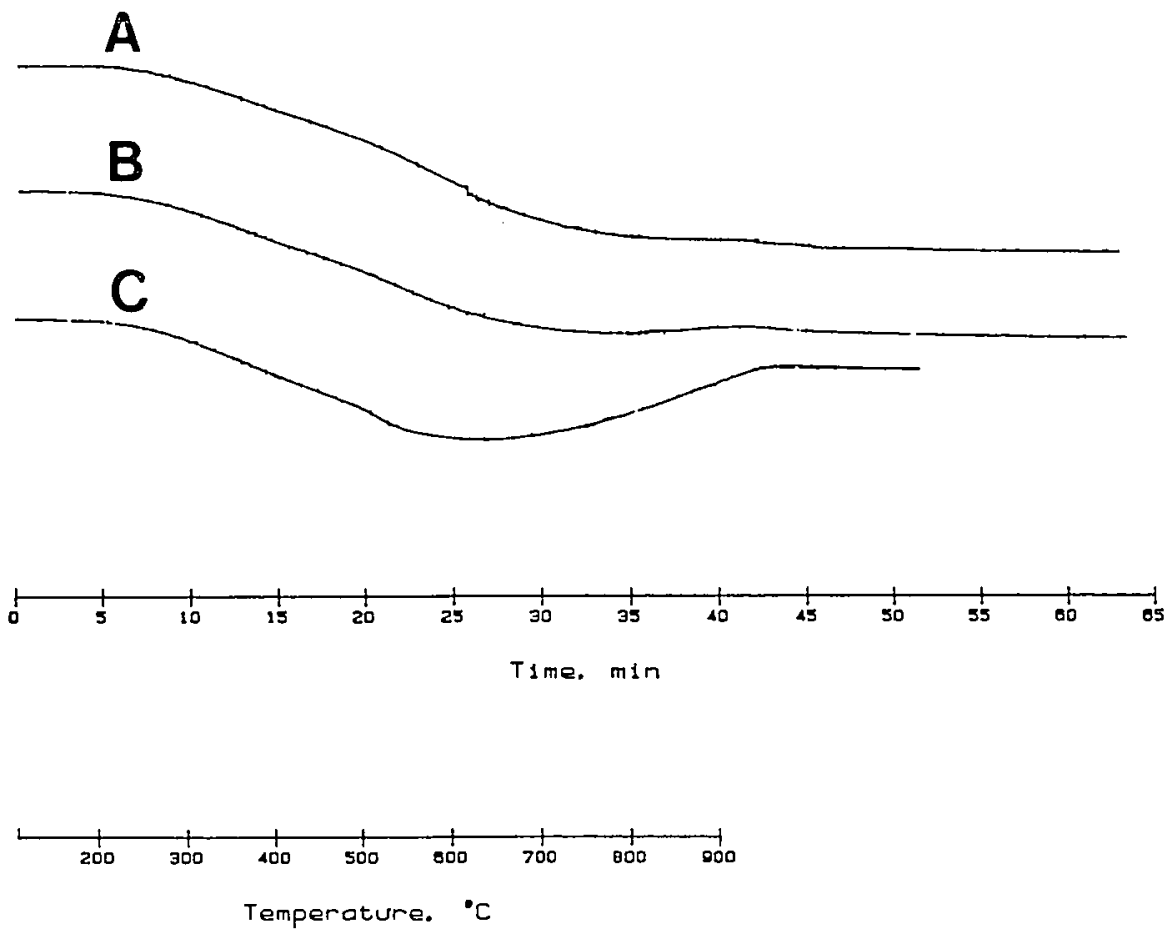

Figure 9. TGA/Nitrogen (or Argon) Curves for Hydrosilanized Partisil-40. Curves designated as in Figure 6. 


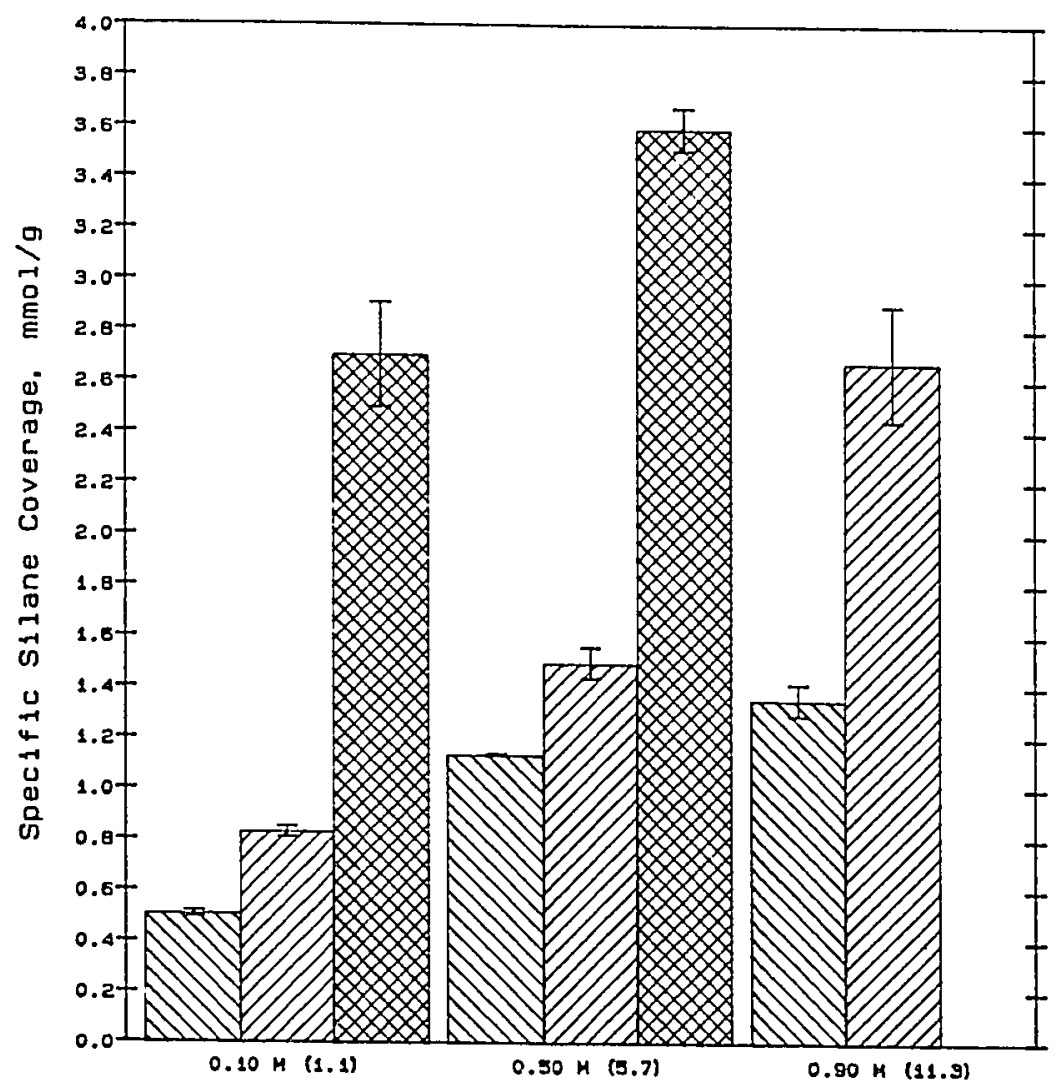

TES Concentration. M (TES/SiOH Ratio)

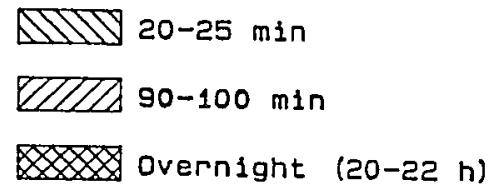

Figure 10. Specific Hydrosilane Coverage as a Function of TES Concentration and Reaction Time. 
pendently from the actual chemical structure of the surface species.

In order to obtain further information on the nature of the polymeric material which might have been formed with certain samples (those associated with high TES concentration and/or long reaction time), an experiment was performed in which no silica substrate was present and the remaining components were reacted under conditions which promoted polycondensation of the hydrosilanetriol hydrolysis product. The mixture ( $0.90 \mathrm{M}$ TES in ethanolic $0.10 \mathrm{M} \mathrm{HCl}$ ) was allowed to react for about 16 hours and the gel product was broken in small pieces, rinsed with solvent (see section 2.3.1.1.1) and allowed to dry until a white solid was obtained. Figure 11 shows the DSc/air curve of the resulting polymeric material with a major peak at $304^{\circ} \mathrm{C}$ along with a broad peak at about $387^{\circ} \mathrm{C}$. It can be seen that these thermooxidative processes occur at temperatures which are lower than those for TES-deposited silicas (Table 2). This observation suggests that broadening and shift of the DSC/air peaks toward lower temperature (Figures 5 and 6) can be attributed to an increasing contribution from polymeric species on the silica surface. As it can be seen in Figure 12, the broad DRIFT absorption from the polymeric $\left(\mathrm{HSiO}_{3 / 2}\right)_{\mathrm{n}}$ product (no silica) strongly suggests a composite peak which may encompass more than one species. 


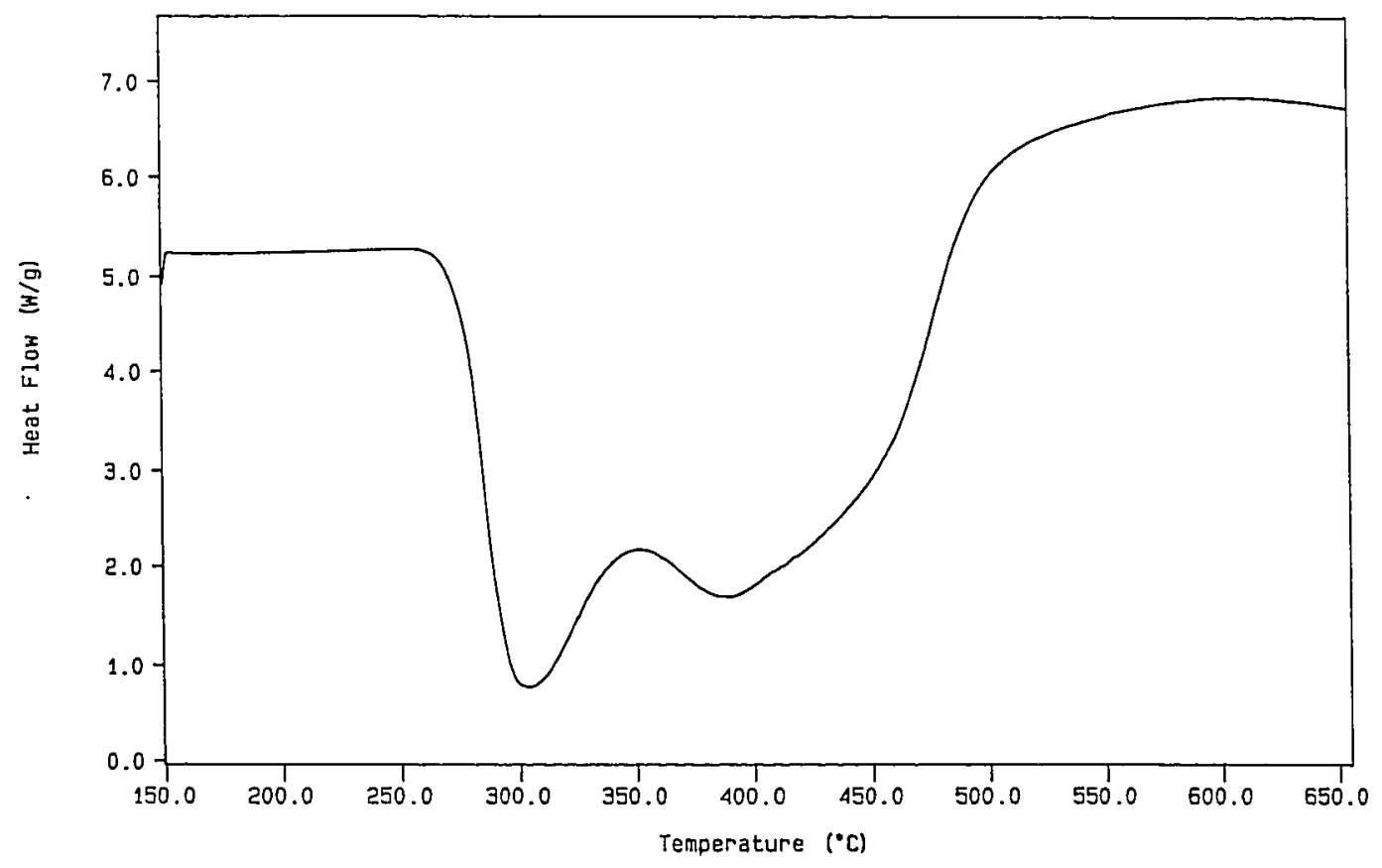

Figure 11. DSC thermograms of the TES Polycondensatic:n Product in the Absence of Silica Substrate: $0.90 \mathrm{M}$ TES reacted with $0.10 \mathrm{M} \mathrm{HCl}$ in $95 \%$ ethanol at room temperature for 16 hours. 


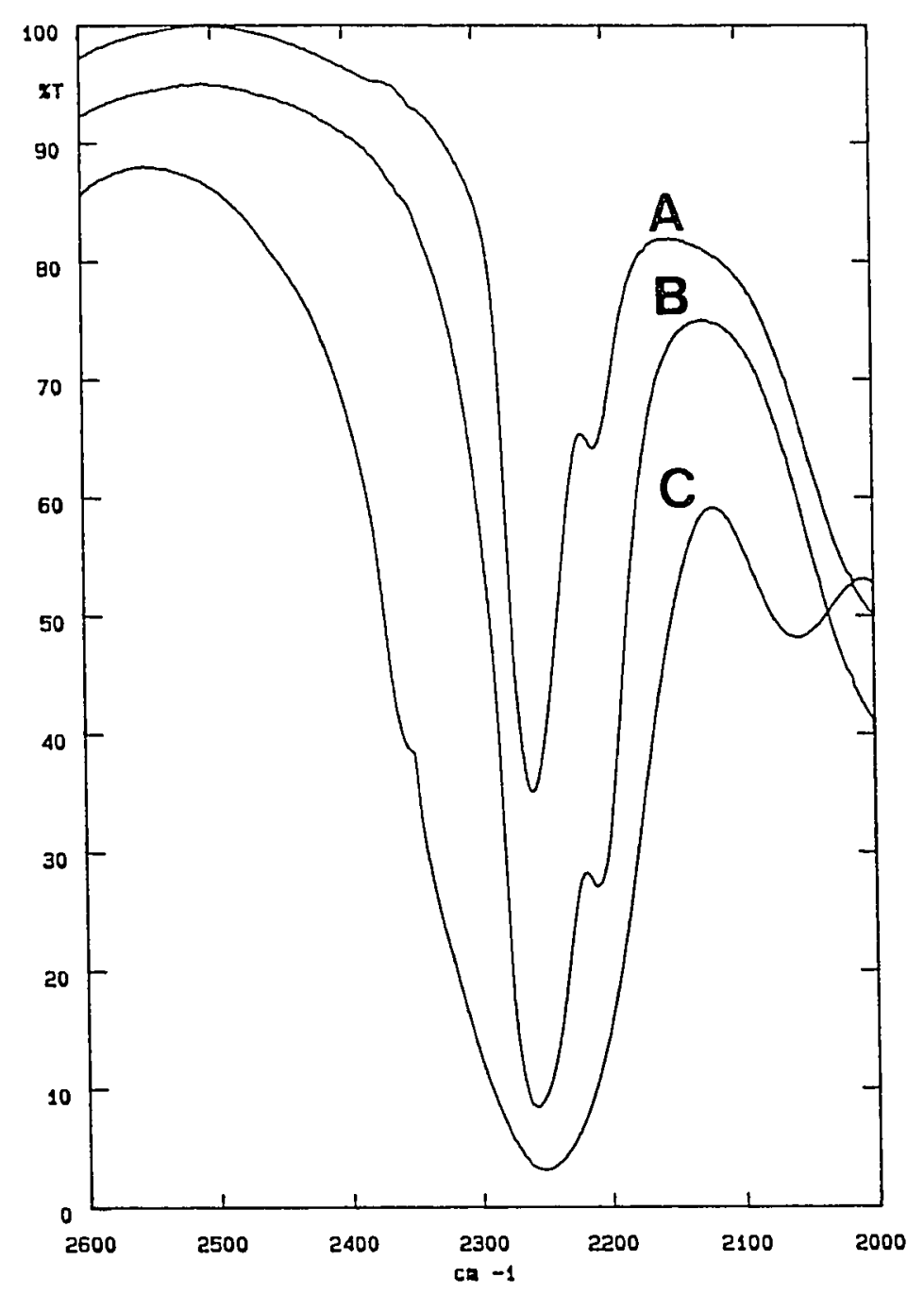

Figure 12. Partial DRIFT Spectra (Si-H Stretching Band) of Hydrosilanization Products: (A) Silica reacted with $0.10 \mathrm{M}$ TES, $0.10 \mathrm{M} \mathrm{HCl}$ in $95 \%$ ethanol at room temperature for 25 min, (B) Silica reacted with $0.90 \mathrm{M}$ TES, $0.10 \mathrm{M} \mathrm{HCl}$ in $95 \%$ ethanol at room temperature for $25 \mathrm{~min}$, (C) $0.90 \mathrm{M}$ TES reacted with $0.10 \mathrm{M} \mathrm{HCl}$ in 95\% ethanol at room temperature for 16 hours (no silica substrate). Peaks at (A) $2258,2209 \mathrm{~cm}^{-1}$, (B) $2257,2209 \mathrm{~cm}^{-1}$, (C) $2253 \mathrm{~cm}^{-1}$. 
In order to prevent the formation of polycondensation products (multilayer $\left.\left(\mathrm{HSiO}_{3 / 2}\right)_{\mathrm{n}}\right)$, the use of lower TES concentration is indicated. We explored the effect of TES concentration in the range for which a total SiH/SioH ratio is around unity; that is, about 40 to $100 \mathrm{mM}$ TES for the silica sample used (Partisil-40). As it can be seen in Figure 13, there is still a shoulder present on the Si-H IR stretching band. Also, there seems to be a trend for this shoulder to decrease at lower TES concentration.

Figure 14 and Table 3 show the thermooxidative characteristics of the same series of samples. In all cases, there is a major oxidation peak at around $435^{\circ} \mathrm{C}$. At the lowest concentration, a well defined second peak at about $408{ }^{\circ} \mathrm{C}$ is also present. Evidence of this peak is also visible in the form of a shoulder in another sample (curve C). Unfortunately, and contrary to DRIFT data (Figure 13) there is no apparent trend in the DSC/air data. This might be due to one or more factors which were not strictly controlled during the experiment; for instance, the rate of addition of TES (used in its neat form) to the silica suspension, the stirring, etc. It can be seen in Table 3 that there was not much variation in the hydrosilane content as indicated by the DSC/air peak area (roughly between 160 and $180 \mathrm{~J} / \mathrm{g})$. This is confirmed by the total siH content of these samples, as determined by a $\mathrm{H}_{2}$ displacement/GC method 


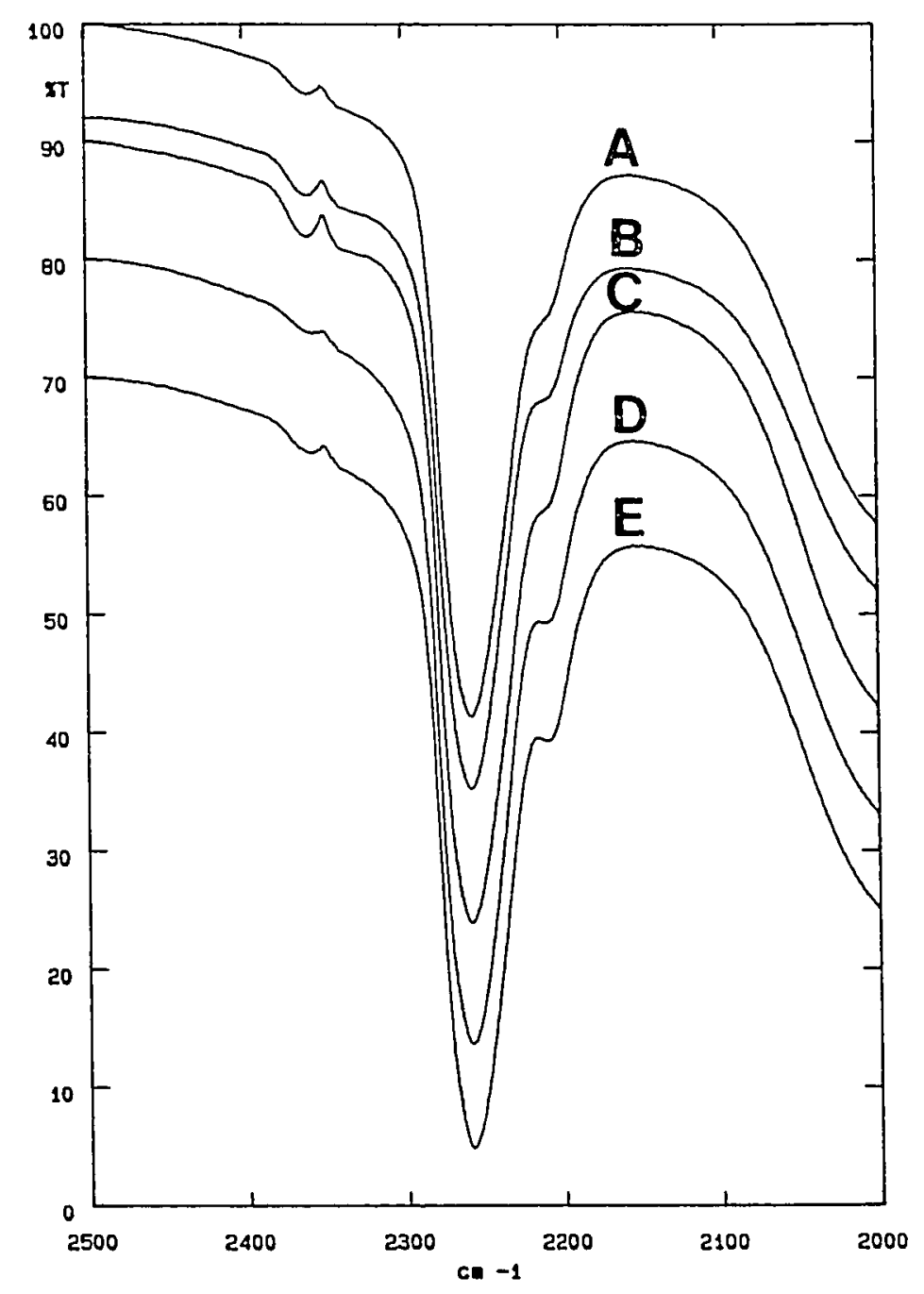

Figure 13. Partial DRIFT spectra (Si-H streching Band) of Hydrosilanized Silica: Silica reacted with (A) $0.045 \mathrm{M}$, (B) $0.060 \mathrm{M}$, (C) $0.075 \mathrm{M}$, (D) $0.090 \mathrm{M}$ and (E) $0.105 \mathrm{M} \mathrm{TES}, 0.10 \mathrm{M}$ $\mathrm{HCl}$ in $95 \%$ ethanol at room temperature for $30 \mathrm{~min}$. Si-H stretching peaks at (A) $2259,2207 \mathrm{~cm}^{-1}$, (B) $2259,2210 \mathrm{~cm}^{-}$ 1 , (C) $2259,2210 \mathrm{~cm}^{-1}$, (D) $2259,2212 \mathrm{~cm}^{-1}$, (E) 2259,2122 $\mathrm{cm}^{-1}$. 


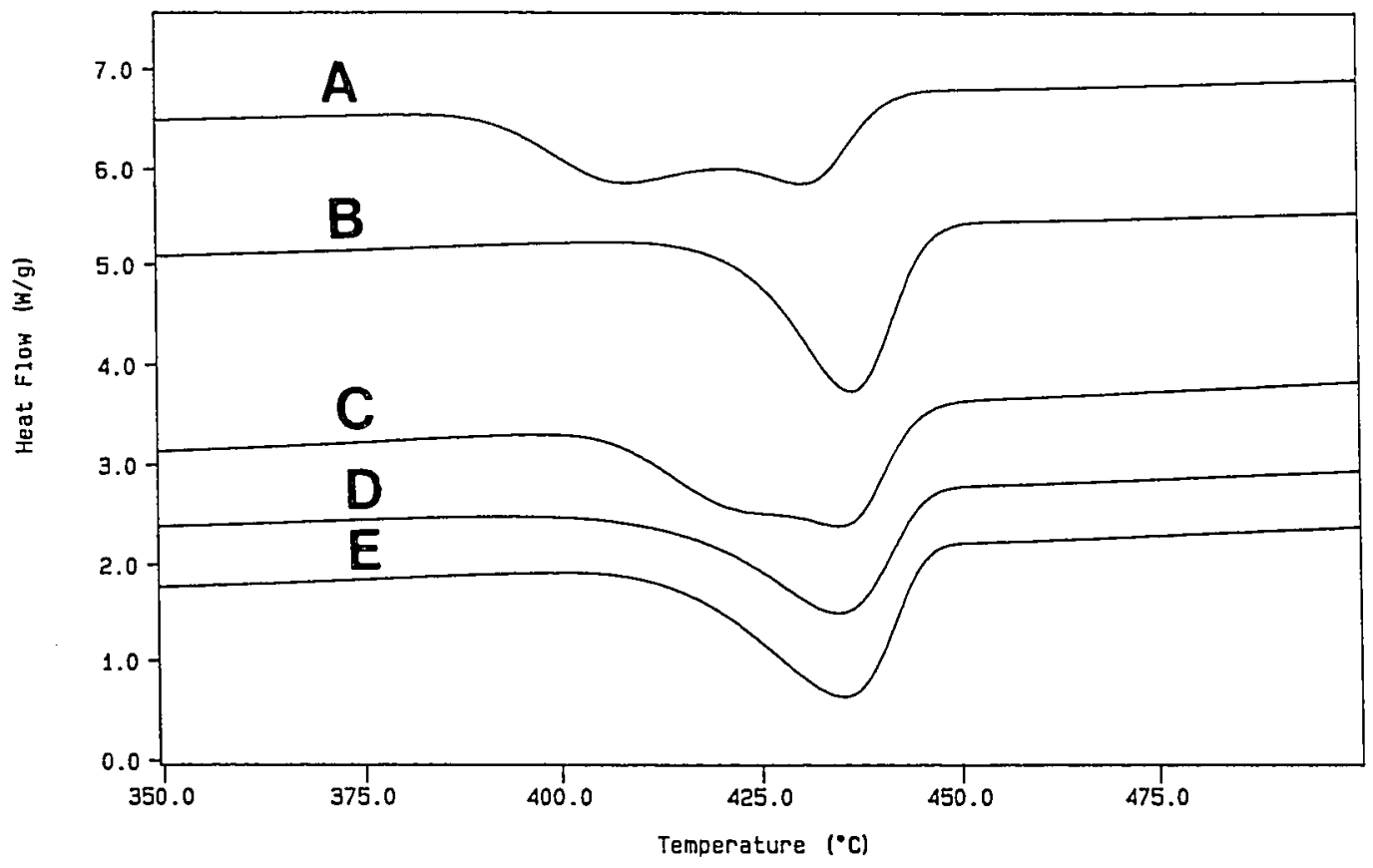

Figure 14. DSC Thermograms of Hydrosilanized Partisil-40. Silica reacted with (A) $45 \mathrm{mM}$, (B) $60 \mathrm{mM}$, (C) $75 \mathrm{mM}$, (D) 90 $\mathrm{mM}$ and (E) $105 \mathrm{mM}$ TES, $0.10 \mathrm{M} \mathrm{HCl}$ in $95 \%$ ethanol at room temperature for $30 \mathrm{~min}$. 
Table 3. Effect of TES Concentration on the Thermooxidation and Hydrosilane Content of Hydrosilanized Silicas (DSC/Air)

\begin{tabular}{|c|c|c|c|c|}
\hline $\begin{array}{l}\text { TES conc. mM } \\
\text { (TES/SioH ratio) }\end{array}$ & $\begin{array}{l}\triangle H \\
J / g\end{array}$ & Pea & ${ }^{c}(s)$ & $\begin{array}{r}\text { Spec. SiH } \\
\mathrm{mmol} / \mathrm{g}\end{array}$ \\
\hline $45(0.50)$ & $184 \pm 1$ & 408 & 431 & 0.38 \\
\hline $60(0.65)$ & $155 \pm 1$ & - & 436 & 0.52 \\
\hline $75(0.80)$ & $183 \pm 1$ & 424 & 435 & 0.44 \\
\hline $90(1.00)$ & $158 \pm 1$ & - & 435 & 0.64 \\
\hline $105(1.15)$ & $181 \pm 1$ & - & 435 & 0.52 \\
\hline
\end{tabular}

(see Figure 15 and last column of Table 3).

with regard to the extent of silane coverage, it should be pointed out that a specific silane coverage of about 0.50 mmol SiH/g would represent a surface silane density of about $1.6 \mu \mathrm{mol} / \mathrm{m}^{2}$ (assuming that no extensive change in $\mathrm{s}_{\mathrm{BET}}$ occurred upon hydrosilanization). The value is inferior to that of the chlorination/reduction product obtained from the same silica substrate (about $2.5 \mu \mathrm{mol} / \mathrm{m}^{2}$, see Table 8 ).

A general implication from these preliminary experiments is that it is possible to obtain a covalently bonded siHlayer on the silica substrate by hydrosilanization with (the hydrolysis product of) TES. Apparently, too high TES concentrations should be avoided to prevent multilayer formation. 


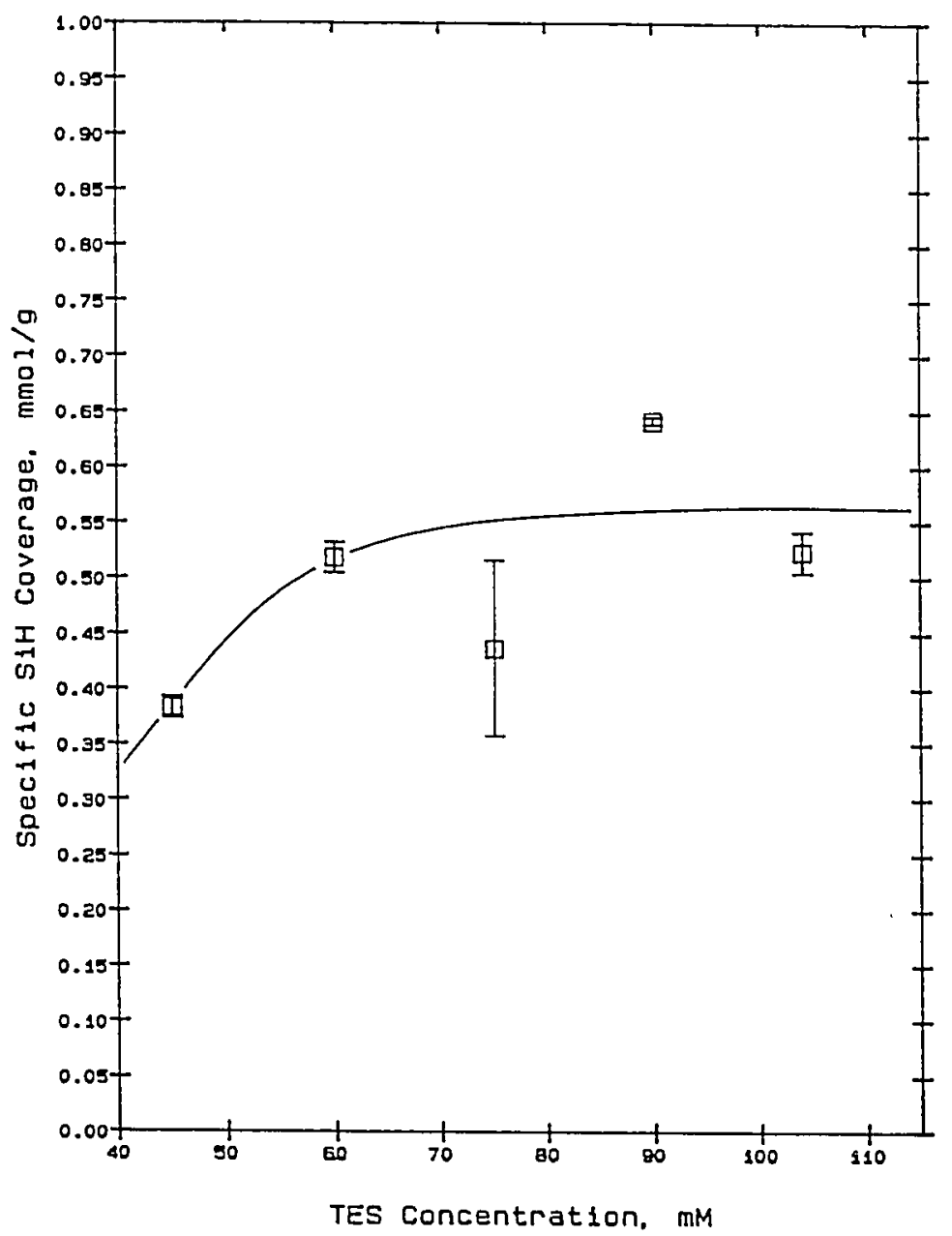

Figure 15. Effect of TES Concentration on SiH Coverage 
Additionally, due to their reaction with silanols (equation 14) the use of alcohol solvents appears to preclude a more efficient elimination (via equation 12) of remaining silanols. The use of an alternate solvent which does not interfere or react with silanols is strongly suggested. An aprotic solvent of the ether type should satisfy this requirement while allowing the use of higher temperatures and thus enhancing silanol condensation (provided it has the proper boiling point).

3.2. Hydrosilanization in an Aprotic Solvent

3.2.1. Solvent and Temperature Effect

It was decided to explore an alternate solvent which does not exhibit the limitations described above. An obvious choice is an aprotic solvent such as an organic ether. Among these, THF (b.p. $67^{\circ} \mathrm{C}$ ) and dioxane (b.p. $101^{\circ} \mathrm{C}$ ) seemed appropriate. Because of its higher boiling point, dioxane seemed to be a better choice.

Unlike the preliminary tests in which neat TES was added to the ethanolic silica suspension, when dioxane was used as a solvent, a solution of TES in dry dioxane was slowly added to the silica/dioxane suspension (see experimental section 2.3.1.1.2.). Figure 16 shows that, in contrast with the 


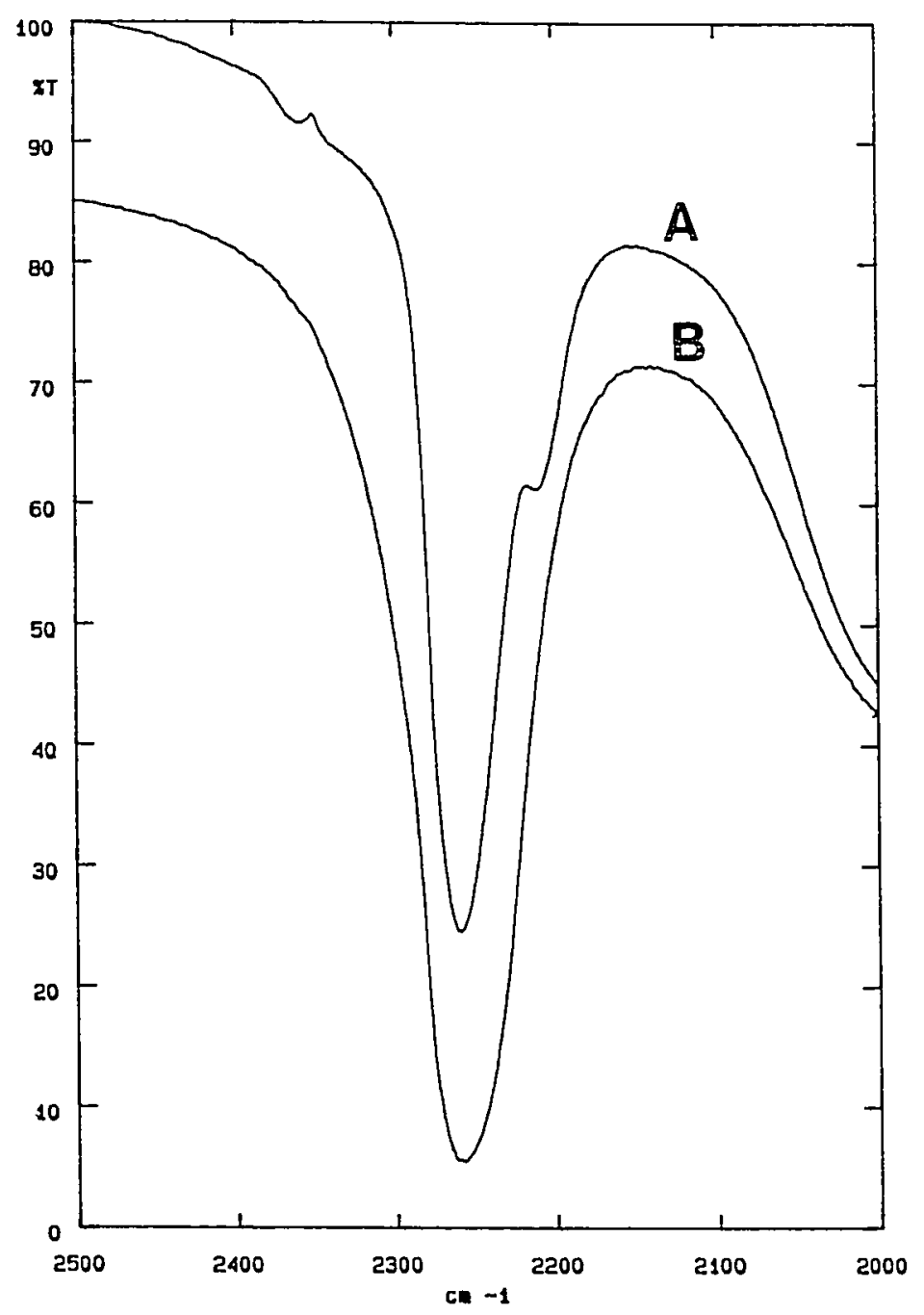

Figure 16. Partial DRIFT Spectra (Si-H stretching Band) of Silica Hydrosilanization Products: (A) Silica reacted with $0.105 \mathrm{M} \mathrm{TES}, 0.10 \mathrm{M} \mathrm{HCl}$ in $95 \%$ ethanol at room temperature for about $30 \mathrm{~min}(\mathrm{TES} / \mathrm{SiOH}=1.1)$, (B) Silica reacted with $0.127 \mathrm{M}$ TES, $0.10 \mathrm{M} \mathrm{HCl}$ in dioxane under reflux for about 60 $\min (\mathrm{TES} / \mathrm{SiOH}=1.2)$. Peaks at (A) $2259,2122 \mathrm{~cm}^{-1}$, (B) 2258 $\mathrm{cm}^{-1}$. 
product prepared with ethanol at room temperature (curve A), a single SiH stretching band is obtained with dioxane at higher temperature, the TES concentration being about the same (curve B). It is also apparent from the figure that a wider and more intense peak is obtained with dioxane, suggesting a greater $\mathrm{SiH}$ coverage. The later observation is confirmed by the DSC data (Figure 17) as well as by the $\mathrm{H}_{2}$ displacement/GC data. This is also shown in Table 4. Since about the same TES concentration was used in both cases, the observed effect can be attributed to a combination of higher temperature and longer reaction time.

It should be noticed that the dioxane-hydrosilanization proauct shows broader DRIFT (Figure 16, curve B) and DSC/air (Figure 17, curve B) bands for the SiH species (SiH stretching at about $2260 \mathrm{~cm}^{-1}$ and $\mathrm{SiH}$ thermooxidation at about $432^{\circ} \mathrm{C}$ ) as compared to the product prepared in ethanol (curve A). Whether these broader peaks were due to more than one SiH species is not possible to extract from the DSC and/or DRIFT information only; solid ${ }^{29} \mathrm{Si}$ NMR could be extremely useful with respect to this structural aspect of the surface SiH species formed upon hydrosilanization.

It should be pointed out that the DRIFT peak area (Table 4) does not accurately reflect the extent of $\mathrm{SiH}$ coverage. This is indicated by an $\mathrm{SiH}$ coverage ratio (dioxane/ethanol) of about 5 and a DRIFT peak area ratio below 2. This is not 


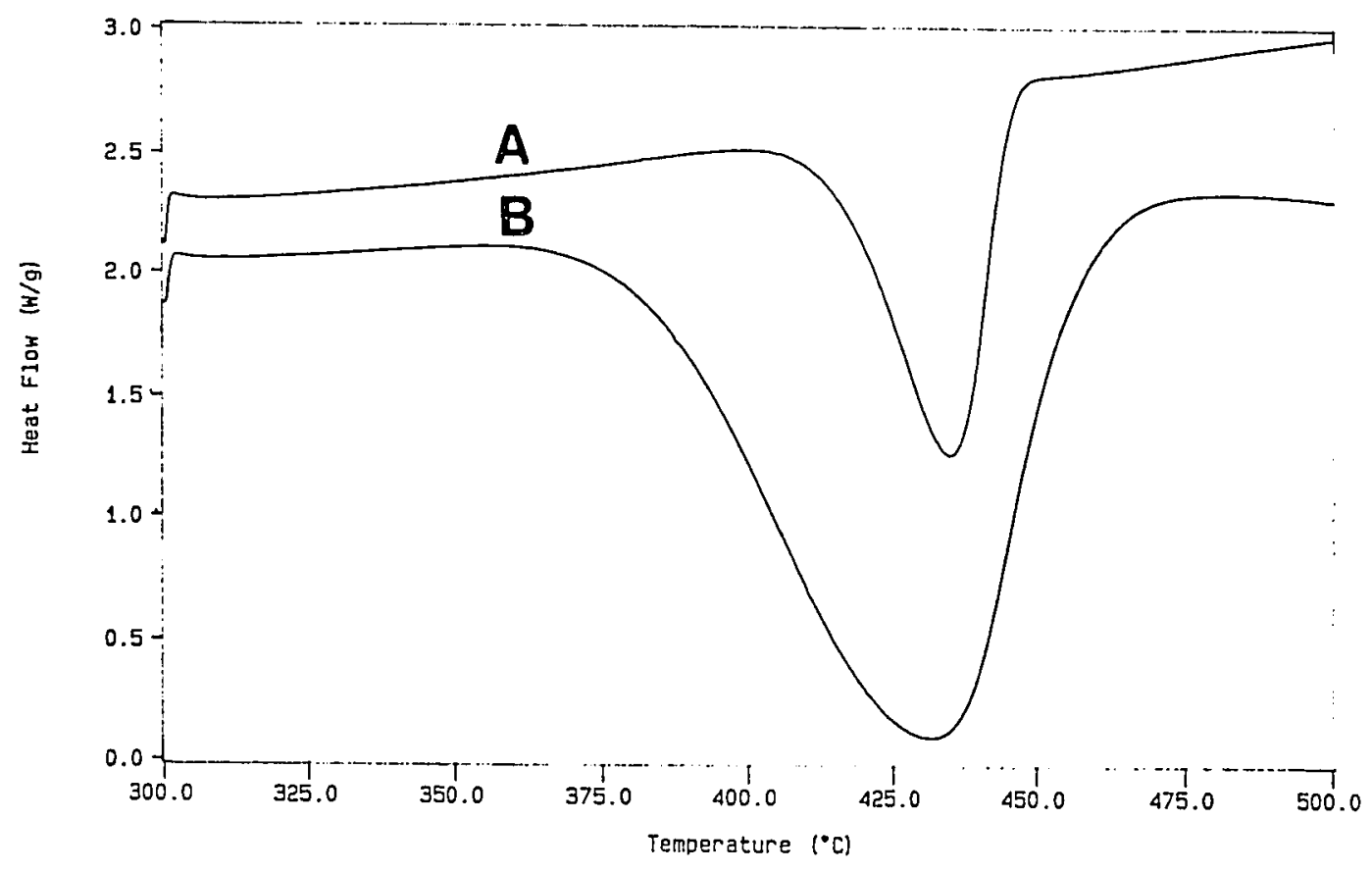

Figure 17. DSC Thermograms of TES on Silica: (A) Silica reacted with $0.105 \mathrm{M} \mathrm{TES}, 0.10 \mathrm{M} \mathrm{HCl}$ in $95 \%$ ethanol at room temperature for $30 \mathrm{~min}(\mathrm{TES} / \mathrm{SiOH}=1.1)$, (B) Silica reacted with $0.127 \mathrm{M}$ TES, $0.10 \mathrm{M} \mathrm{HCl}$ in dioxane under reflux for 60 $\min (\mathrm{TES} / \mathrm{SiOH}=1.2)$. 
Table 4. Effect of Solvent and Reaction Temperature on the TES Hydrosilanization of Partisil-40

\begin{tabular}{cccccc} 
& \multicolumn{2}{c}{ IR } & DSC/Air & SiH Coverage \\
Solvent & Peak(s) & Area $^{\mathrm{a}}$ & $\Delta \mathrm{H}$ & Peak & $\mathrm{mmol} / \mathrm{g}$ \\
& $\mathrm{Cm}^{-1}$ & $\mathrm{~A}^{*} \mathrm{Cm}^{-1}$ & $\mathrm{~J} / \mathrm{g}$ & ${ }^{\circ} \mathrm{C}$ &
\end{tabular}

$\begin{array}{lcccccc}\text { Ethanol } & 2259 & 2122 & 28.8 & 181 \pm 1 & 435 & 0.52 \\ \text { Dioxane } & 2258 & - & 51.9 & 610 \pm 8 & 432 & 2.75\end{array}$

a Integrated between 2350 and $2150 \mathrm{~cm}^{-1}$

surprising, given the limited dynamic range of the infrared technique. Similar $\mathrm{DSC}$ and $\mathrm{H}_{2} / \mathrm{GC}$ ratios do not match very well either ( $\mathrm{AH}$ ratio just above 3 ). Depending on the oxidation peak's shape (particularly when it is "tailing"), the DSC value may or may not accurately measure silane coverage. Since the $\mathrm{H}_{2}$ displacement/GC method does not rely on the measurement of the area of a broad peak such as that obtained by DSC, the $\mathrm{H}_{2} / \mathrm{GC}$ result is more reliable with respect to silane coverage determination. A correlation of the two methods is included in the Appendix.

The most important consequence of changing solvent (and reaction temperature) is that dioxane provided approximately a 5-fold increase in specific silane coverage as evidenced by the $\mathrm{H}_{2}$-displacement/GC method (see Table 4). The superi- 
or hydrosilane coverage obtained with dioxane solvent deserves further comment. The efficiency of hydrosilanization can be readily estimated from the specific $\mathrm{SiH}$ coverage, the specific surface area $\left(\mathrm{S}_{\mathrm{BET}}\right)$, and the surface density of silanols in the starting silica material. Assuming that a monolayer is formed at the TES concentration used $(20 \%$ molar excess), and also that the $s_{B E T}$ value for the hydrided product does not substantially differ from that of the starting silica substrate, a specific coverage of $2.75 \mathrm{mmol}$ SiH/g would correspond to a surface density of about 8.7 $\mu \operatorname{mol} \mathrm{SiH} / \mathrm{m}^{2}$. When the latter value is referenced to the originally available silanols $\left(10.5 \mu \mathrm{mol} / \mathrm{m}^{2}\right.$ for Partisil-40, see Table 7, p. 72), an efficiency of about $84 \%$ is estimated. Although only an estimate, this value not only compares very favorably with that of the ethanolic hydrosilanization product of Table $4(0.52 \mathrm{mmol} \mathrm{SiH} / \mathrm{g}$ correspond to $1.6 \mu \mathrm{mol} \mathrm{SiH} / \mathrm{m}^{2}$, that is about $15 \%$ efficiency) but also with that of a chlorination/reduction product. For the latter, $1.00 \mathrm{mmol} \mathrm{siH} / \mathrm{g}$ corresponds to about $3.2 \mu \mathrm{mol}$ $\mathrm{SiH} / \mathrm{m}^{2}$, i.e., approximately $30 \%$ efficiency.

\subsubsection{Effect of TES Concentration}

Similarly with a previous experiment where ethanol was used as solvent, the effect of TES concentration was ex- 
plored in the vicinity of unity TES/SiOH molar ratio (concentration ranging from 60 to $150 \mathrm{mM})$. This is shown in Table 5 and Figures 18-20. It can be seen that $\mathrm{SiH}$ coverage (Figure 18) increases with TES concentration, and seems to level off at concentration levels for which the TES/SiOH ratio is above unity. The same kind of trend can be seen in Figure 20 for the DSC thermooxidation peak area.

If, again, we assume that there was no substantial change in $\mathrm{S}_{\mathrm{BET}}$ upon hydrosilanization (monolayer formation), the estimated efficiency of hydrosilanization (last column of Table 5) appears to be high under the conditions used.

It should be pointed out that the estimated $\%$ efficiency was inferred from the available silanols on the native silica, as measured by TGA (weight loss between $110^{\circ} \mathrm{C}$ and $900{ }^{\circ} \mathrm{C} ;$ see section 2.2 .2 ). The possibility exists that not all the silanols were condensed at $900{ }^{\circ} \mathrm{C}$ (1) and, therefore, the SiOH content of the native silica may have been underestimated. Additionally, all silanols may not be truly "available" to interact (condense) with hydrosilanetriols, as it is known to happen with "buried" silanols $(1,2,16)$. The implication is that the efficiency values should be used with caution, only for comparison purposes under similar experimental conditions. 


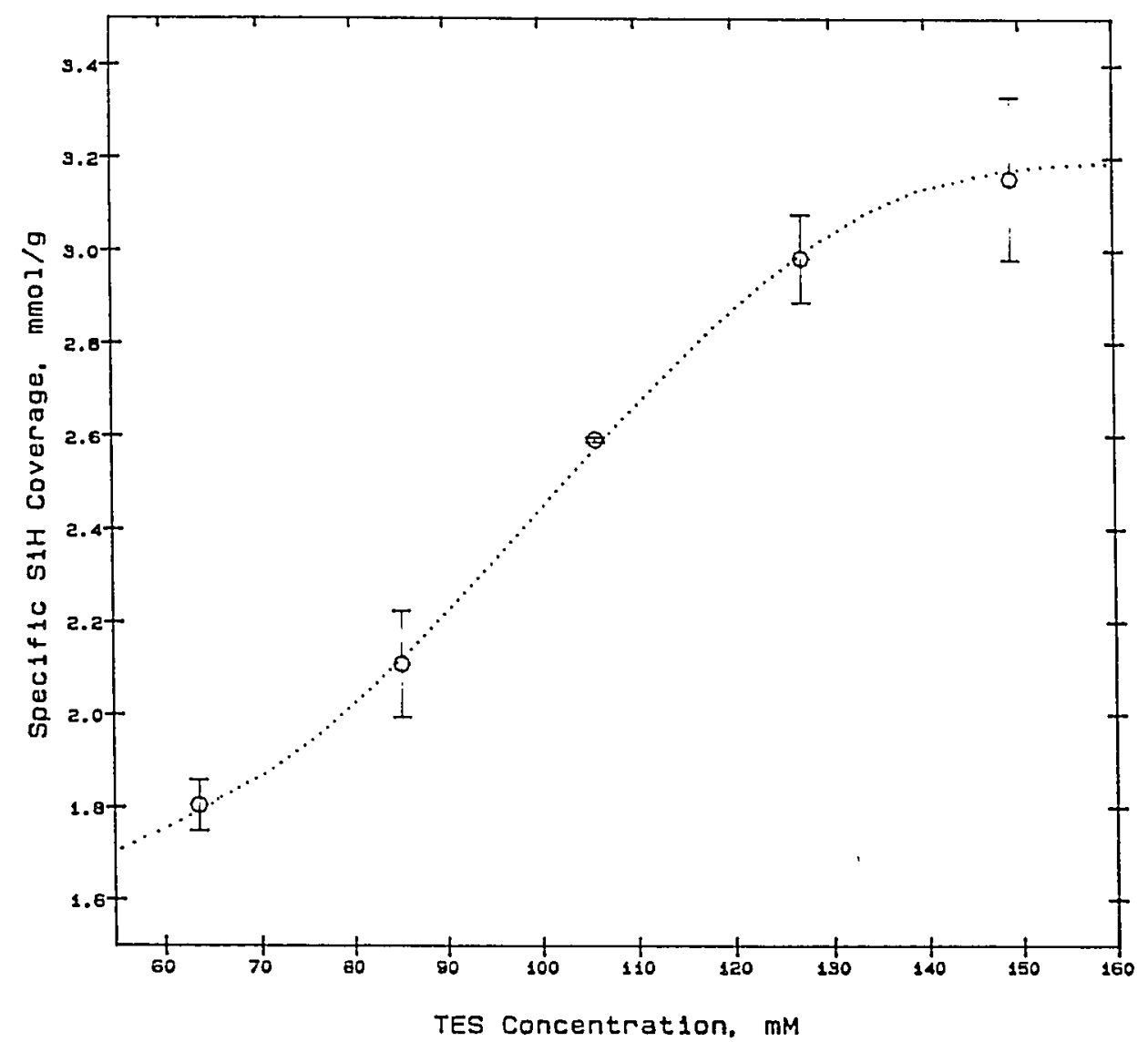

Figure 18. Effect of TES concentration on SiH Coverage, Dioxane Solvent 


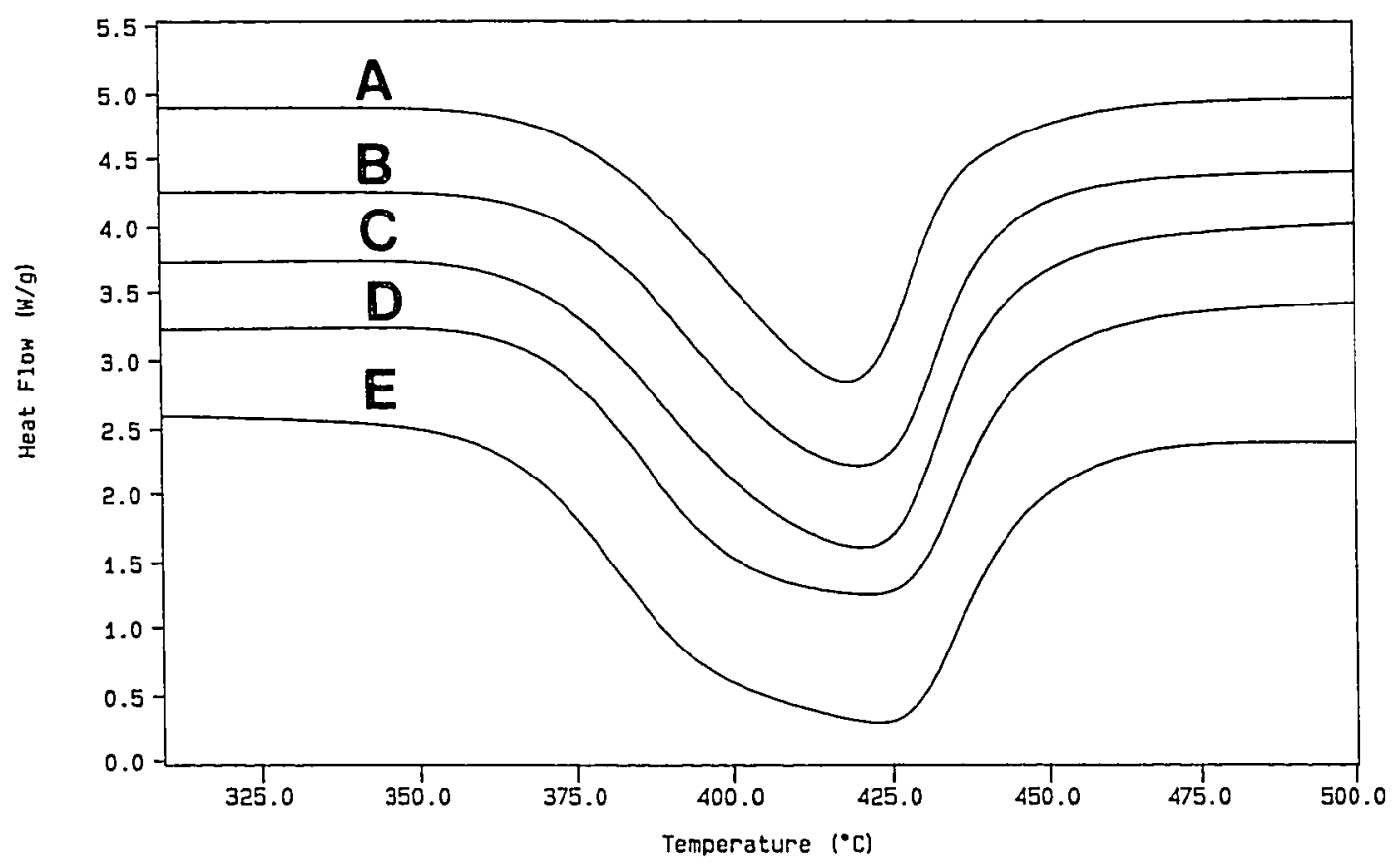

Figure 19. Effect of TES Concentration on the DSC Thermooxidation of Hydrosilanized silica: Silica reacted with (A) 64 $\mathrm{mM}$, (B) $85 \mathrm{mM}$, (C) $106 \mathrm{mM}$, (D) $127 \mathrm{mM}$ and (E) $149 \mathrm{mM}$ TES, $0.10 \mathrm{M} \mathrm{HCl}$ in dioxane under reflux for $60 \mathrm{~min}$. 


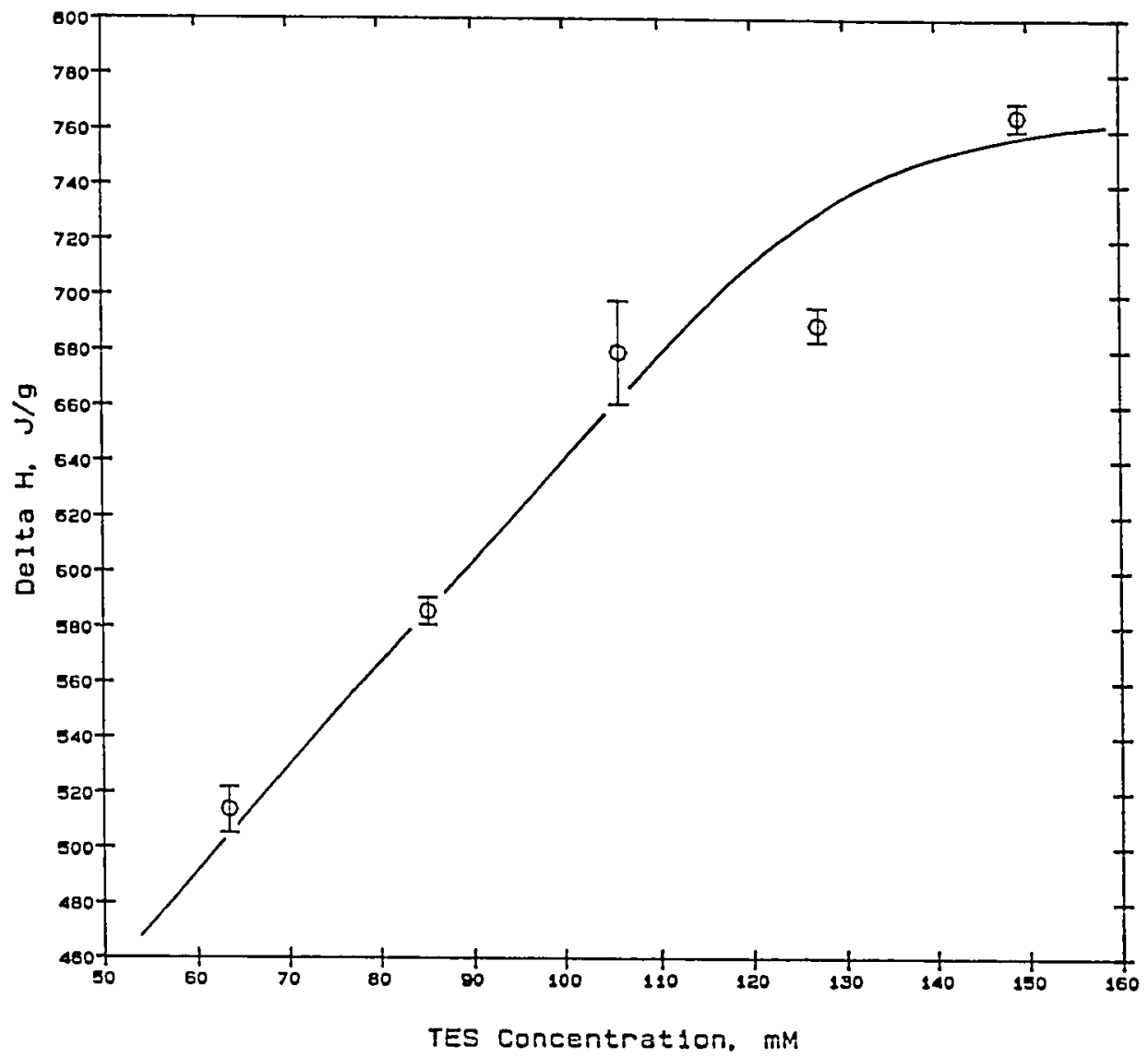

Figure 20. Effect of TES Concentration on the DSC Oxidation Enthalpy, $\Delta H$, Dioxane Solvent. 
Table 5. Effect of TES Concentration on Silica Hydrosilanization

\begin{tabular}{|c|c|c|c|c|c|}
\hline \multirow{3}{*}{$\begin{array}{c}\text { TES Conc } \\
\mathrm{mM}\end{array}$} & \multirow{3}{*}{$\begin{array}{l}\text { siH/sioH } \\
\text { molar ratio }\end{array}$} & \multicolumn{2}{|c|}{ DSC/Air } & \multicolumn{2}{|l|}{ Spec. SiH } \\
\hline & & ------ & ---- & Coverage & Estimated ${ }^{a}$ \\
\hline & & $\hat{\Delta} \mathrm{H}, \mathrm{J} / \mathrm{g}$ & Peak, ${ }^{\circ} \mathrm{C}$ & mmol/g & \%ffficiency \\
\hline 63.5 & 0.60 & $514 \pm 8$ & 418 & $1.81 \pm 0.05$ & 60 \\
\hline 85.2 & 0.80 & $586 \pm 5$ & 420 & $2.11 \pm 0.10$ & 71 \\
\hline 105.8 & 1.00 & $679 \pm 19$ & 420 & $2.59 \pm 0.02$ & 89 \\
\hline 127.1 & 1.20 & $689 \pm 6$ & 421 & $2.98 \pm 0.10$ & 104 \\
\hline 149.0 & 1.40 & $765 \pm 5$ & 423 & $3.16 \pm 0.15$ & 111 \\
\hline
\end{tabular}

a Referenced to the available silanols in the starting material Partisil-40 (see Table 7,P.71)

3.2.3. Effect of Reaction Time

In order to investigate the rate at which $\mathrm{HSi}(\mathrm{OH})_{3}$ is chemisorbed on the silica surface, a small sample aliquot was removed periodically (over a period of 24 hours) from the reaction mixture. The results are shown in Table 6 and Figures 21 and 22. The data indicate a fast surface chemisorption as evidenced by the fact that the hydrosilane coverage curve (Figure 21) rapidly attained a constant value 


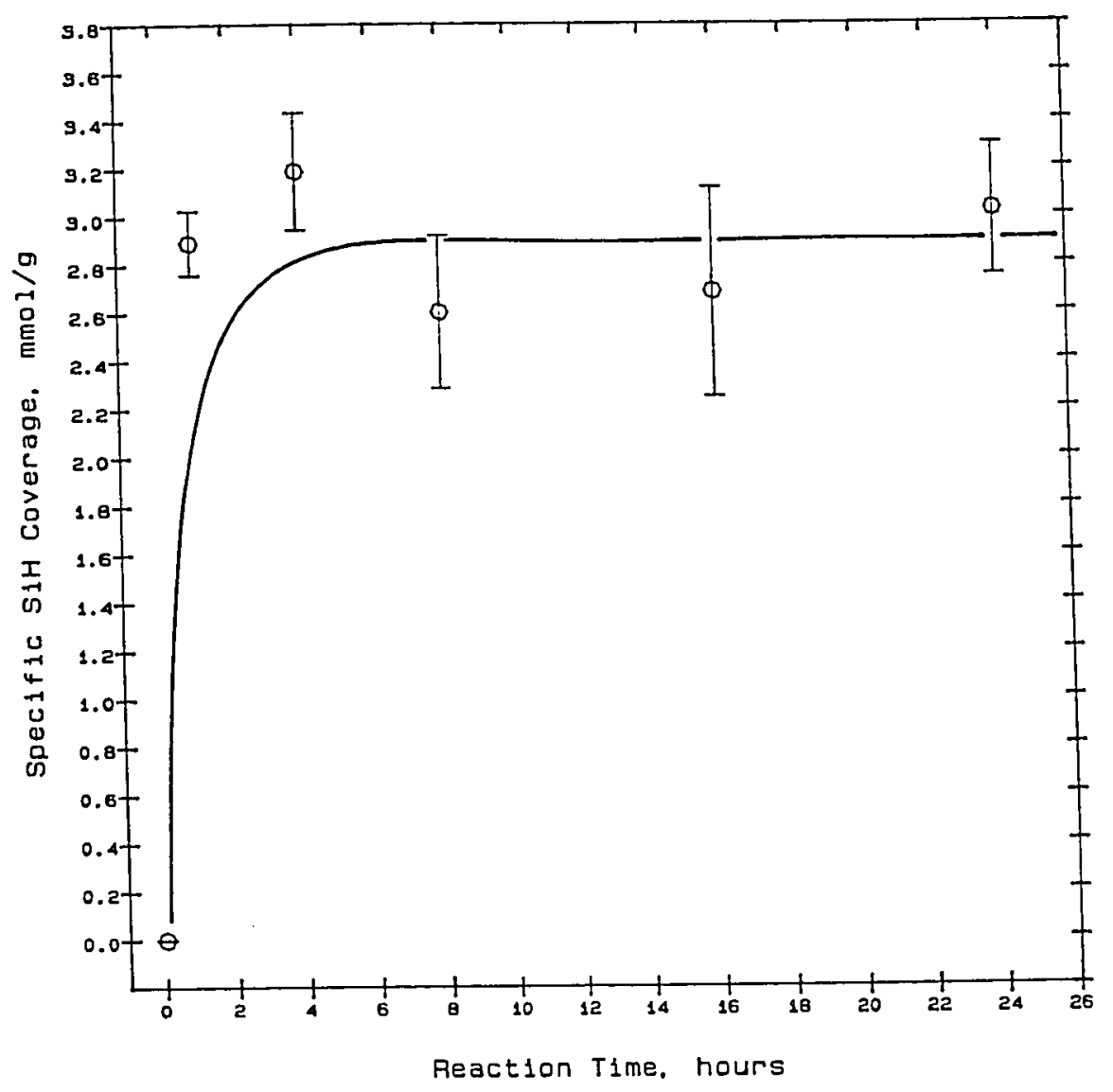

Figure 21. Effect of Reaction Time on SiH Coverage: Silica reacted with a $20 \%$ TES molax excess of TES in dioxane containing $0.10 \mathrm{M} \mathrm{HCl}$. 


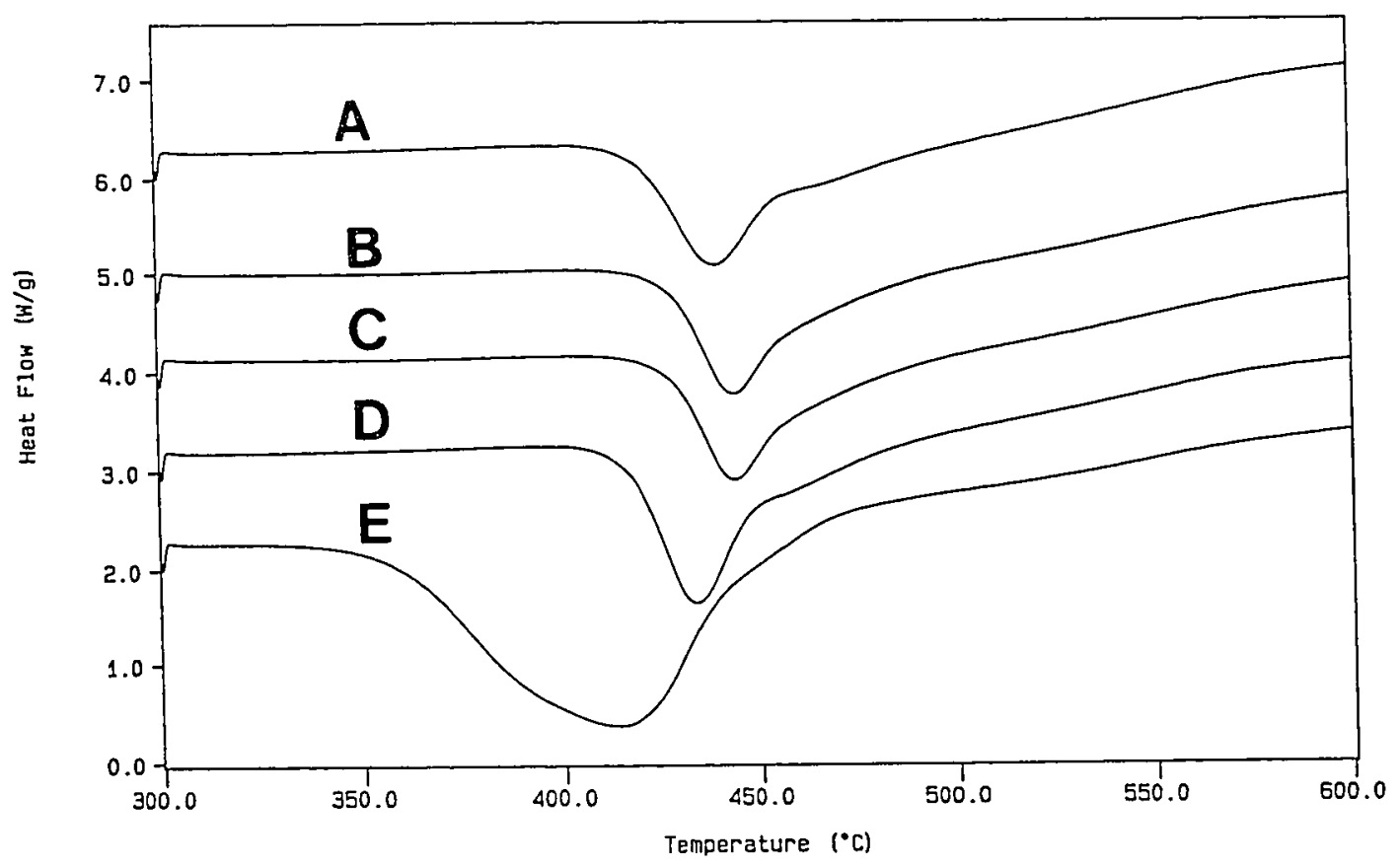

Figure 22. Effect of Reaction Time on DSC Thermograms: Silica reacted with $0.127 \mathrm{M}$ TES, $0.10 \mathrm{M} \mathrm{HCl}$ in dioxane under reflux for (A) 1 hour, (B) 4 hours, (C) 8 hours, (D) 16 hours, (E) 24 hours. 
Table 6. Effect of Reaction Time on Hydrosilanization with TES Carried out in Dioxane Solvent

\begin{tabular}{|c|c|c|c|c|}
\hline Time & \multicolumn{2}{|c|}{ DCS/Air } & Spec. & SiH Coverage \\
\hline $\mathrm{h}$ & $\Delta \mathrm{H}, \mathrm{J} / \mathrm{g}$ & Peak, ${ }^{\circ} \mathrm{C}$ & & $\mathrm{mmol} / \mathrm{g}$ \\
\hline 1 & $456 \pm 8$ & 440 & & $2.9 \pm 0.1$ \\
\hline 4 & $470 \pm 6$ & 452 & & $3.2 \pm 0.2$ \\
\hline 8 & $392 \pm 5$ & 444 & & $2.6 \pm 0.3$ \\
\hline 16 & $481 \pm 5$ & 434 & & $2.7 \pm 0.4$ \\
\hline 24 & $757 \pm 5$ & 414 & & $3.0 \pm 0.3$ \\
\hline
\end{tabular}

of about $3.0 \mathrm{mmol} / \mathrm{g}$ which, as started before, indicates a highly efficient coverage. The obvious implication is that a significant reduction of the preparation time for the hydride intermediate is possible through the use of this approach. A contact (reaction) period of about 1 hour was used in subsequent experiments.

3.2.4. Effect of Acid Catalyst

It is a well-known fact that the nature and concentration of the acid catalyst strongly affects the polycondensation as well as the redissolution of silicic acids and its derivatives $(13,16)$. The effect of the concentration of the 
acid catalyst used, $\mathrm{HCl}$, on the extent of hydrosilane chemisorption was examined next. Kurennaya and Slinyakova (13) have shown that the polycondensation of triethoxysilane (which produces a polyhydrosiloxane gel) speeds up with increasing amounts of the acid catalyst (they used $\mathrm{HCl}$ and $\left.\mathrm{H}_{3} \mathrm{PO}_{4}\right)$. Very short gelling times were observed for $\mathrm{HCl}$ concentrations of $1 \mathrm{M}$ and above. Since we are interested in avoiding gel formation, lower concentrations were used in our case to promote monolayer deposition (1 to $100 \mathrm{mM} \mathrm{HCl}$ range). Figure 23 shows the variation of the extent of hydrosilane chemisorption with the concentration of $\mathrm{HCl}$ catalyst. As expected, the extent of silane deposition increases with acid concentration. At a concentration of about $0.10 \mathrm{M}$ the effect seems to level off. Thus, under the conditions used $(20 \%$ molar excess of TES and 1 hour reaction time), a concentration of about $0.10 \mathrm{M} \mathrm{HCl}$ should be used to produce a satisfactory hydrosilane coverage.

DRIFT spectra (Figure 24) further confirms the presence of hydrosilane on the silica surface at all three HCl concentrations. Figure 25 depicts the thermooxidation behavior of the products as a function of $\mathrm{HCl}$ concentration. Surprisingly, the product resulting from the lowest $\mathrm{HCl}$ concentration (1.0 mM) shows virtually no thermooxidation peak at the temperature range under consideration. This observation does not mean that no silane was deposited, since DRIFT 


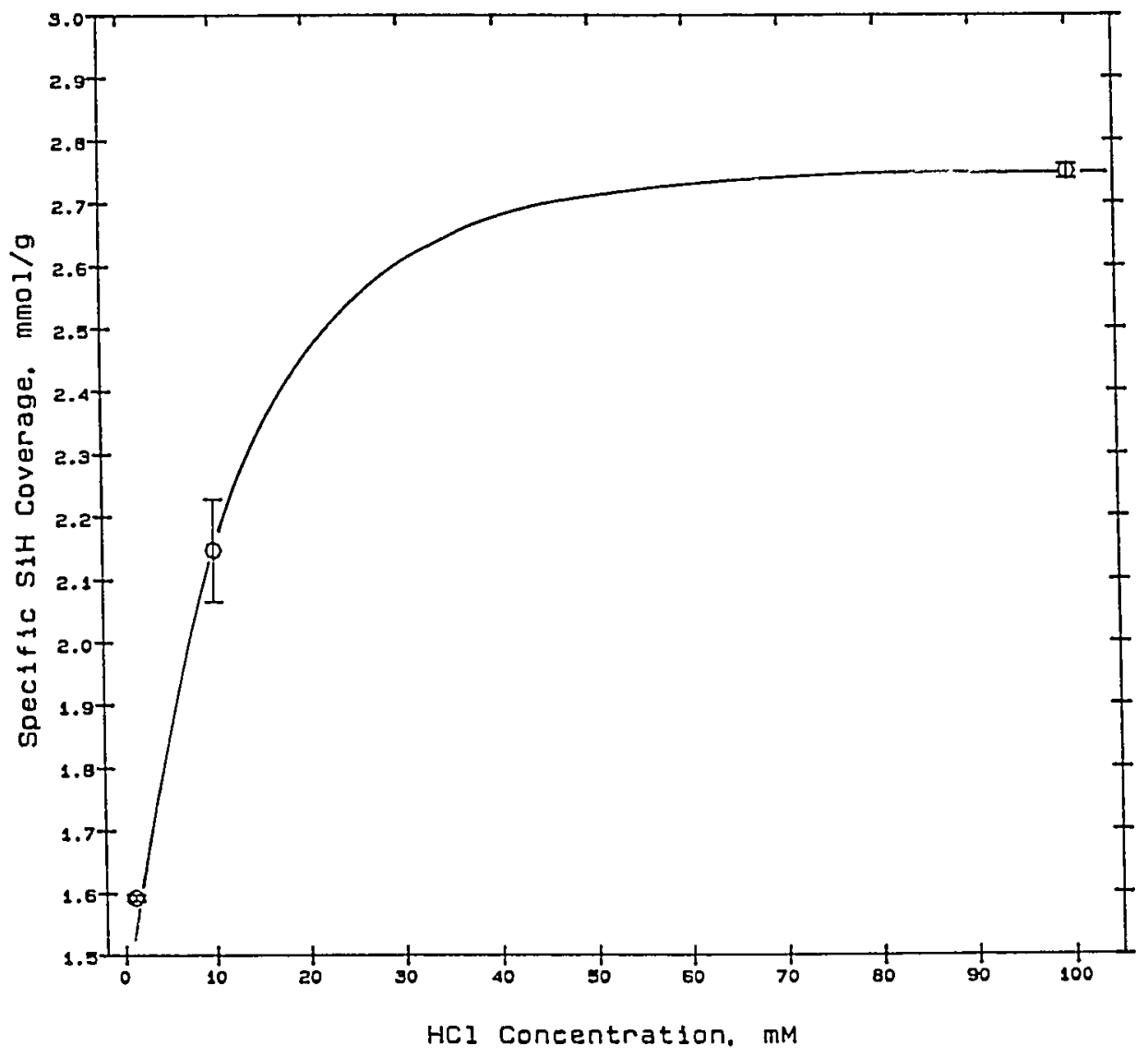

Figure 23. Effect of $\mathrm{HCl}$ Concentration on Hydrosilane Coverage: Silica reacted with a $20 \%$ TES molar excess under reflux for 1 hour. 


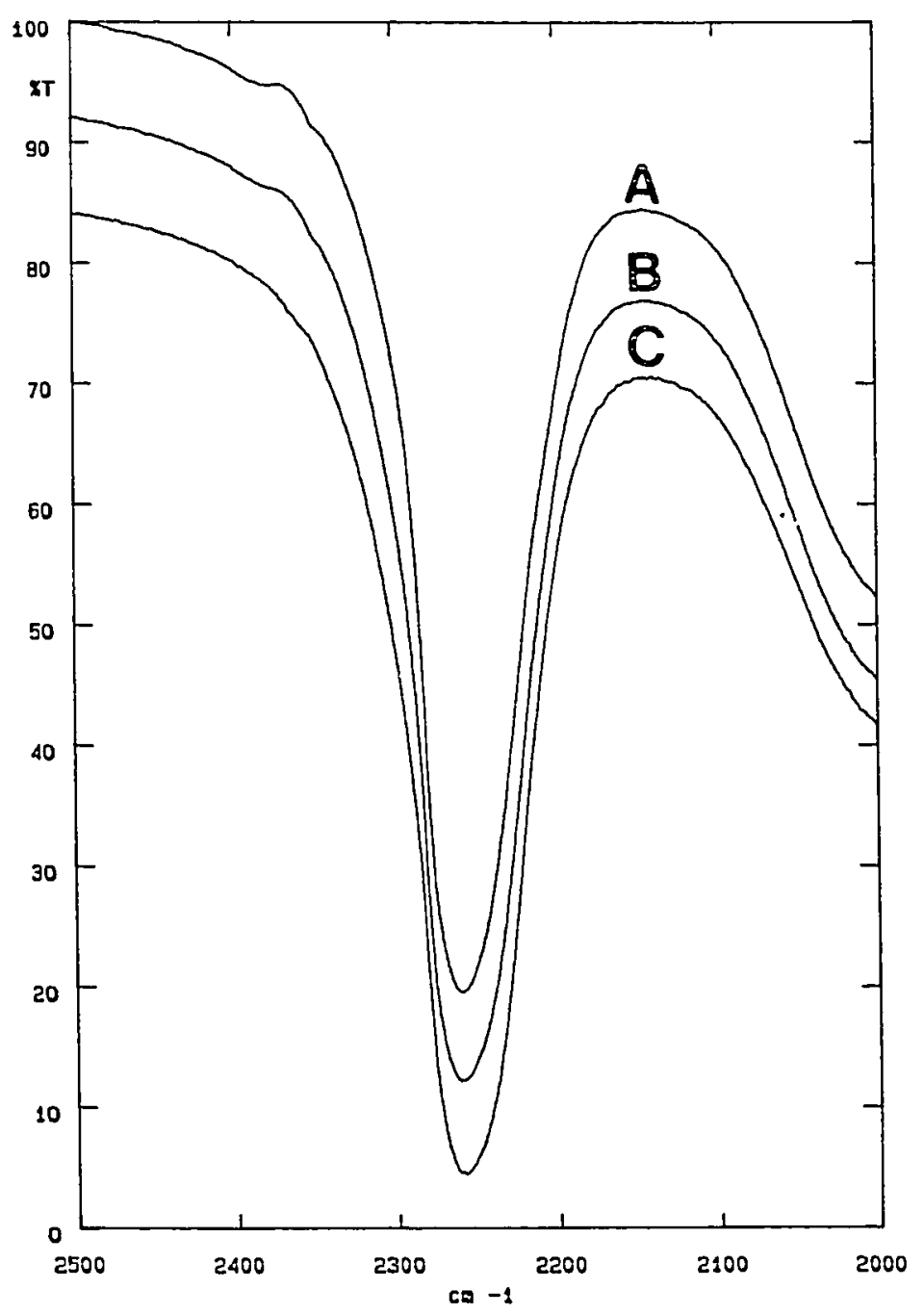

Figure 24. Partial DRIFT spectra (Si-H streching Band) of Hydrosilanized Silica under Varying HCl Concentration: Silica reacted with $0.127 \mathrm{M}$ TES and (A) $0.0010 \mathrm{M}$, (B) $0.010 \mathrm{M}$, (C) $0.10 \mathrm{M} \mathrm{HCl}$ in dioxane under reflux for $60 \mathrm{~min}$. Peaks at (A) $2259 \mathrm{~cm}^{-1}$, (B) $2261 \mathrm{~cm}^{-1}$, (C) $2258 \mathrm{~cm}^{-1}$. 


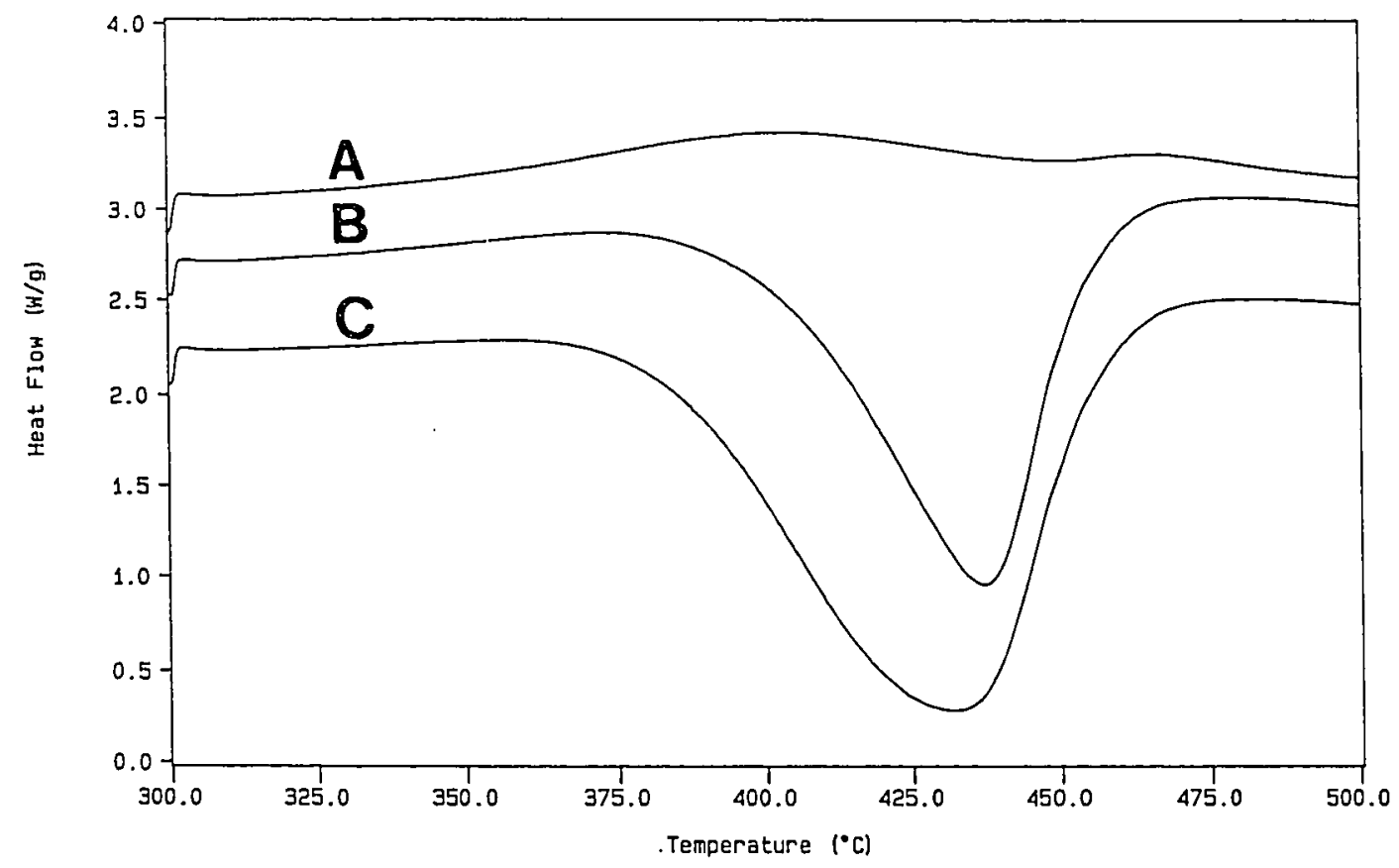

Figure 25. DSC Thermograms of Hydrosilanized Silica at Various $\mathrm{HCl}$ Concentrations: Silica reacted with $0.127 \mathrm{M}$ TES and (A) $1.0 \mathrm{mM}$, (B) $10 \mathrm{mM}$, (C) $100 \mathrm{mM} \mathrm{HCl}$ in dioxane under reflux for $60 \mathrm{~min}$. 
(Figure 24) and $\mathrm{H}_{2}$-displacement/GC data (Figure 23) indicate that hydrosilanization actually took place at the $1.0 \mathrm{mM} \mathrm{HCl}$ concentration level. In order to further examine this finding, the upper temperature in the DSC/air experiment was extended to $730^{\circ} \mathrm{C}$ (the maximum temperature attainable with the Perkin-Elmer DSC-7 instrument). Figure 26 shows the thermooxidation curve obtained. As it could be expected, oxidation of the chemisorbed hydrosilane was only partial at $500{ }^{\circ} \mathrm{C}$. Extension of the upper DSC temperature shows that a rather smooth oxidation was taking place. The oxidation curve showed a maximum at about $465^{\circ} \mathrm{C}$ and ended at about $700^{\circ} \mathrm{C}$. Accurate evaluation of the peak area ( $\Delta \mathrm{H}$ value) should, of cause, encompass a wider temperature range. The unusual behavior of this sample can be related to its relatively low $\mathrm{SiH}$ coverage which might result in a variety of surface SiH species exhibiting various degrees of crosslinking. They might eventually oxidize in a different manner. In any case, an HCl concentration of $0.1 \mathrm{M}$ or above is dictated by the superior coverages obtained.

3.2.5. Suggested Experimental Conditions for the Hydrosilanization of Silica with TES

A set of experimental conditions can be derived from the 


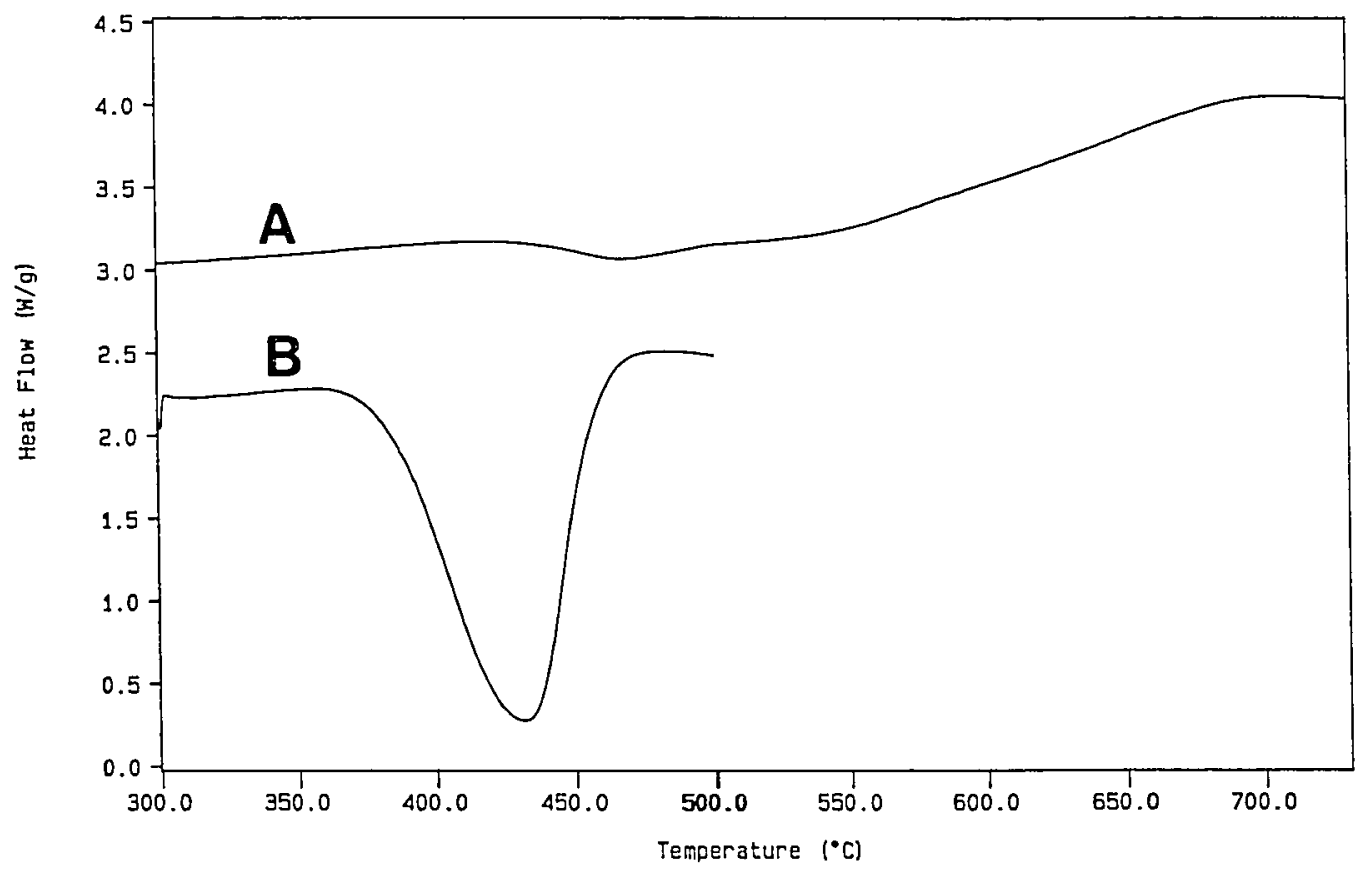

Figure 26. DSC Thermograms of Hydrosilanized Silica at Various $\mathrm{HCl}$ Concentrations: Silica reacted with $0.127 \mathrm{M}$ TES and $1.0 \mathrm{mM} \mathrm{HCl}$ in dioxane under reflux for $60 \mathrm{~min}$. Curves as in Figure 25. Upper DSC temperature for curve (A) was extended to $730^{\circ} \mathrm{C}$. 
experiments described above. These conditions include:

- Use of dioxane as a solvent.

- Reaction temperature corresponding to a gentle reflux (b.p. of dioxane $\left.=101{ }^{\circ} \mathrm{C}\right)$.

- Use of a 10 to $40 \%$ molar excess of TES with respect to available silanols. The total amount of TES used will depend on the nature of the silica support used, particularly surface area and silanol content. Reaction is carried out by slow (dropwise) but continuous addition of TES/dioxane solution into a silica/dioxane suspension containing aqueous catalyst solution.

- A concentration of $\mathrm{HCl}$ catalyst of about $0.1 \mathrm{M}$.

- A reaction time of 40 to 60 minutes.

Under these conditions, a dense hydrosiloxane monolayer should be formed on the silica surface. Further optimization might be required to systematically minimize the population of uncondensed silanols while maintaining a monolayer of hydrosilane species.

\subsubsection{TGA Profiles of Hydrosilanized Silica}

As mentioned in a previous section, when a hydridemodified silica is heated under an inert atmosphere such as dry nitrogen, an unexpected weight increase is observed 
with the TGA above $600^{\circ} \mathrm{C}$. The effect appears to increase with increasing $\mathrm{SiH}$ coverage, as shown in Figure 9 for several samples produced in an early experiment with ethanol solvent (curves $A-C$ in Figure 9 represent materials containing $0.50,0.83$ and $2.70 \mathrm{mmol} \mathrm{SiH} / \mathrm{g}$ respectively). Figure 27 depicts the TGA thermogram of a material prepared using optimum conditions (including dioxane as solvent) and containing $2.98 \mathrm{mmol} \mathrm{SiH} / \mathrm{g}$. At the peak $\left(530-550^{\circ} \mathrm{C}\right)$, the sample shows a relative "weight increase" of about $1 \%$ with respect to the starting value. Notice also that the peak occurs at lower temperature compare to those of Figure 9 (curve C reached its maximum "weight" well after the upper temperature $900^{\circ} \mathrm{C}$ was attained). That the effect was not due to the formation of a silicon nitride was demonstrated by not seeing any change upon switching the nitrogen gas for argon or helium.

To further document this anomalous observation, the same material of Figure 27 was heated under argon and removed from the TGA pan just when the maximum "weight" was reached, that is, about $550{ }^{\circ} \mathrm{C}$. The solid was rapidly mixed with $\mathrm{KBr}$ and its DRIFT spectrum taken. A similar procedure was carried out with another fresh portion of the sample after being subjected to a full TGA heading cycle (up to 900 ${ }^{\circ} \mathrm{C}$ until no weight change was observed). The DRIFT curves are shown in Figure 28 along with that of the starting 

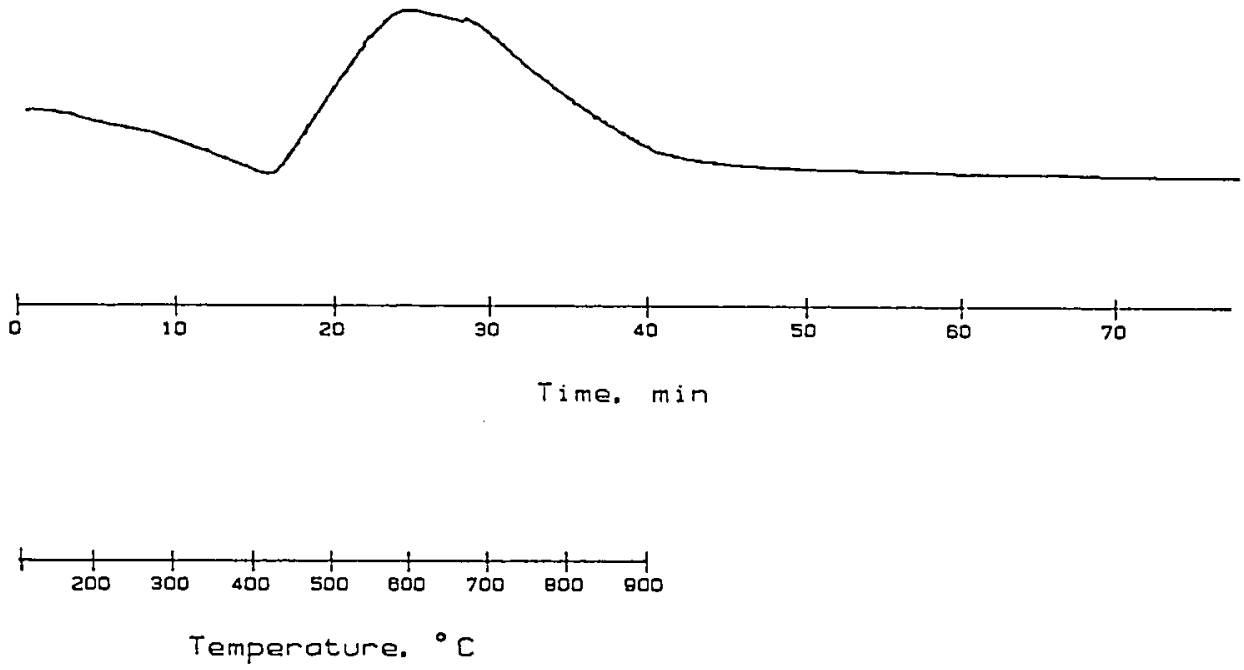

Figure 27. TGA/Argon Curves for Hydrosilanized Partisil-40 Silica reacted with $0.127 \mathrm{M}$ TES, $0.10 \mathrm{M} \mathrm{HCl}$ in dioxane under reflux for $60 \mathrm{~min}$ 


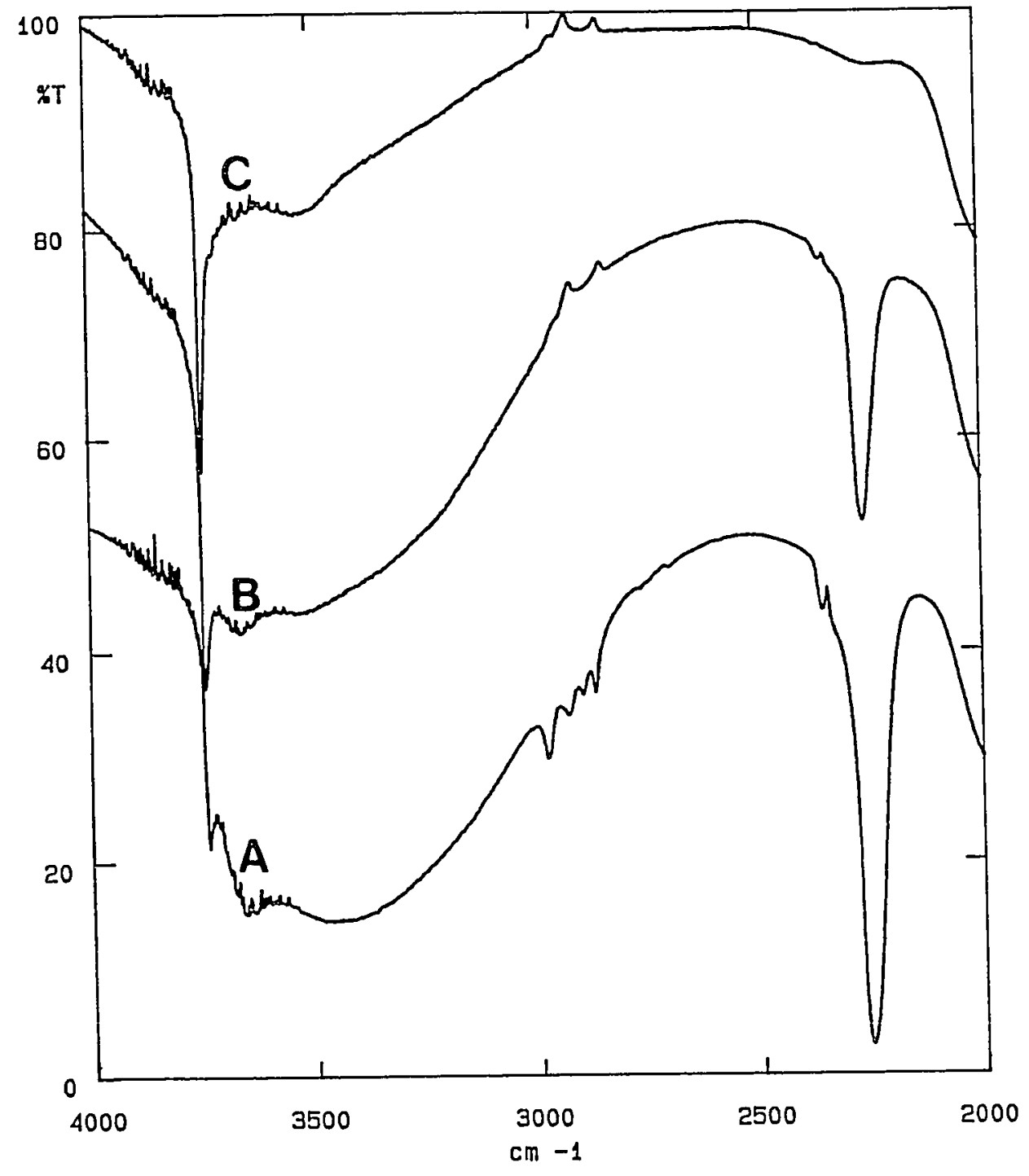

Figure 28. Partial DRIFT Spectra of Hydrosilanized Silica: (A) Starting material; (B) After heating to 530-550 ${ }^{\circ} \mathrm{C}$ under argon; (C) After heating to $900{ }^{\circ} \mathrm{C}$ under argon 
material. It is clear that no species other than the expected were present on the $550{ }^{\circ} \mathrm{C}$ - headed substrate (curve B). It is also evident that at this temperature a significant fraction of the initial SiH population has been decomposed (curve $B$ versus $A$ ). At $900^{\circ} \mathrm{C}$ (curve $\mathrm{C}$ ) no siH appears to be present. Presumably, the two processes occurring at these temperatures, $i . e .$, silanol condensation (equation 16) and hydrosilane decomposition, are accompanied by weight loss only. Since the effect is observable only on materials with high siH coverage (never with any native silica), it seems likely that the observed weight gain is only apparent and that an instrumental artifact occurs with these materials.

A thorough evaluation of this presumed artifact seems beyond the scope of this thesis. It should, however, be pointed out that when siliceous materials such as fused silica (a non-porous solid) are heated (under similar conditions) just above $500^{\circ} \mathrm{C}$, the annealing process is accompanied by a transient increase of the material's electrical conductivity. Such a peculiar behavior of this non-conducting solid has been attributed to the $t^{\prime}$.. nal generation of small concentrations of positive charge -arriers within the $\mathrm{SiO}_{2}$ framework. (21) It seems likely that the observations described above for hydrosilanized silicas are somehow related to the phenomenon occurring when annealing fused 
silicas. Perhaps, a large siH concentration on a large surface enhances the formation of thermally induced charge carriers within the silica matrix. However, without further experimental evidence, it would be highly speculative to attribute the effect to change in electrical conductivity.

3.3. Application of Hydrosilanization with TES on Several Commercial Silicas

Six silica substrates encompassing a wide range of geometries were used to further evaluate the new synthetic method. For comparison purposes, the same substrates were subjected to a chlorination/reduction sequence (equations 8 and 9), as described in section 2.3 .1 .2 (7). Relevant physical and chemical characteristics on the native silica are summarized in Table 7. It should be noticed that the Vydac supports exhibit an unusually high silanol content, as measured by weight-loss on heating (TGA). The generally accepted surface density of silanols is $7-8 \mu \mathrm{mol} / \mathrm{m}^{2}$ for a fully hydroxylated silica $(1,16)$. Such a high silanol density on the surface of Vydac materials can be attributed to a large population of silanols which are either "buried" or inside extremely narrow channels (micropores). Although they are at least partially condensed under TGA temperatures ( $900{ }^{\circ} \mathrm{C}$ in our case), these silanols are not accessi- 
Table 7. Physicochemical Characteristics of Native Silicas used for Hydride-Modification

\begin{tabular}{lrrrr} 
& $\begin{array}{c}\text { Spec. Surf. } \\
\text { Silica }\end{array}$ & \multicolumn{2}{c}{$\begin{array}{c}\text { Mean Pore } \\
\text { Area }^{\mathrm{a}}, \mathrm{m}^{2} / \mathrm{g}\end{array}$} & $\begin{array}{c}\text { Silanol Content } \\
\text { Diam }\end{array}$ \\
\hline Partisil-40 & 315.3 & 90 & 3.32 & 10.5 \\
Partisil-10 & 375.1 & 91 & 3.84 & 10.2 \\
Kromasil-100A & 329.1 & 104 & 2.16 & 6.6 \\
Vydac (890414) & 88.8 & 334 & 4.20 & 47.3 \\
Vydac (890706) & 94.0 & 384 & 4.84 & 51.5 \\
Nucleosil & 110.8 & 368 & 0.84 & 7.6
\end{tabular}

a B.E.T. nitrogen adsorption method

b From TGA data (110-900 ${ }^{\circ} \mathrm{C}$ heating cycle)

ble to most chemical reagents used for surface derivatization. Additionally, water is very strongly adsorbed in micropores, even after prolonged heating at $110^{\circ} \mathrm{C}$. Such water is known to be retained at temperatures as high as 180 ${ }^{\circ} \mathrm{C}$ (16). The actual reason for a high silanol content in Vydac materials is probably a combination of all of the effects above.

Experimental conditions for hydrosilanization were kept as uniform as possible in order to circumvent the problem associated with variability in the silica supports. In all 
cases, a $40 \%$ molar excess of TES with respect to the total silanol content was used. The total volume of dioxane used was adjusted to maintain a final TES concentration of about $150 \mathrm{mM}$, a fairly representative value from previous experiments. Additionally, the volume and concentration of aqueous HCl solution used was adjusted so that the final concentration of the acid was about $100 \mathrm{mM}$ and, at the same time, the amount of water was 12 -fold with respect to the molar amount of TES (see equation 6). Other conditions and/or procedures were as described in previous sections (2.3.1.1.2 and $3 \cdot 2 \cdot 5)$.

Table 8 and 9 show some important physical and chemical characteristics of the hydride-modified supports prepared via chlorination/reduction and hydrosilanization respectiveIy.

As expected from previous experiments, roughly a 4-to 7fold increase in specific $\mathrm{siH}$ coverage is obtained from hydrosilanization with TES. This is illustrated in Figure 29. Perhaps more informative is the $\mathrm{SiH}$ coverage expressed in molar quantity per unit surface area, as illustrated in Figure 30. The "reduced" products show a rather constant SiH surface density. Except for the Vydac materials, hydrosilanized silicas show a surface density of $\mathrm{SiH}$ approaching that of SiOH in the corresponding unmodified substrates. This indicates a high modification efficiency with respect 


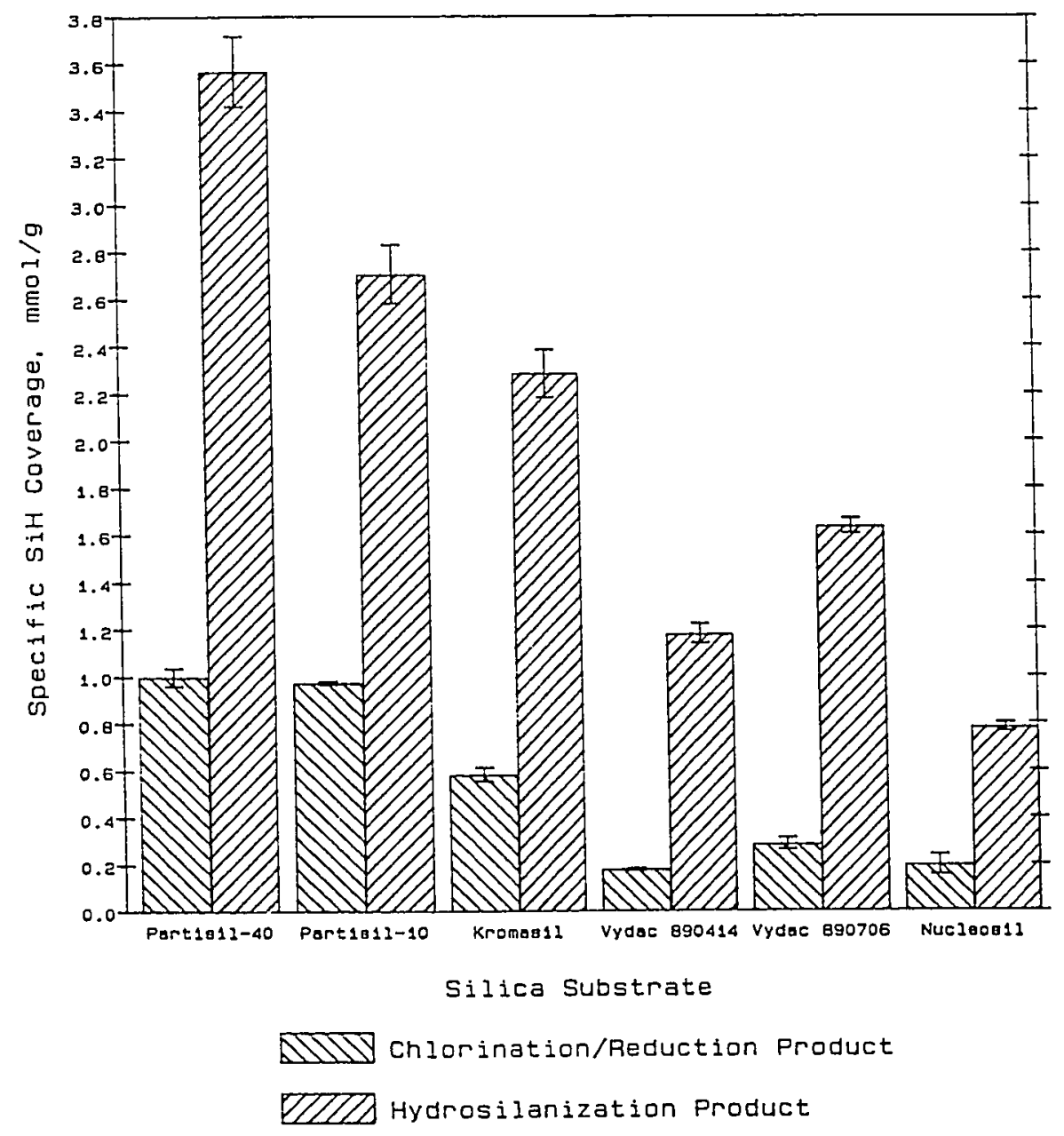

Figure 29. Specific Hydrosilane Coverages for Several Commercial Silicas Subjected to Two Hydride-Modification Methods. 


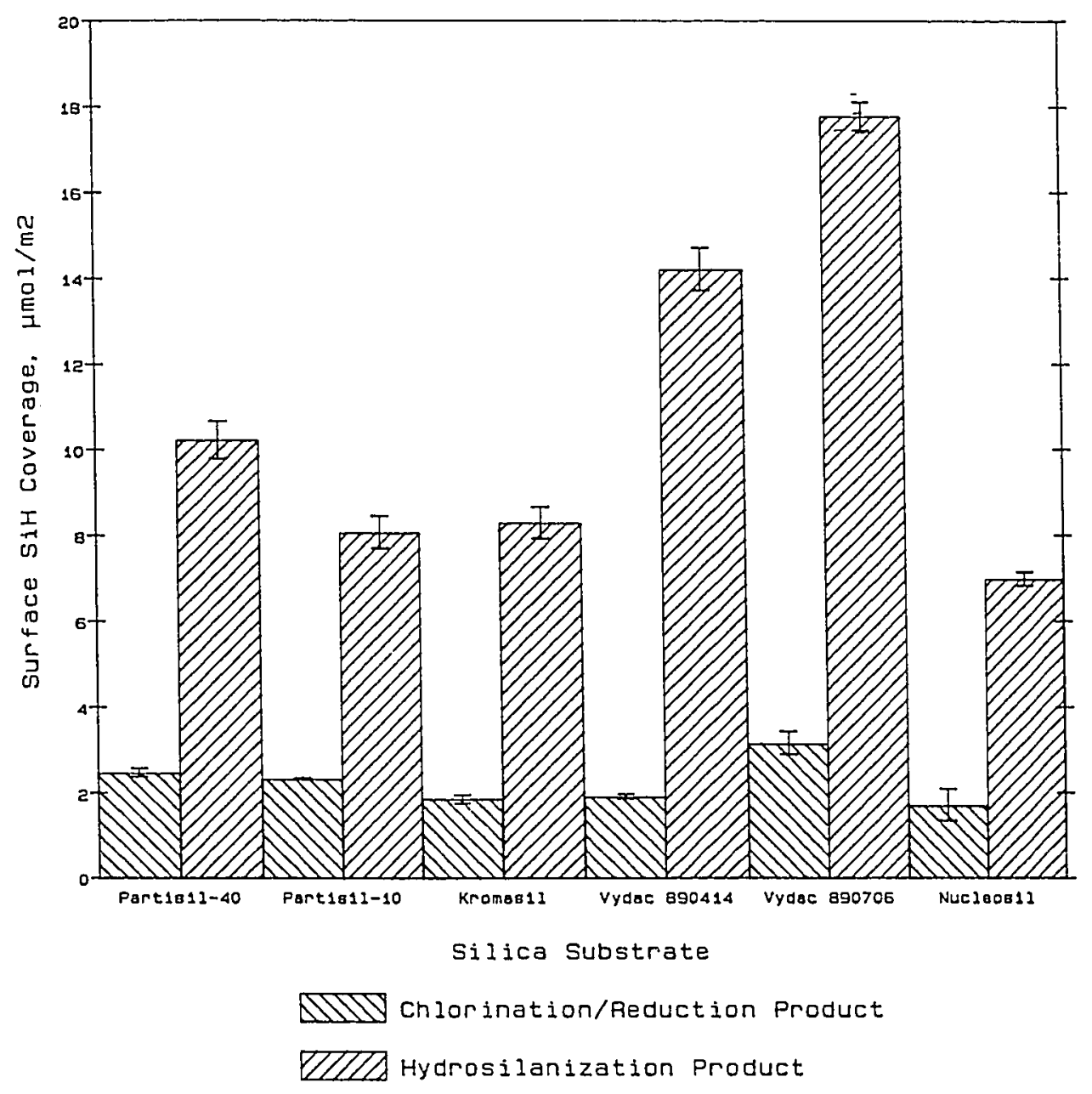

Figure 30. Hydrosilane Surface Density for Several Commercial Silicas Subjected to Two Hydride-Modification Methods. 
Table 8. Physicochemical Characteristics of Hydride-Modified Silica Prepared via a Chlorination/Reduction sequence

Spec. Surf. Mean Pore SiH Content Modif. Silica Area, $\mathrm{m}^{2} / \mathrm{g}$ Diam., $\AA \mathrm{mmol} / \mathrm{g} \mu \mathrm{mol} / \mathrm{m}^{2}$ Eff.,\%

$\begin{array}{lrrrrr}\text { Partisil-40 } & 402.7 & 74 & 0.997 & 2.48 & 24 \\ \text { Partisil-10 } & 416.9 & 94 & 0.972 & 2.33 & 23 \\ \text { Kromasil-100 } & 313.5 & 109 & 0.583 & 1.86 & 28 \\ \text { Vydac (890414) } & 93.4 & 317 & 0.178 & 1.91 & 4 \\ \text { Vydac (890706) } & 90.5 & 391 & 0.286 & 3.16 & 6 \\ \text { Nucleosil 300-10 } & 113.5 & 346 & 0.194 & 1.71 & 23\end{array}$

Table 9. Physicochemical Characteristics of Hydride-Modified Silicas Prepared via Hydrosilanization with TES

Spec. Surf. Mean Pore SiH Content Modif. \%C Silica Area, $\mathrm{m}^{2} / \mathrm{g}$ Diam, $\AA$ mmol $/ \mathrm{g} \mu \mathrm{mol} / \mathrm{m}^{2}$ Eff., $\%$

$\begin{array}{lrrrrrr}\text { Partisil-40 } & 348.6 & 76 & 3.57 & 10.2 & 97 & 0.51 \\ \text { Partisil-10 } & 334.8 & 90 & 2.71 & 8.1 & 79 & 0.43 \\ \text { Kromasil-100A } & 275.2 & 106 & 2.29 & 8.3 & 126 & 0.49 \\ \text { Vydac (890414) } & 82.9 & 278 & 1.18 & 14.2 & 30 & 0.91 \\ \text { Vydac (890706) } & 91.9 & 344 & 1.64 & 17.8 & 35 & 0.84 \\ \text { Nucleosil 300-10 } & 111.7 & 360 & 0.78 & 7.0 & 92 & 0.16\end{array}$


to the starting silanol content, as expected from previous experiments on Partisil-40. Although Vydac materials show a lower efficiency value, the number of SiH groups per unit surface area exceeds the expected (normal) 7-8 $\mu \mathrm{mol} / \mathrm{m}^{2}$ value. Since a large fraction of the total silanols is not accessible to interaction with the hydrosilanetriol species, $\mathrm{HSi}(\mathrm{OH})_{3}$, this finding suggests the formation of some polymeric $\left(\mathrm{HSIO}_{3 / 2}\right)_{\mathrm{n}}$ material. The thermooxidative behavior of the hydride-modified silicas is shown in Figures 31-36. The more extensive hydrosilane coverage of the hydrosilanized product is confirmed from a more intense oxidation peak (curves B). With the exception of the two Vydac silicas, the oxidation peaks of the chlorination/reduction products (curves B) are very broad although easily observed. Both Vydac silicas show multiple, relatively sharp oxidation peaks for the chlorination/reduction product, indicating a more complex hydrosilane population. In contrast with the other silicas, which show a single peak near $450^{\circ} \mathrm{C}$, hydrosilanized Vydacs show a second peak at a lower temperature. This observation appears to verify the formation of some polymeric material which is known to oxidate at lower temperatures (see Figure $5(C)$ and 11).

$\bar{A}$ comparison of the DRIFT spectra of the hydride-modified substrates is illustrated in Figures 37-42. The most obvious feature of the spectra is a stronger si-H stretching 


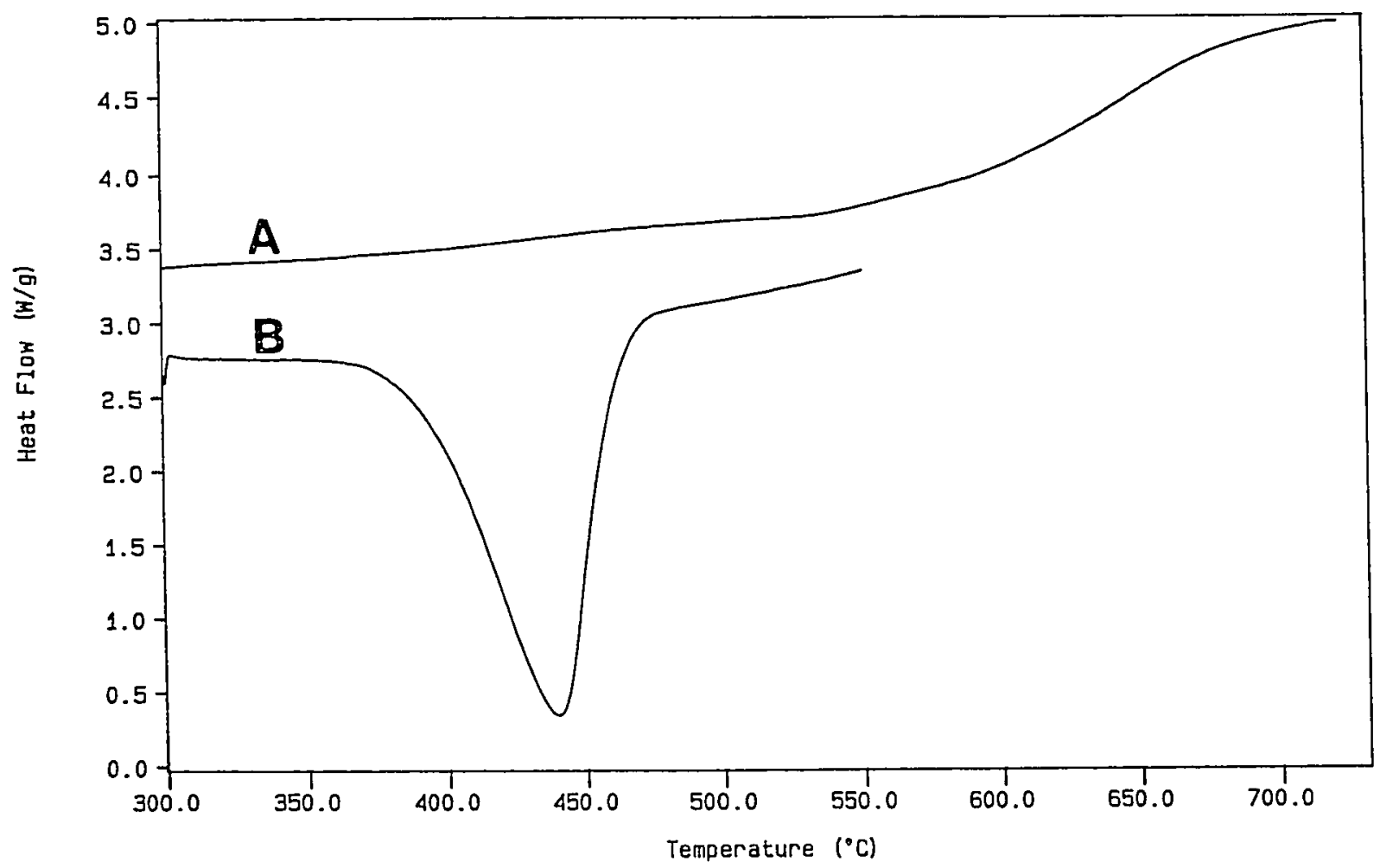

Figure 31. DSC/Air Oxidation Curves of Hydride-Modified Partisil-40 Silica: (A) Chlorination/reduction product; (B) Hydrosilanization product (peak at $440^{\circ} \mathrm{C}$ ) 


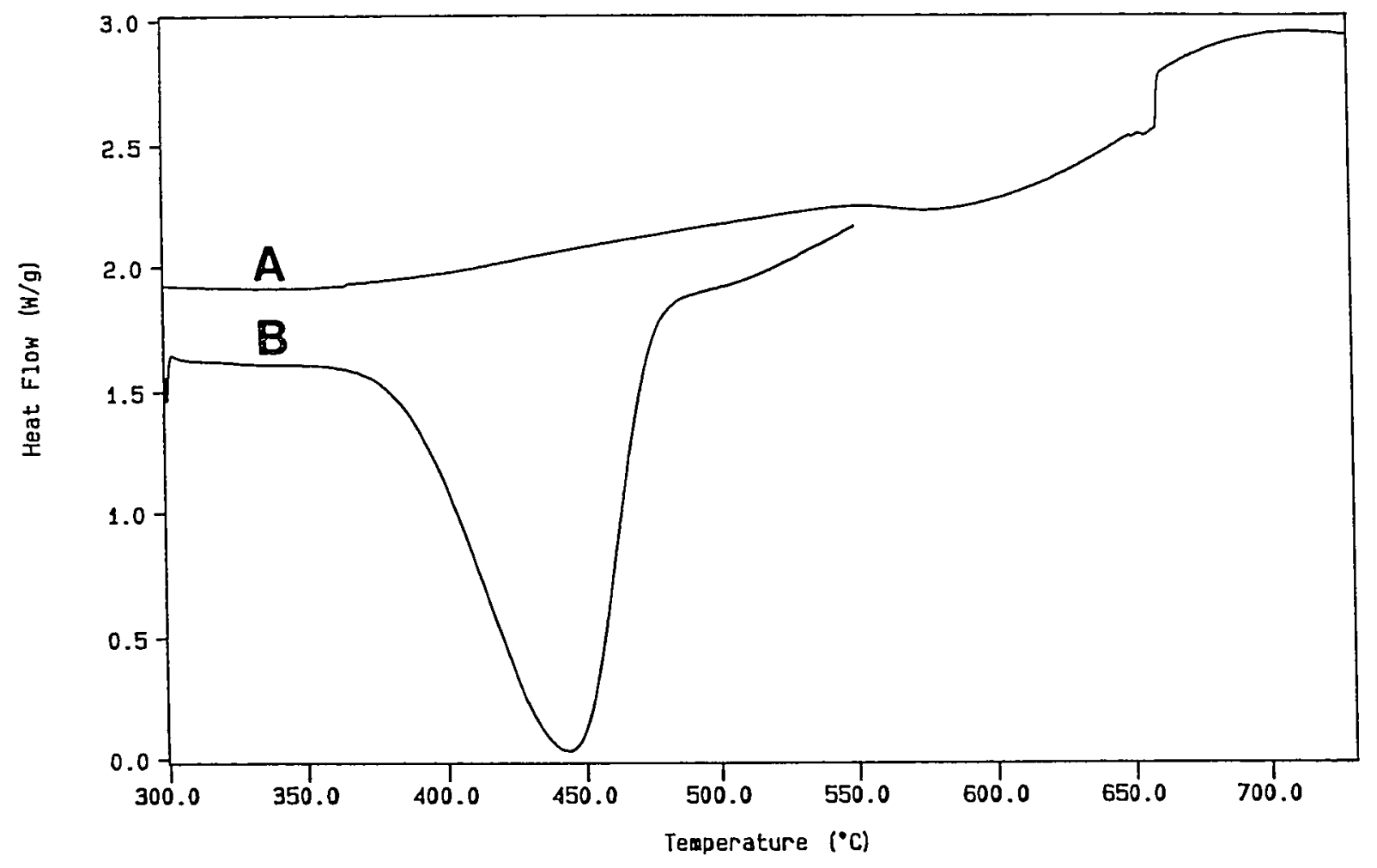

Figure 32. DSC/Air Oxidation Curves of Hydride-Modified Partisil-10 Silica: (A) Chlorination/reduction product; (B) Hydrosilanization product (peak at $444^{\circ} \mathrm{C}$ ) 


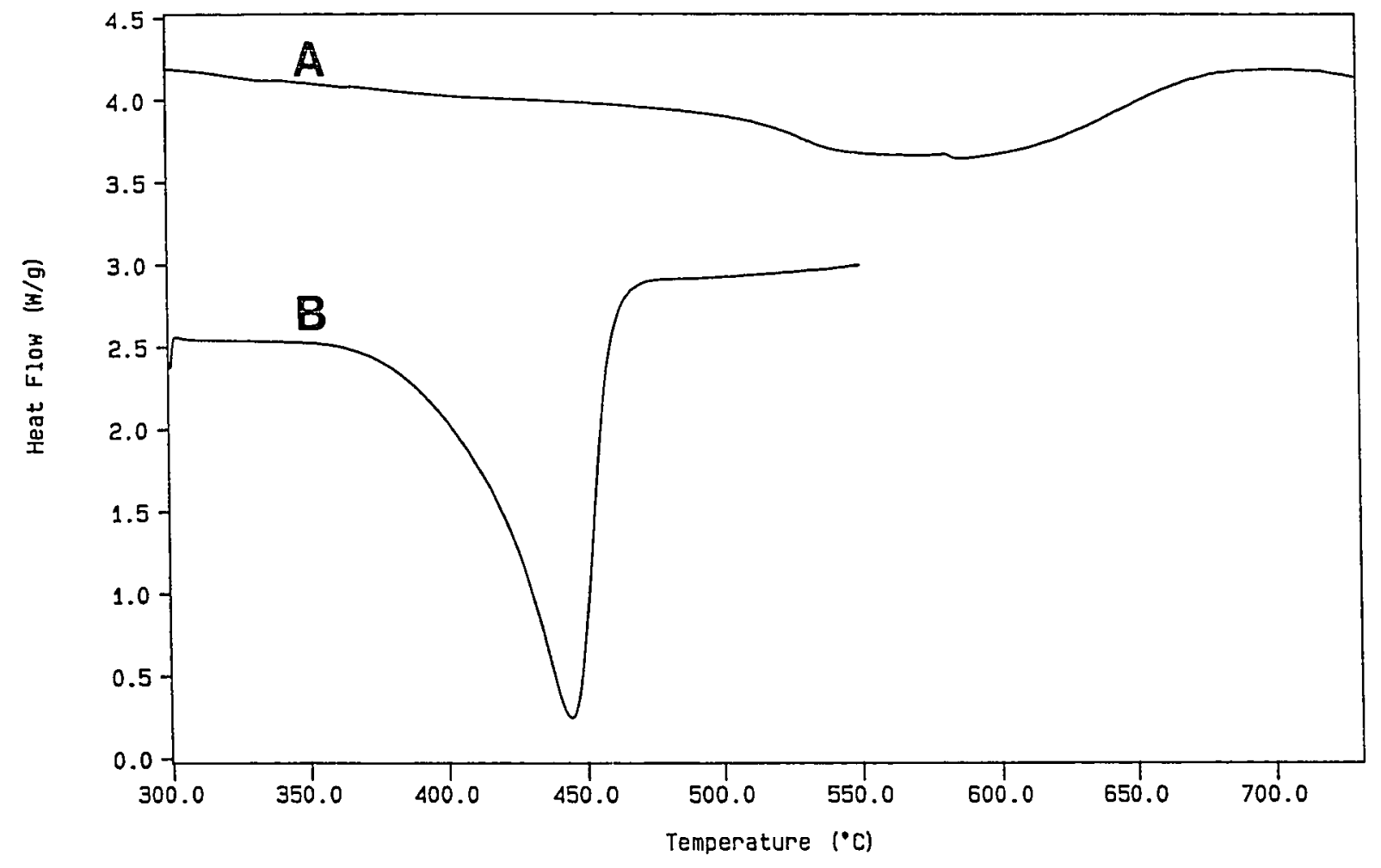

Figure 33. DSC/Air Oxidation Curves of Hydride-Modified Rromasil silica: (A) Chlorination/reduction product; (B) Hydrosilanization product (peak at $445^{\circ} \mathrm{C}$ ) 


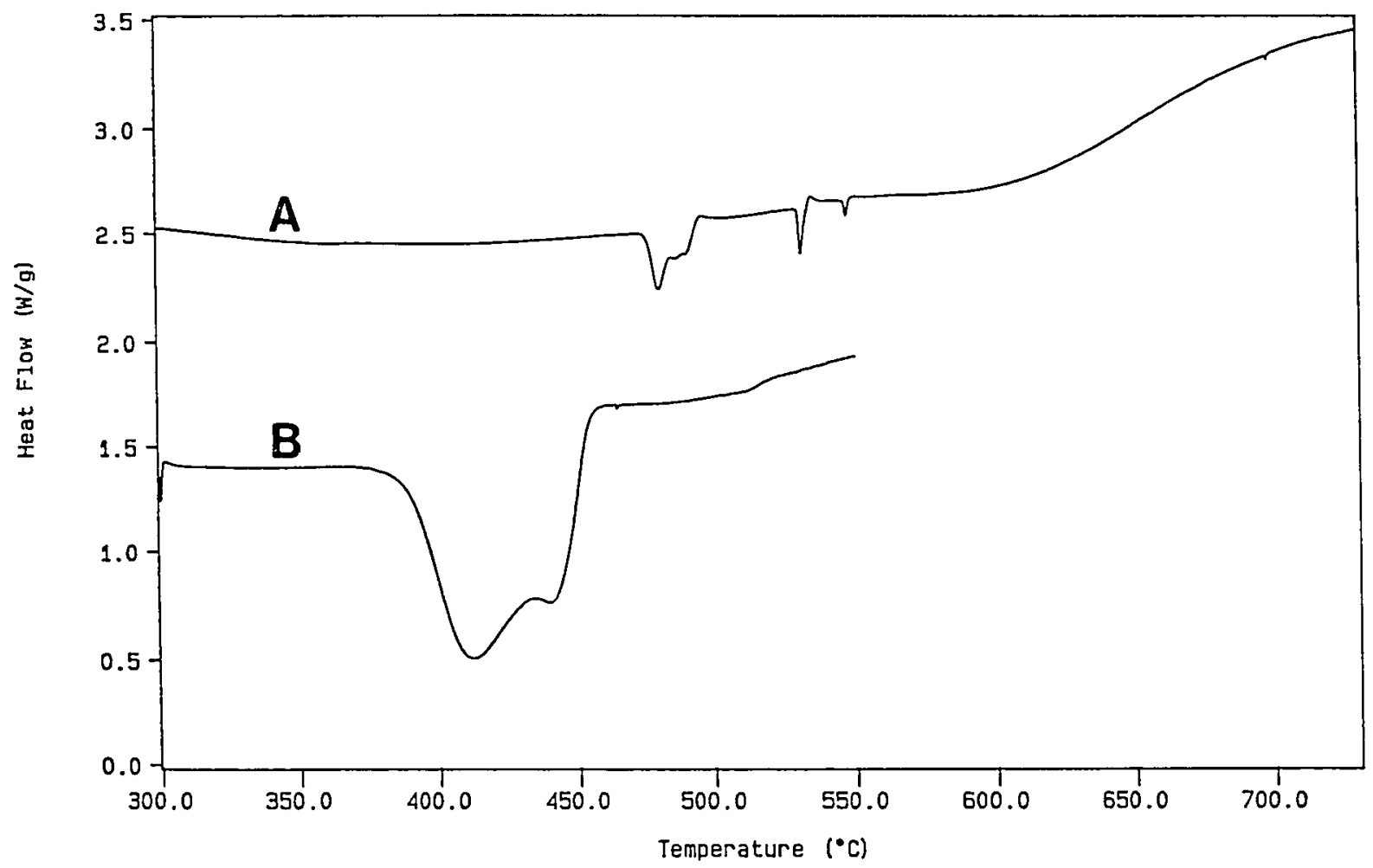

Figure 34. DSC/Air Oxidation Curves of Hydride-Modified Vydac-lot 890414 Silica: (A) Chlorination/reduction product (peaks at 481 (+ shoulder), 531 and $\left.547^{\circ} \mathrm{C}\right) ;(\mathrm{B})$ Hydrosilanization product (peaks at 412 and $440^{\circ} \mathrm{C}$ ) 


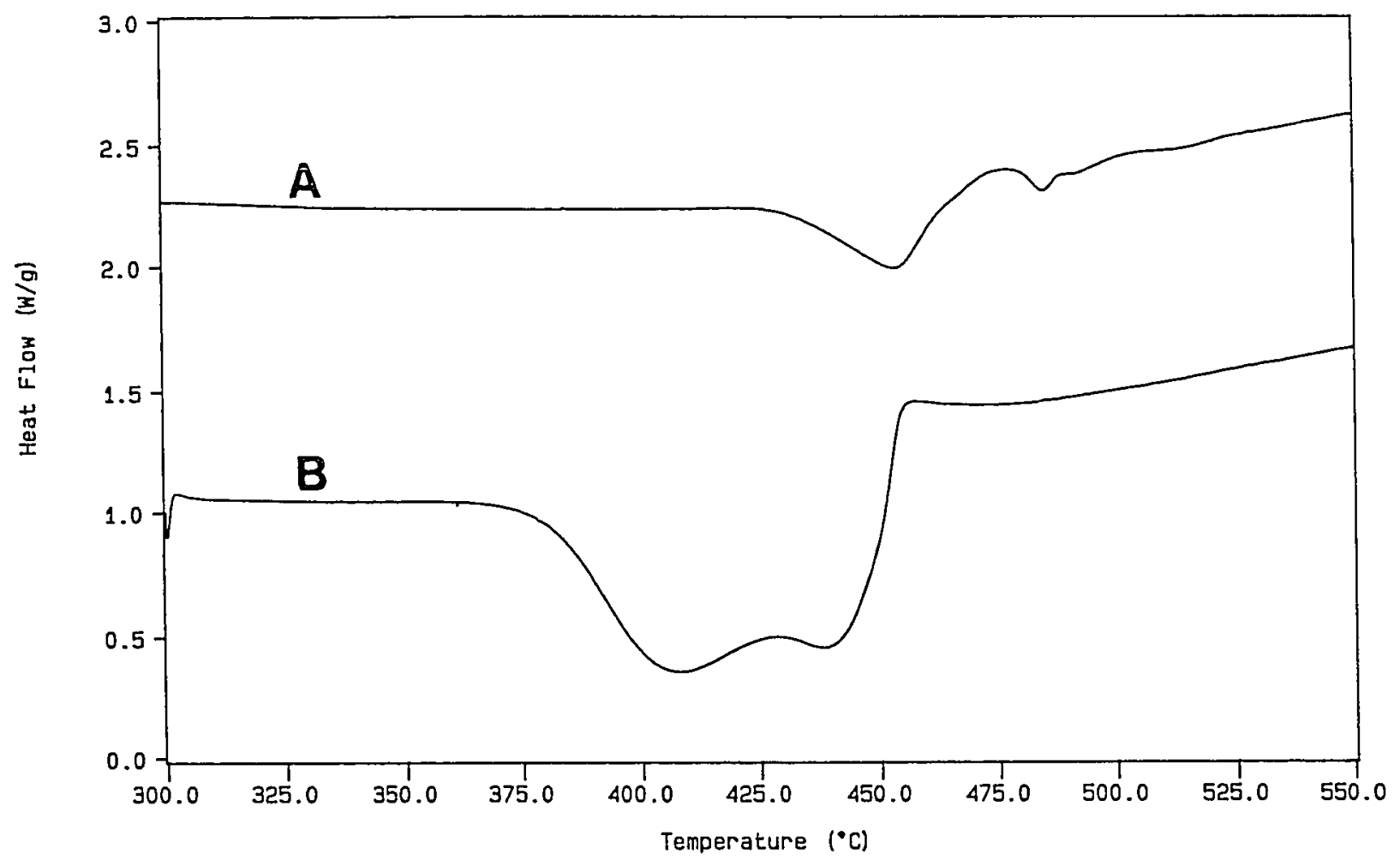

Figure 35. DSC/Air Oxidation Curves of Hydride-Modified Vydac-lot 890706 Silica: (A) Chlorination/reduction product (peaks at 453 and $485^{\circ} \mathrm{C}$ ); (B) Hydrosilanization product (peaks at 407 and $438{ }^{\circ} \mathrm{C}$ ) 


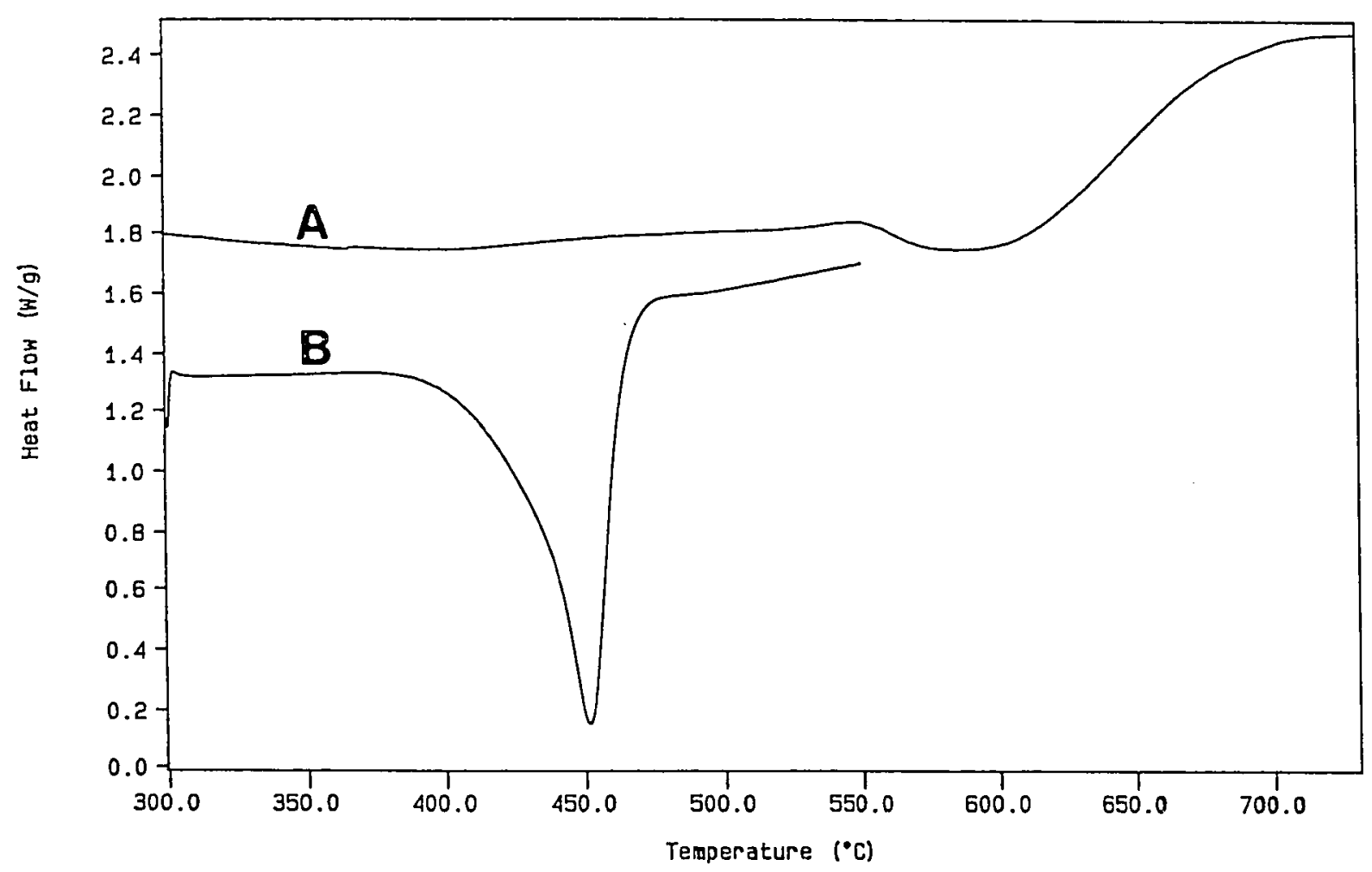

Figure 36. DSC/Air Oxidation Curves of Hydride-Modified Nucleosil Silica: (A) Chlorination/reduction product(peak at $596^{\circ} \mathrm{C}$ ); (B) Hydrosilanization product (peak at $451{ }^{\circ} \mathrm{C}$ ) 


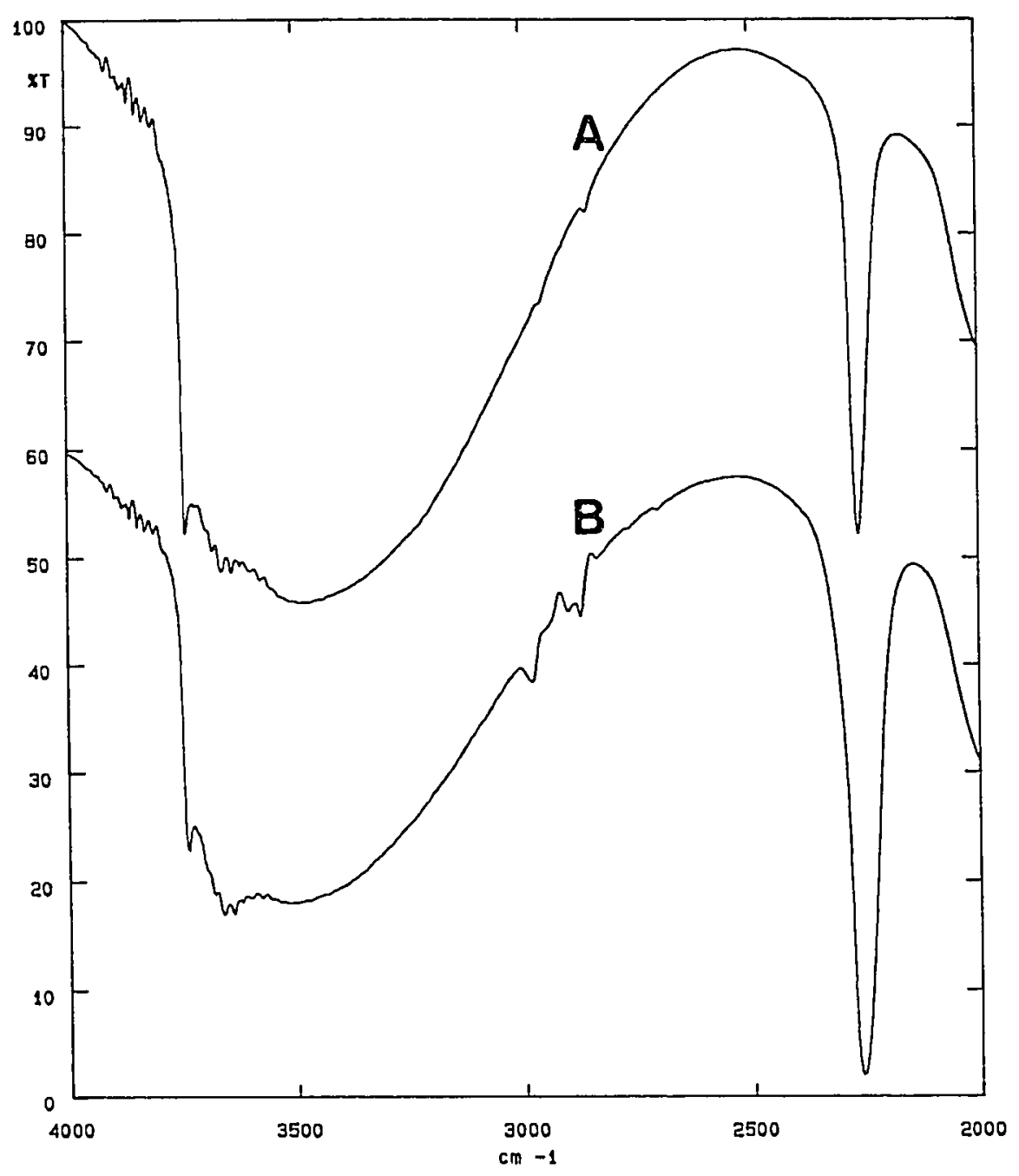

Figure 37. Partial DRIFT Spectra of Hydride-Modified Partisil-40 Silicas: (A) Chlorination/reduction product; (B) Hydrosilanization product. SiO-H and Si-H stretching peaks at (A) 3740 and $2261 \mathrm{~cm}^{-1} ;$ (B) 3736 and $2259 \mathrm{~cm}^{-1}$. 


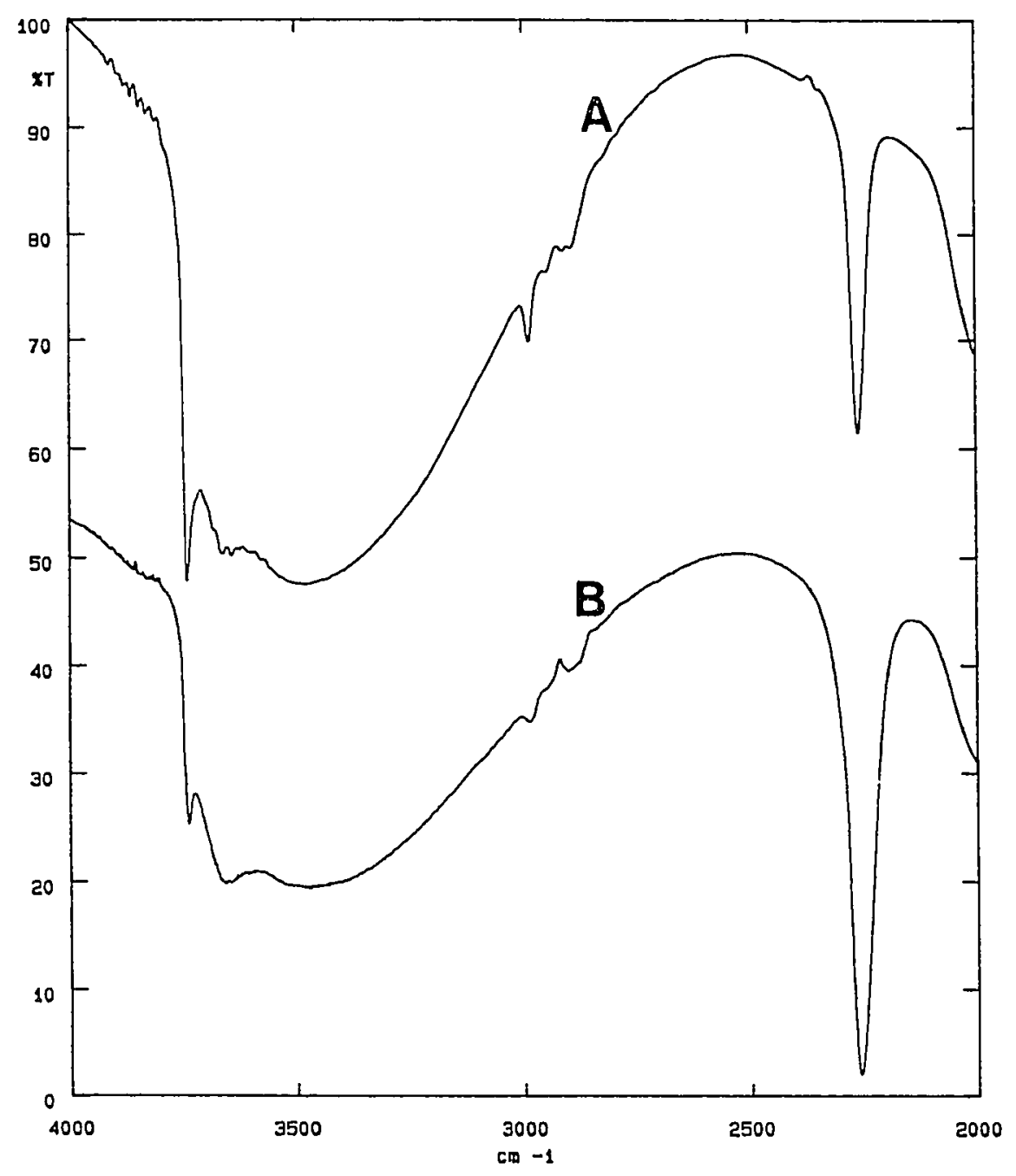

Figure 38. Partial DRIFT Spectra of Hydride-Modified Partio sil-10 silicas: (A) Chlorination/reduction product; (B) Hydrosilanization product. SiO-H and $\mathrm{Si}-\mathrm{H}$ stretching peaks at (A) 3740 and $2259 \mathrm{~cm}^{-1}$; (B) 3739 and $2259 \mathrm{~cm}^{-1}$. 


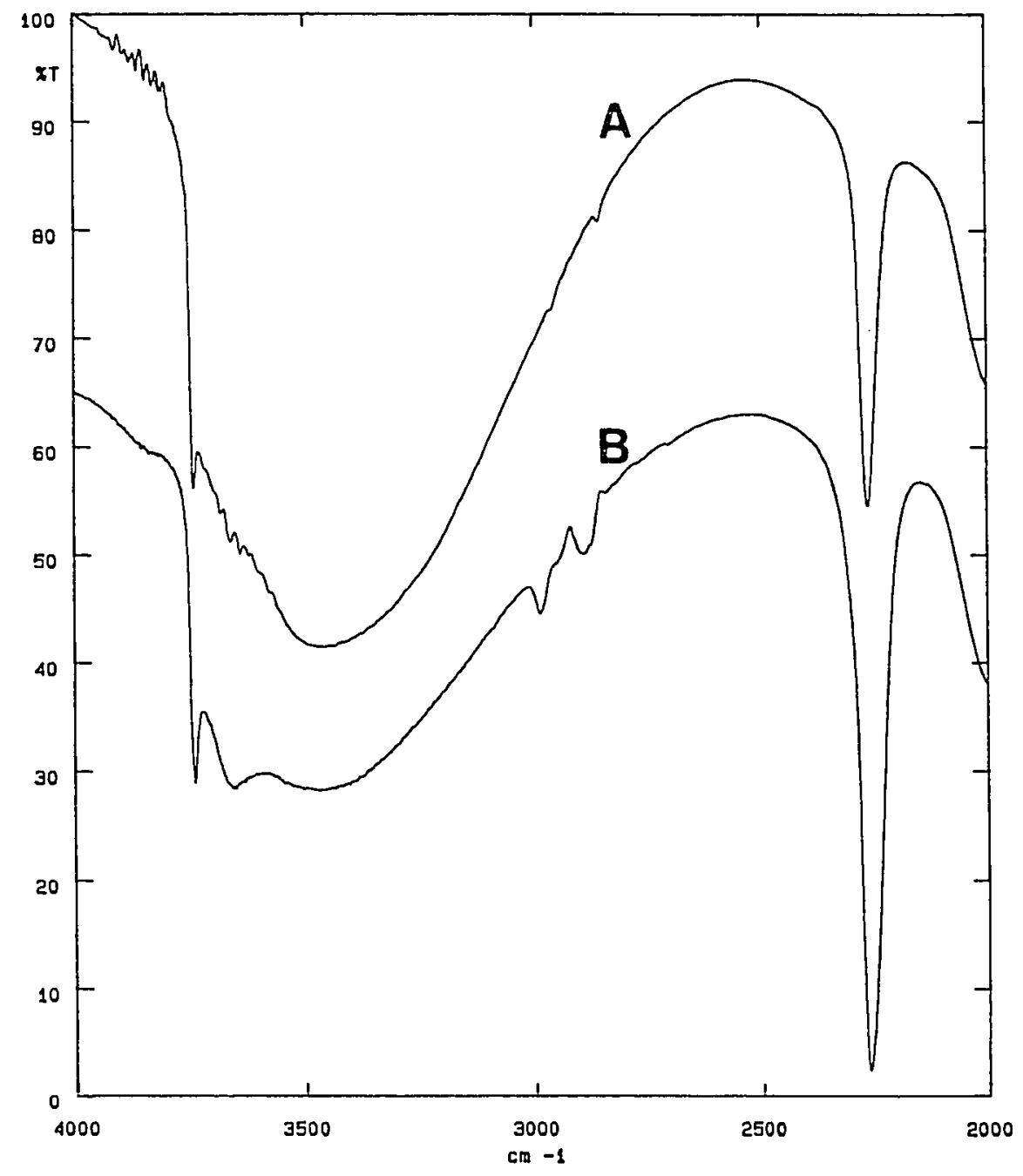

Figure 39. Partial DRIFT Spectra of Hydride-Modified Rromasil Silicas: (A) Chlorination/reduction product; (B) Hydrosilanization product. SiO-H and Si-H stretching peaks at (A) 3741 and $2261 \mathrm{~cm}^{-1} ;$ (B) 3739 and $2259 \mathrm{~cm}^{-1}$. 


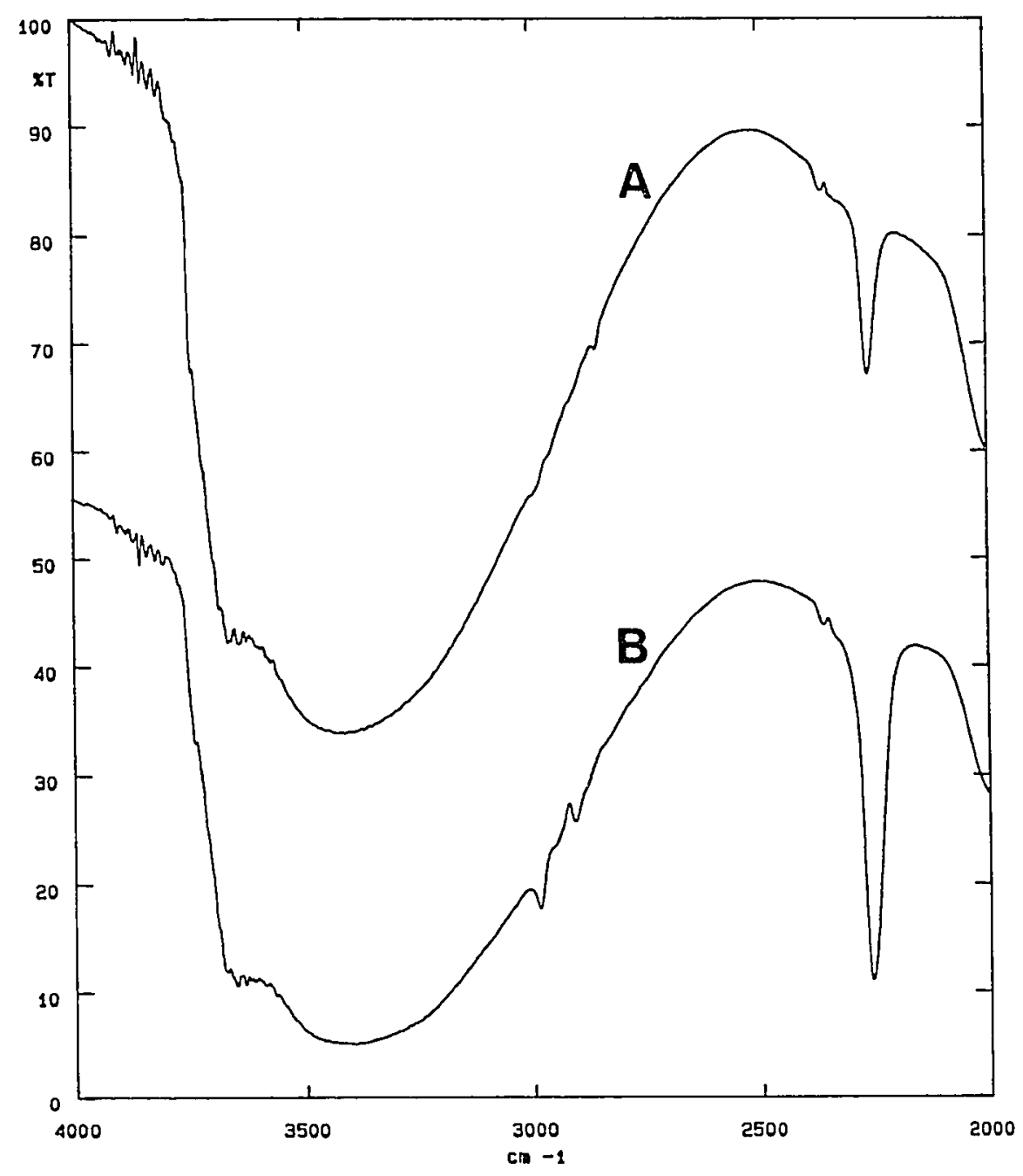

Figure 40. Partial DRIFT Spectra of Hydride-Modified VydacLot $890 \$ 14$ Silicas: (A) Chlorination/reduction product; (B) Hydrosilanization product. SiO-H and $\mathrm{Si}-\mathrm{H}$ stretching peaks at (A) Non visible and $2261 \mathrm{~cm}^{-1} ;$ (B) Non visible and 2258 $\mathrm{cm}^{-1}$. 


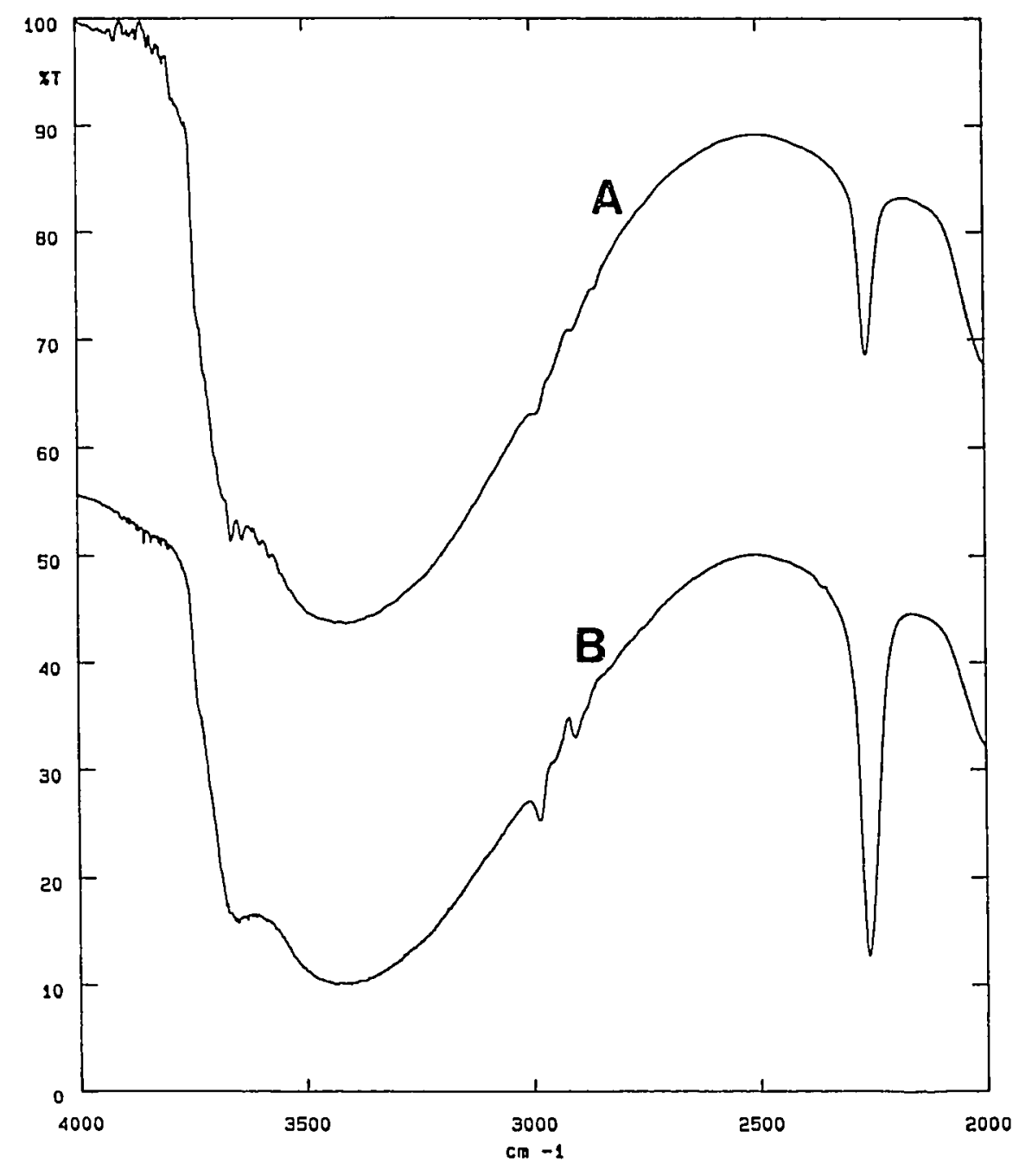

Figure 41. Partial DRIFT Spectra of Hydride-Modified VydacLot 890706 Silicas: (A) Chlorination/reduction product; (B) Hydrosilanization product. SiO-H and $\mathrm{Si}-\mathrm{H}$ stretching peaks at (A) Non visible and $2260 \mathrm{~cm}^{-1} ;$ (B) Non visible and $2259 \mathrm{~cm}^{-1}$. 


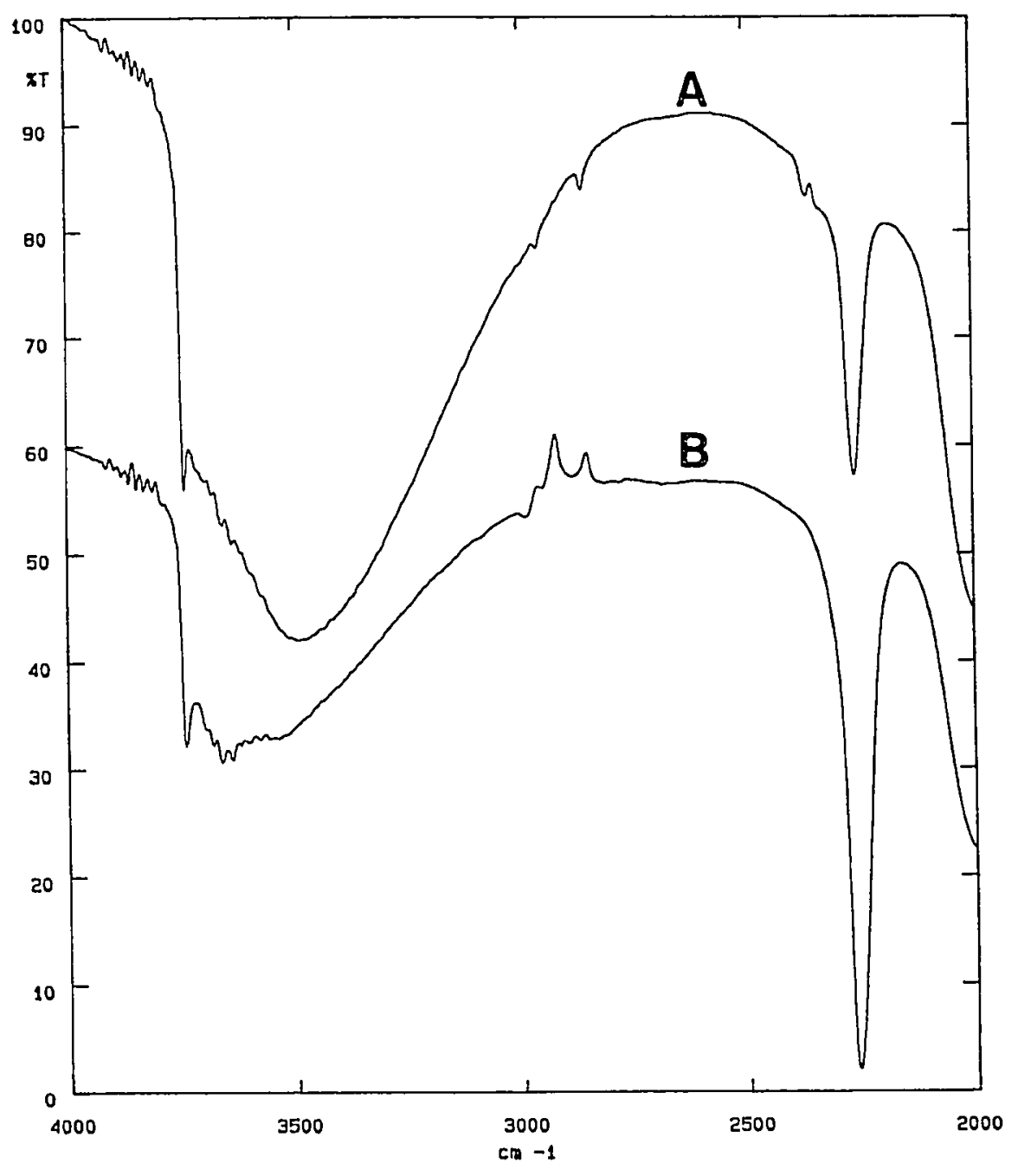

Figure 42. Partial DRIFT Spectra of Hyaride-Modified Nucleosil Silicas: (A) Chlorination/reduction product; (B) Hydrosilanization product. SiO-H and $\mathrm{Si}-\mathrm{H}$ stretching peaks at (A) 3741 and $2260 \mathrm{~cm}^{-1} ;$ (B) 3740 and $2260 \mathrm{~cm}^{-1}$. 
band for the TES deposited material. Also important is the observation that, without expection, the intensity of the isolated silanol stretching band near $3740 \mathrm{~cm}^{-1}$ is smaller for the hydrosilanized materials (curves B). This fact indicates that the new procedure does not contribute additional silanols to the hydrided product. on the contrary, hydrosilanization with TES under the conditions employed appears to occur with extensive silanol removal via condensation (equation 10).

Figures 43 and 44 depict the effect of the hydridemodification methods on surface areas and pore sizes respective1y. It should be recalled that precision of these measurement by the BET method is generally about $5 \%$ but can be as large as $15 \%$. Notice, for instance, that both Partisil materials show an apparent increase in surface area upon chlorination/reduction. Since this modification necessarily occurs at the surface only, no significant increase in surface area should be expected. Presumably, the apparent high value should arise from a relatively large BET uncertainty. It can, therefore, be concluded from Figures 43 and 44 that no significant changes in the pore structure of the substrate appear to occur during hydride-modification. 


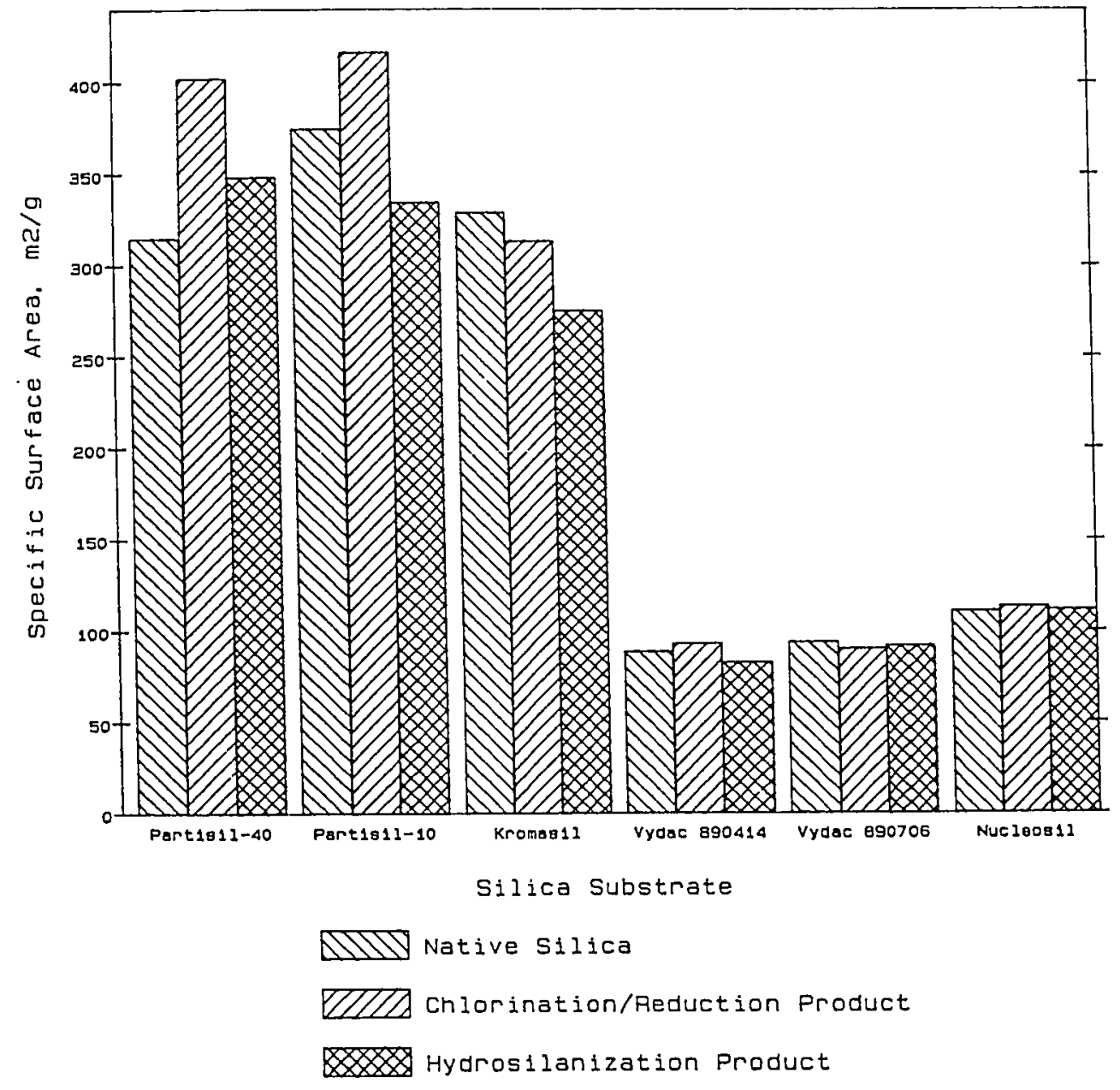

Figure 43. Effect of Hydride-Modification on Specific Surface Area for Several Commercial Silica Supports 


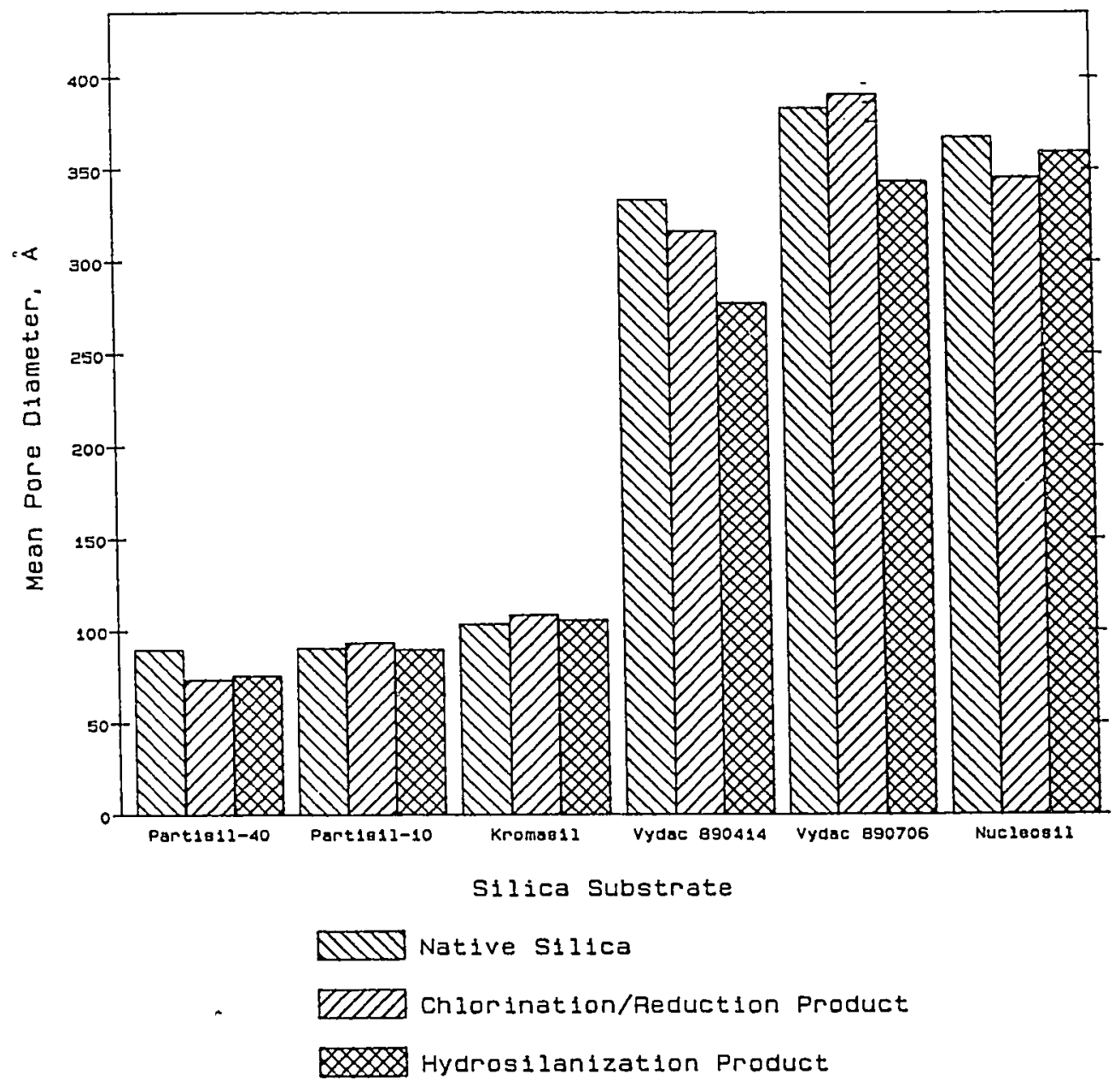

Figure 44. Effect of Hydride-Modification on Pore Size for Several commercial silica supports 


\subsection{Bonded Phases from Hydrosilanized Silicas}

Octyl-bonded silica packings were prepared from the new hydride intermediates by reacting them with 1-octene in the presence of a platinum(II) catalyst as described in section 2.3.1.3. Surface coverage data for the bonded silicas are summerized in Table 10. Calculation of octyl surface concentration was carried out using the following equation developed by Berendsen and De Galan $(17,22)$ :

$$
\alpha_{\mathrm{R}}\left(\mu \mathrm{mol} / \mathrm{m}^{2}\right)=10^{6} \mathrm{P}_{\mathrm{C}} /\left(10^{2} \mathrm{M}_{\mathrm{C}} \mathrm{N}_{\mathrm{C}}-\mathrm{P}_{\mathrm{C}} \mathrm{M}_{\mathrm{R}}\right) \mathrm{S}_{\mathrm{BET}}
$$

where $\mathrm{P}_{C}$ is the carbon percent by weight of the bonded product (corrected from any carbon present before bonding), $\mathrm{N}_{\mathrm{C}}$ is the number of carbon atoms in the olefin, $\mathrm{M}_{\mathrm{R}}$ is the molecular weight of the olefin, $\mathbb{M}_{C}$ is the atomic weight of carbon and $\mathrm{S}_{\mathrm{BET}}$ is the specific surface area of the hydride intermediate. The octyl-coverage values obtained are comparable to those from conventional organosilanization (roughly 3-4 $\left.\mu \mathrm{mol} / \mathrm{m}^{2}\right)(22-24)$. Figures $45-50$ show the DRIFT spectral changes taking place during the derivatization of several commercial silicas, starting from the native substrate (curve A), through the TES-hydrosilanized intermediate (curve B), to the octyl-bonded product (curve C). The appearance of strong strectching bands at $3000-2800 \mathrm{~cm}^{-1}$ 


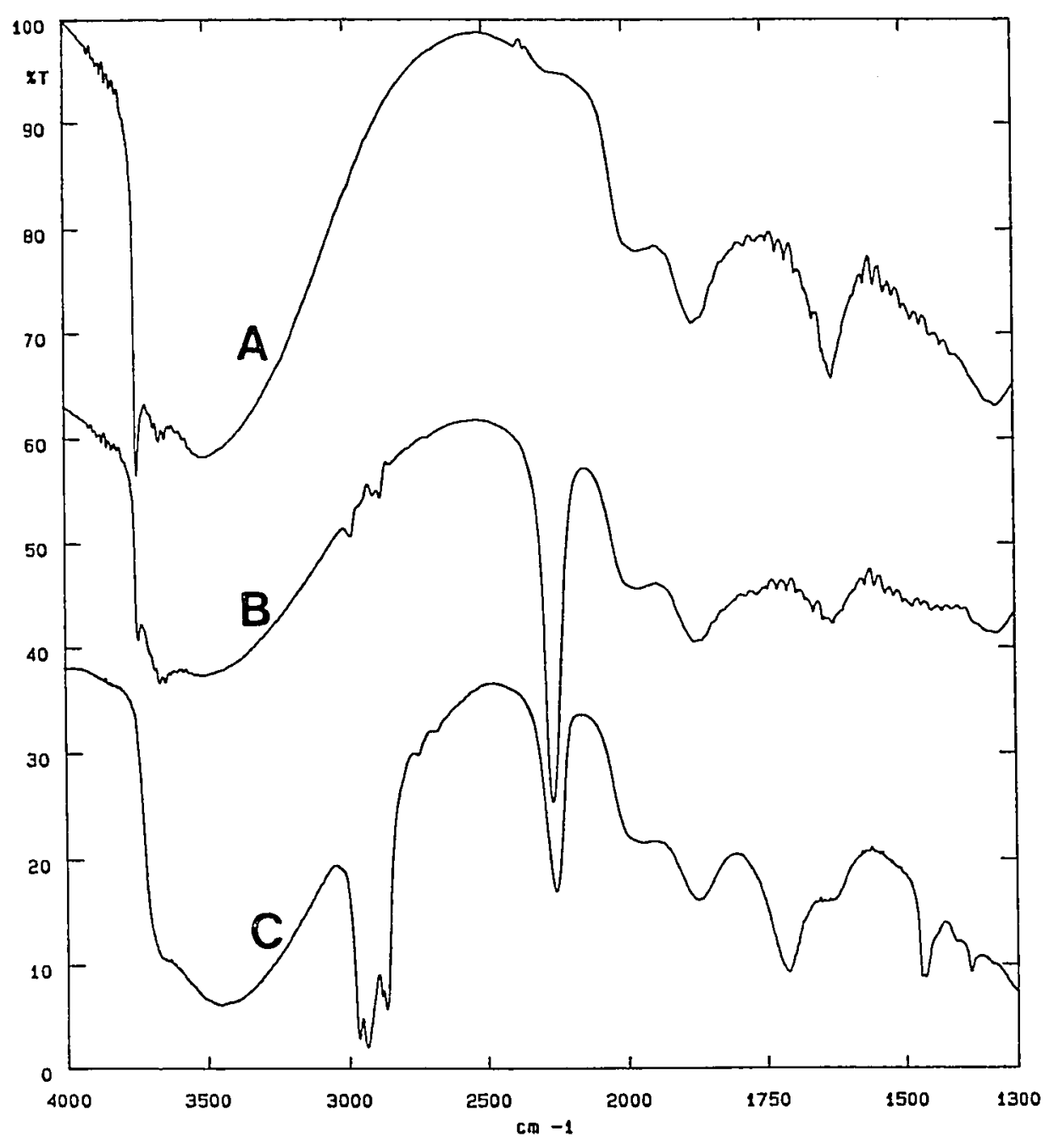

Figure 45. Change in the Partial DRIFT Spectra of Partisil-40 Silica upon Derivatization (A) Native silica; (B) Hydride intermediate prepared via hydrosilanization; (C) Octyl-bonded phase. 


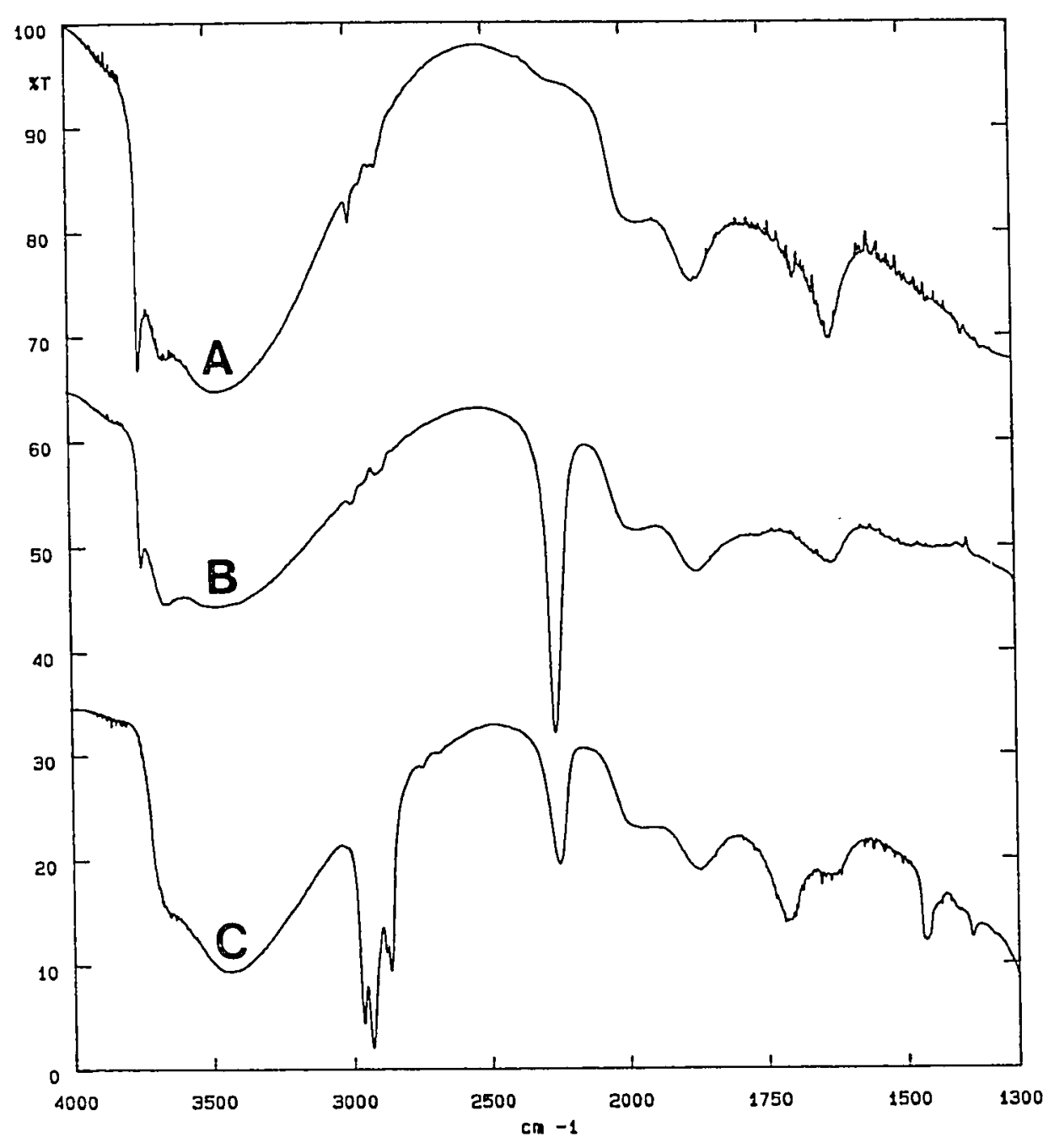

Figure 46. Change in the Partial DRIFT Spectra of Partisil-10 Silica upon Derivatization (A) Native silica; (B) Hydride intermediate prepared via hydrosilanization; (C) Octyl-bonded phase. 


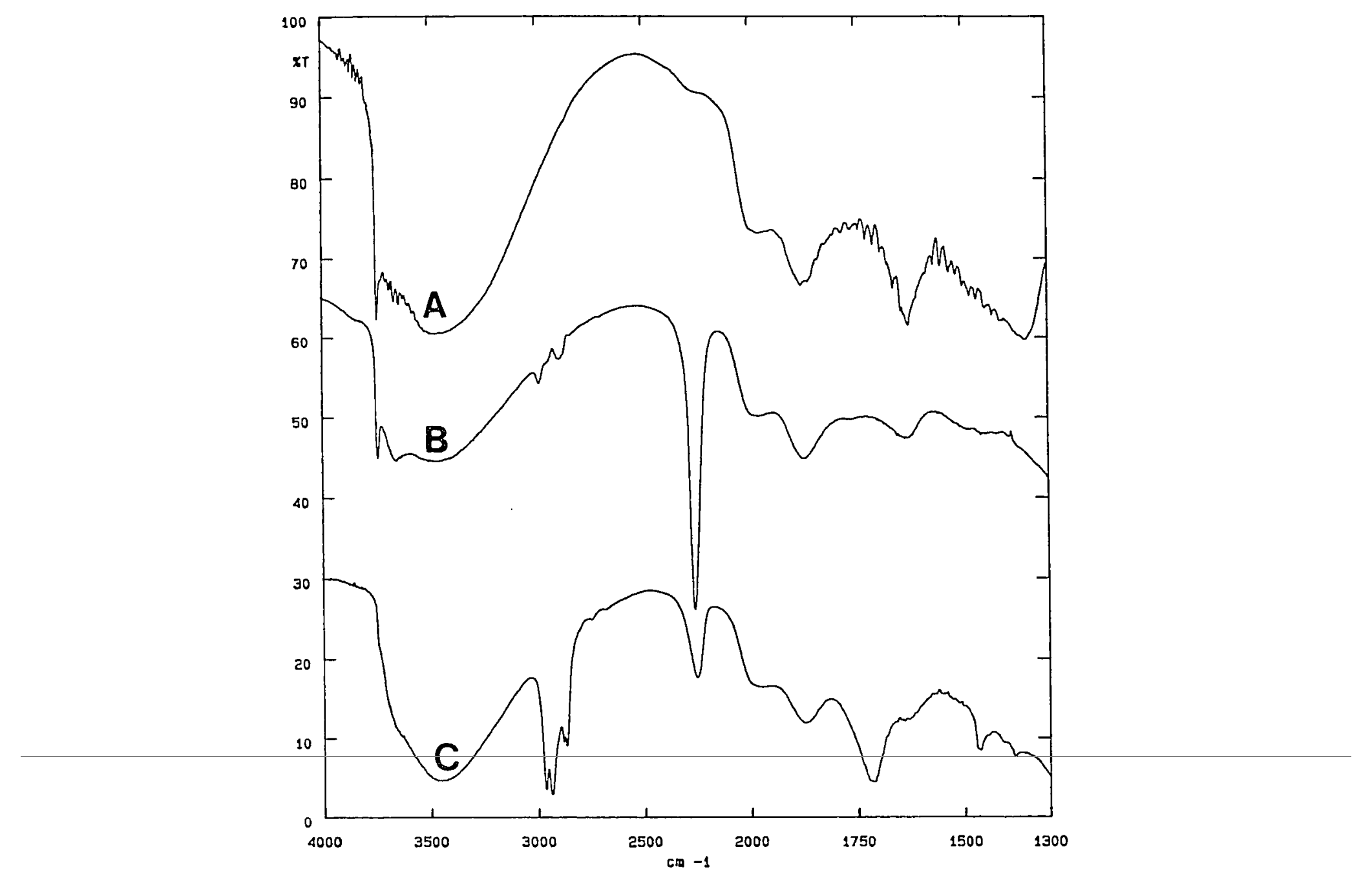

Figure 47. Change in the Partial DRIFT Spectra of Rromasil Silica upon Derivatization (A) Native silica; (B) Hydride intermediate prepared via hydrosilanization; (c) Octyl-bonded phase. 


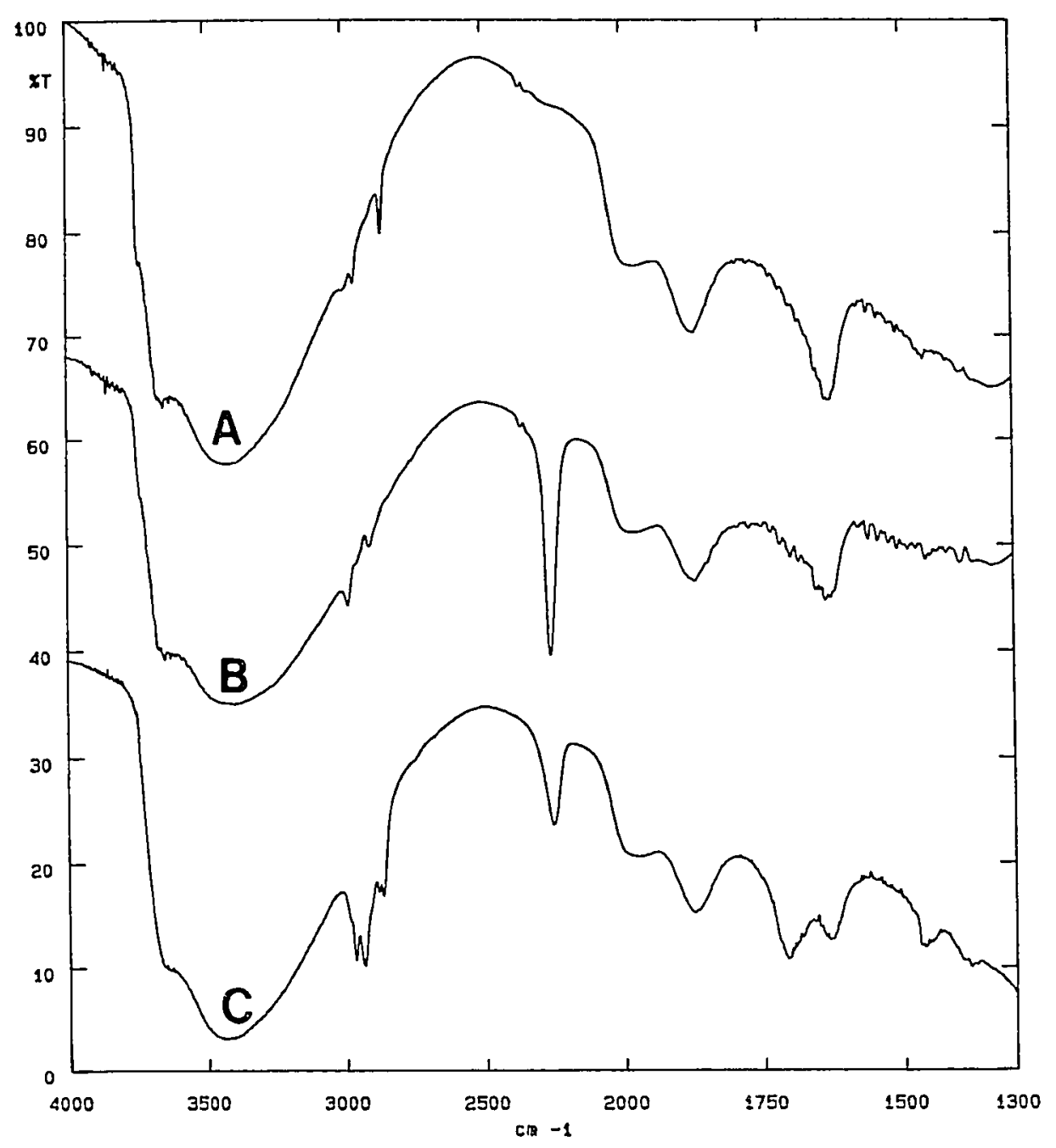

Figure 48. Change in the Partial DRIFT spectra of Vydac-Lot 890414 Silica upon Derivatization (A) Native silica; (B) Hydride intermediate prepared via hydrosilanization; (C) Octyl-bonded phase. 


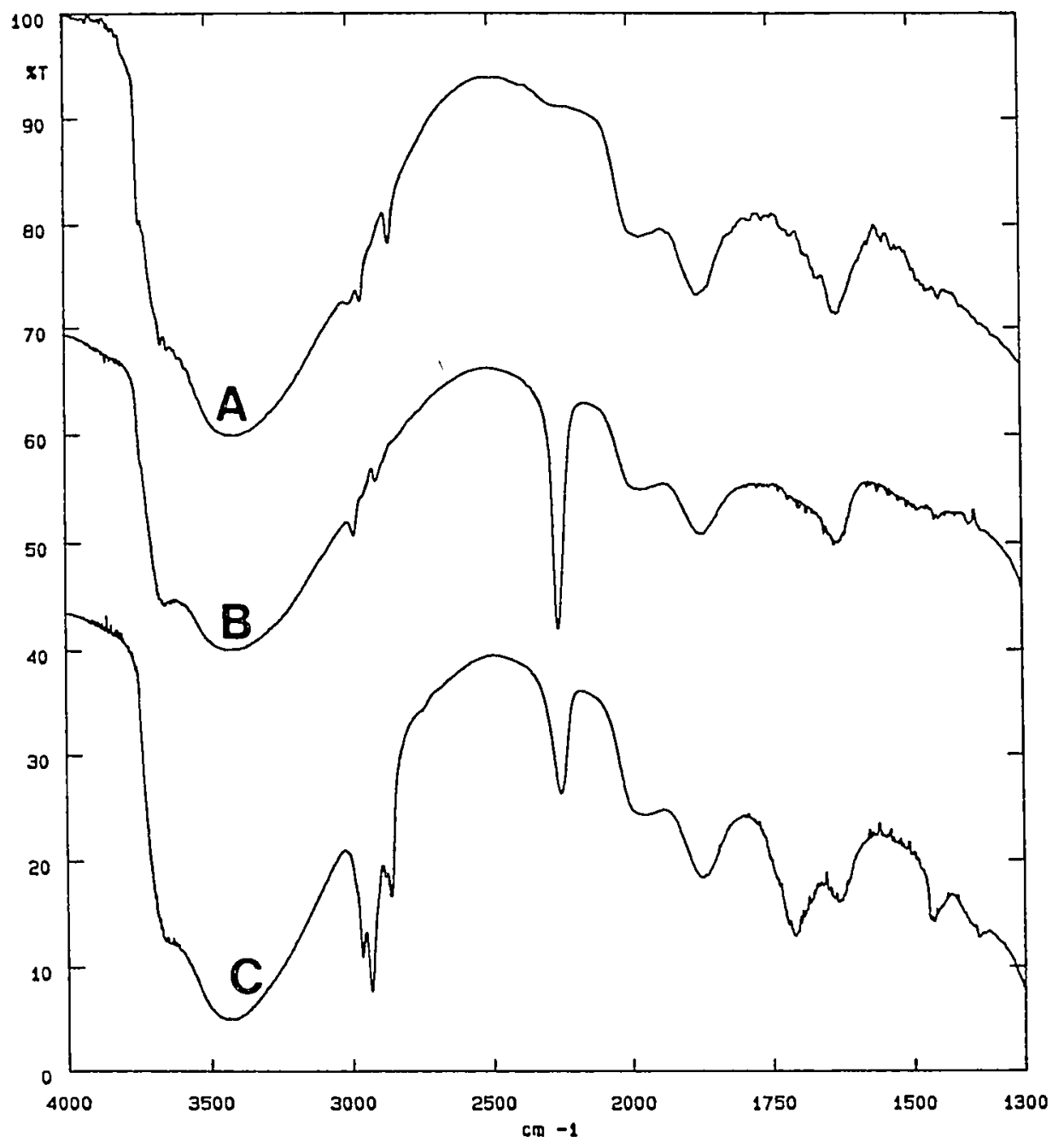

Figure 49. Change in the Partial DRIFT Spectra of vydac-Iot 890706 Silica upon Derivatization (A) Native silica; (B) Hydride intermediate prepared via hydrosilanization; (C) Octyl-bonded phase. 


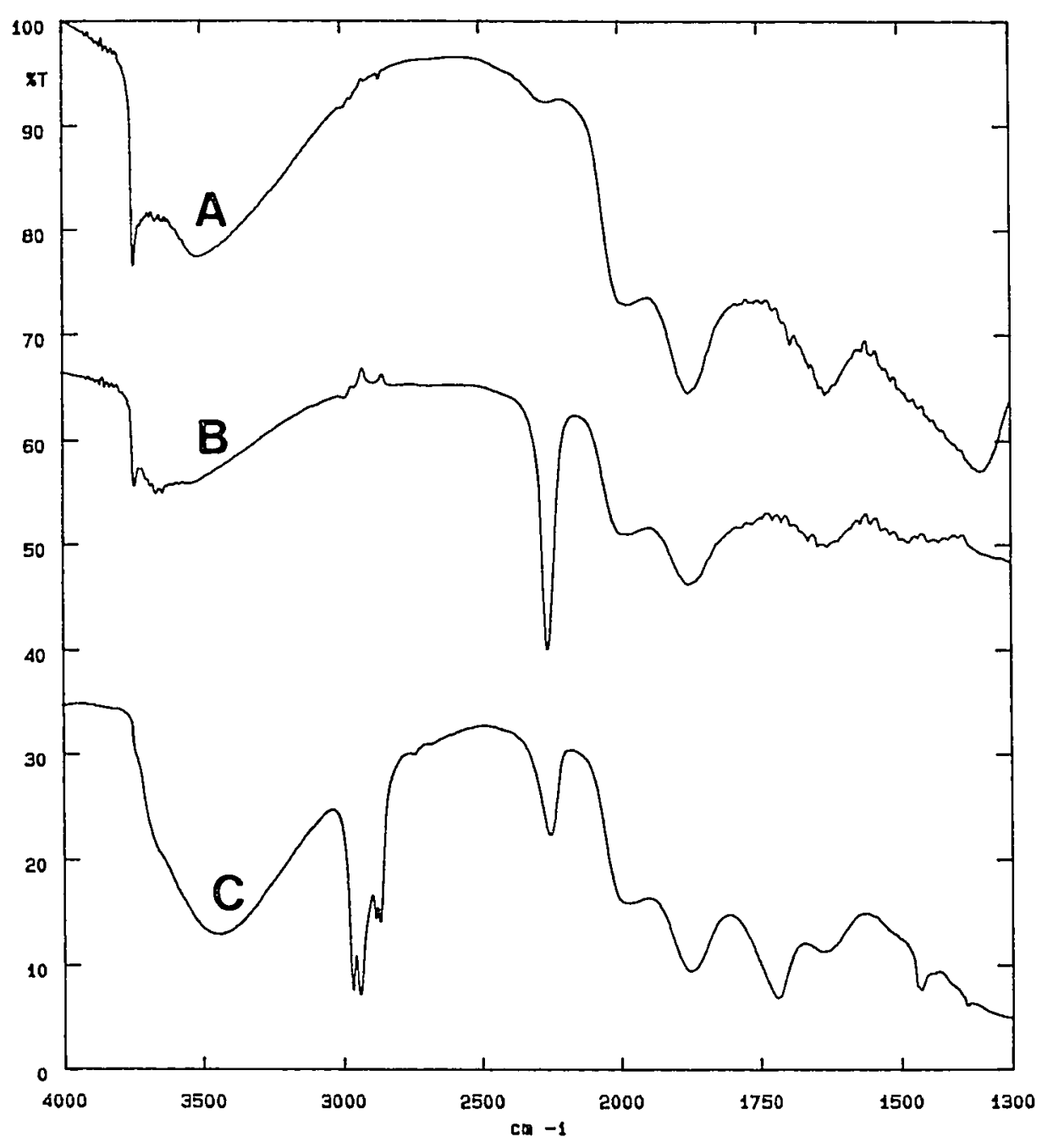

Figure 50. Change in the Partial DRIFT Spectra of Nucleosil Silica upon Derivatization (A) Native silica; (B) Hydride intermeãiate prepared via hydrosilanization; (C) Octyl-bonded phase. 
Table 10. Surface Coverage Properties of Octyl-silicas Prepared via Hydrosilation of 1-octene

\begin{tabular}{|c|c|c|c|c|c|}
\hline & c-Content & corrected $^{\mathrm{a}}$ & octyl & bverage & $\%$ \\
\hline Silica & $\% W t$ & $C, \% W t$ & $\mathrm{mmol} / \mathrm{g}$ & $\mu \mathrm{mol} / \mathrm{m}^{2}$ & Eff \\
\hline Partisil-40 & 10.45 & 9.94 & 8.3 & 3.04 & 30 \\
\hline Partisil-10 & 10.35 & 9.92 & 10.8 & 3.49 & 43 \\
\hline Kromasil-100A & 6.93 & 6.44 & 10.2 & 2.63 & 32 \\
\hline Vydac $(890414)$ & 3.89 & 2.98 & 60.1 & 3.88 & 27 \\
\hline Vydac $(890706)$ & 4.15 & 3.31 & 52.3 & 3.90 & 22 \\
\hline Nucleosil $300-10$ & 3.63 & 3.47 & 30.7 & 3.37 & 48 \\
\hline
\end{tabular}

a After substracting the amount present before bonding (last column of Table 9)

concomitant with a significant decrease of the intensity of the Si-H strecthing band at $2260 \mathrm{~cm}^{-1}$ clearly confirms bonding to the silica substrate.

One of the most important features of a bonded phase is its hydrolytic stability, particularly when used with aggressive chromatographic mobile phases. A particularly aggressive eluent is that containing an aqueous solution of trifluoroacetic acid (TFA). It has been shown that a rapid column degradation takes place when these mobile phases 
are used during the chromatographic separation of proteins (25). To estimate the extent of phase degradation, selected bonded silica samples were subjected to prolonged exposure to a solution containing $0.1 \% \mathrm{v} / \mathrm{v}$ of TFA and $20 \% \mathrm{v} / \mathrm{v}$ dioxane (17). The latter was used to improve the support's wetability and thus a thorough contact with the bonded support could be readily achieved. The amount of remaining phase was obtained from carbon determinations on sample aliquots periodically taken from the hydrolysis flask. Figures 51 and 52 show that although all bonded silicas exhibit degradation, the extent of it was significantly lower for the hydrosilation product than that of the same support modified by conventional octyldimethyl-silanization (equation 1). It can be seen that at the end of the test, the conventional bonded product had lost about $50 \%$ of its initial coverage while the octyl-silicas prepared via 1-octene hydrosilation lost only about $15 \%$ of its starting coverage. These simple long-term hydrolysis experiments clearly demonstrate the superior hydrolytic stability of Si-C linkages as compared to that of Si-O-Si-C linkages present in currently used bonded silicas. 


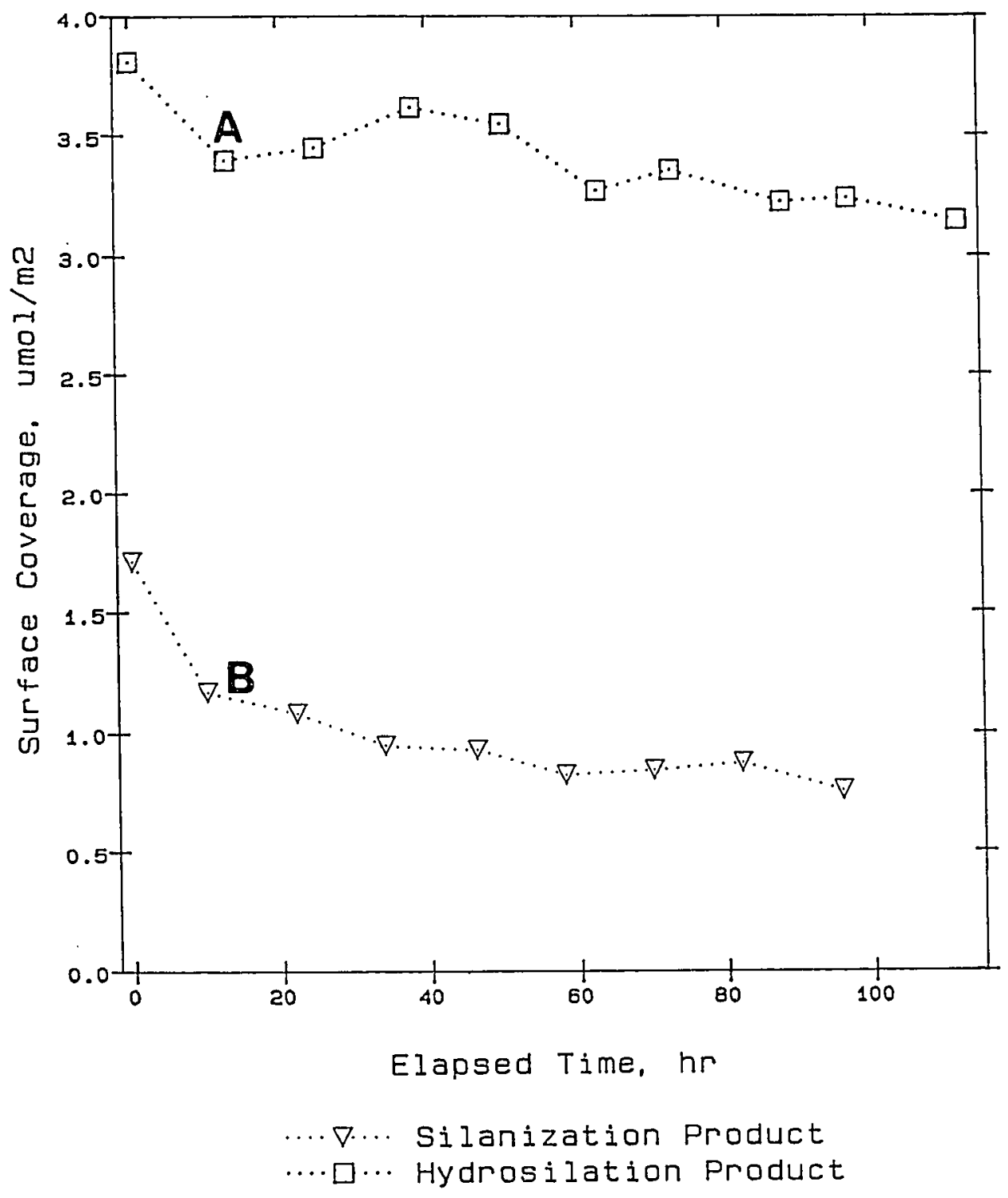

Figure 51. Surface Coverage of Octyl-bonded Vydac Silica as a Function of Hydrolysis Time: Prolonged treatment was at room temperature in $0.1 \% \mathrm{~V} / \mathrm{V}$ TFA aqueous solution containing $20 \%$ $\mathrm{v} / \mathrm{v}$ of dioxane. (A) Octyl-silica prepared by hydrosilation; (B) Octyldimethylsilyl-silica by silanization from a commercial procedure 


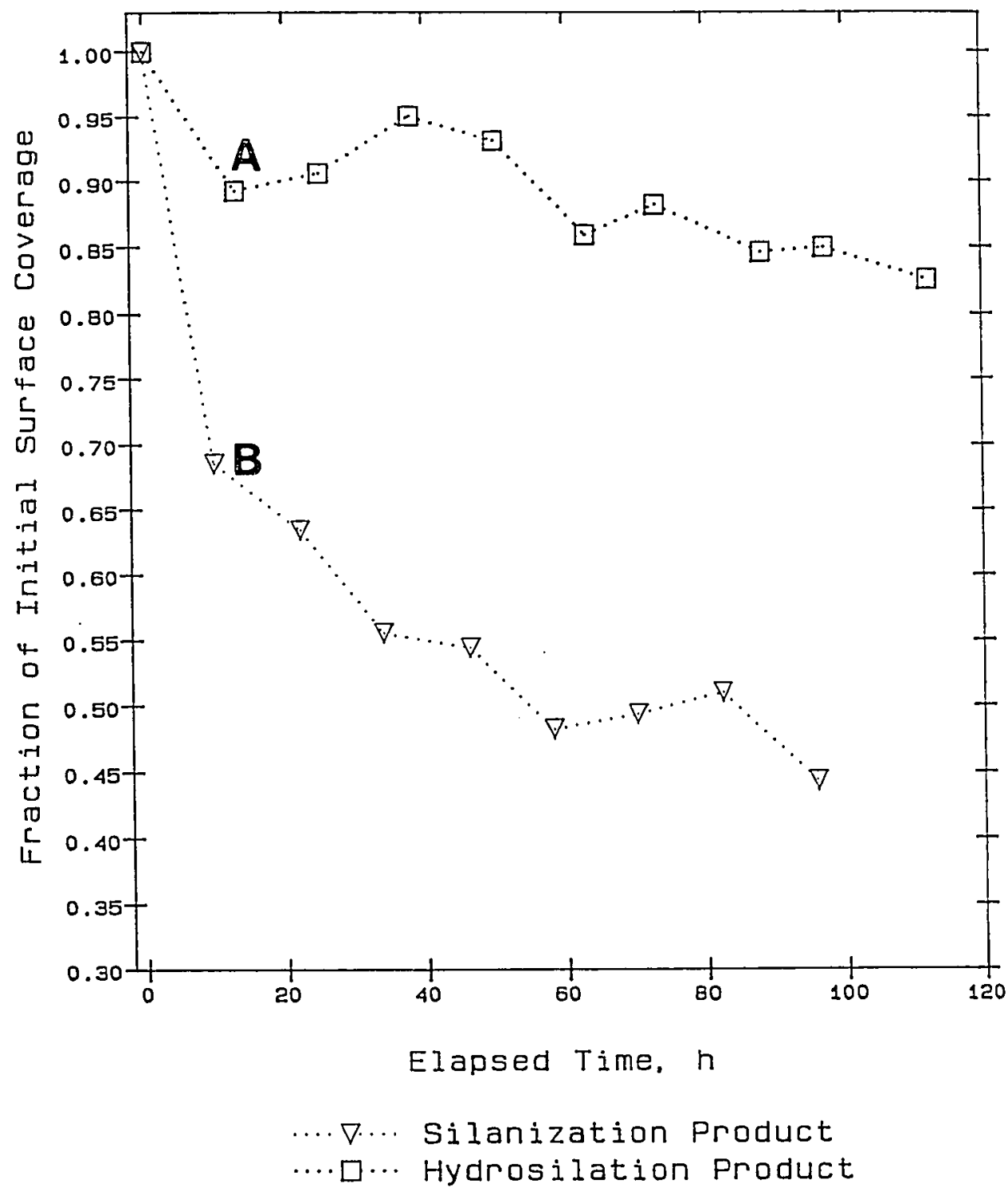

Figure 52. Relative Coverage as a Function of Hydrolysis Time. Curves designation as in Figure 51 


\section{CONCLUSIONS}

The merits and limitations of silica hydrosilanization with TES as an alternate method to prepare hydride-modified silicas are presented in this section. Additionally, specific avenues for further work are pointed out.

Generally speaking, the primary goals of this project have been met: compared to the chlorination/reduction sequence (7), hydrosilanization with TES does indeed provide a simpler and more efficient approach to prepare a hydridemodified silica substrate.

A working set of experimental conditions for TES-hydrosilanization is as fallows:

- Solvent: Dioxane or similar aprotic solvent.

- Amount of TES: 10-40\% molar excess with respect to available silanols.

- Acid Catalyst: Aqueous $\mathrm{HCl}$ with a concentration of $0.1 \mathrm{M}$ or above.

- Reaction Time: 40-60 min.

- Reaction Temperature: That for a gentle reflux. Under these conditions, TES-hydrosilation provides $\mathrm{SiH}$ coverage efficiencies of about $80-100 \%$ (with respect to truly available silanols). This represents about a 5-fold improvement over the chlorination/reduction approach.

Besides improving hydrosilane coverage, the new proce- 
dure effectively eliminates the strictly-dry condition required by the chlorination/reduction method. In fact, the presence of water is required to hydrolyze the ethoxy groups of TES into silanols. Furthermore, the potential hazard associated with aluminum hydride byproducts (7) is completeIy eliminated. As a result, TES-hydrosilanization is simpler and more economical to carry out than the chlorination/reduction counterpart: (i) consumption of reagents and solvents is greatly reduced; (ii) at the bench level, process time is reduced from $3-4$ full days to about half a day; (iii) use of man-power is drastically reduced.

From an operational point of view, when scaled up, TEShydrosilanization does not require additional equipment: the same industrial infrastructure currently available for organosilanization can readily be used for TES-hydrosilanization as well as for olefin-hydrosilation.

Due to its simplicity, ease of carrying out, enhanced $\mathrm{SiH}$ coverage and freedom from dry-conditions, it is clear from the results of this study that hydrosilanization with TES should replace the chlorination/reduction as a means to produce hydride-modified silica substrates.

The issue of $\mathrm{SiH}$ monolayer formation is, however, more difficult to assess. Results from DRIFT, DSC/air, TGA and $\mathrm{H}_{2}$-evolution/GC are not straight forward in evaluating the extent of an eventual multilayer formation. We have used 
DRIFT, DSC and to a lesser extent TGA to indirectly evaluate the "purity" of the chemisorbed SiH species. For instance, we have attributed DSC oxidation peaks broadening of several samples to the contribution from polymeric materials of the type $\left(\mathrm{HSiO}_{3 / 2}\right)_{\mathrm{n}}$. However, an unequivocal assignment of such species has not been obtained at this point. Because of their extreme sensitivity to molecular structure, modern solid NMR techniques such as ${ }^{29} \mathrm{Si}$ CP-MAS (cross polarization-magic angle spinning) and perhaps ${ }^{I_{H}}$ CRAMPS (combined rotation and multiple-pulse spectroscopy) seem particularly well suited for evaluating $\mathrm{SiH}$ monolayer formation. These NMR techniques alone or combined with DRIFT and/or DSC should provide a more complete qualitative and quantitative evaluation of the hydrided silica surface.

Extension of TES-hydrosilanization to other inorganic oxide substrates is an important application of the procedures described in this work. Porous materials such as alumina, zirconia and titania, among others, are amenable of being modified with a monolayer of hydrosilane. Further modification by olefin hydrosilation should provide a variety of separation materials for chromatograpic use. 


\section{BIBLIOGRAPHY}

1. Unger, K. K. Porous Silica.- Its Properties and Use as Support in Column Liquid Chromatography (Journal of Chromatography Library, Vol. 16); Elsevier Scientific Publishing Co.: New York, 1979.

2. Kohler, J.; Chase, D. B.; Farlee, R. D.; Vega, A. J.; Kirkland, J. J. J. Chromatogr. 1986, 352, 275-305.

3. Kirkland, J. J.; McCormick, R. M. Chromatographia. 1987, $24,58-76$.

4. Sander, L. C.; Wise, S. A. anal. Chem. 1984, 56, 504-510.

5. Unger, V. K.; Thomas, W.; Adrian, P. Kolloid-Z. 1973, $251,45-52$.

6. Pesek, J. J.; Swedberg, S. A. J. Chromatogr. 1986, $361,83-92$.

7. Sandoval, J. E.; Pesek, J. J. Anal. Chem. 1989, 61, 20672075 .

8. Budkevich, G. B.; Slinyakova, I. B.; Neimark, I.E. USSR Pat. 215884, 1965 .

9. Slinyakova, I. B.; Budkevich, G. B.; Neimark, I. E. Kolloidn. Zh. 1965, 27, 758-764。

10. Budkevich, G. B.; Slinyakova, I. B.; Neimark, I. E. Kolloidn. Zh. 1966, 28, 21-26.

11. Slinyakova, I. B.; Kurennaya, L. I.; Neimark, I. E. Fiz.-Khim. Mekh. Liofil'nost Dispersnykh Sist. 1971, 
No. $2,313-319$.

12. Budkevich, G. B.; Slinyakova, I. B.; Neimark, I. E.; Zhaigailo, Y. V. Ukr. Khim. Zh. 1971, 37, 429-433.

13. Kurennaya, L. I.; Slinyakova, I. B. Kolloidn. Zh. $1975,37(1), 178-181$.

14. Morterra, C.; Low, M. J. D, J. Phys. Chem. 1969, 73, $321-326$.

15. Morterra, C.; Low, M. J. D, J. Phys. Chem. 1969, 73, $327-333$.

16. Iler, R. K. The Chemistry of silica. - Solubility, Polymerization, colloid and surface Properties, and Biochemistry; John Wiley \& Sons: New York, 1979.

17. Sandoval, J.E.; Pesek, J.J. Submitted to Anal. Chem.

18. Technical Committee of Welding Electrodes, Diffusible Hydrogen in Mild Steel and Low-Alloy Steel Weld Metals: Test Method W 487 - M 1977, 1977.

19. Smith, A. L. Analysis of Silicones; John Wiley \& sons: New York, 1974.

20. Plueddemann, E. P. Silane coupling Reagents; Plenum Press, New York, 1982.

21. Freund, F.; Masuda, M. M.; Freund, M. M. submitted to J. Mat. Res.

22. Berendsen, G. E.; De Galan, I. J. Liq. Chromatogr. 1978. 1, 561-586.

23. Kinkel, J. N.; Unger, K. K. J. Chromatogr. 1984, 316, 
$193-200$

24. Cheng, W.; McCown, M. J. Chromatogr. 1985. 318, 173-185.

25. Glajch, J. L.; Kirkland, J. J.; Kohler, J. J. Chromatogr. 1987, 384, 81-90.

26. West, R. J.Am. Chem. Soc. 1954, 76, 6015-6017. 


\section{APPENDIX}

6.1. Significance of Hydrosilane Coverage in a Hydrideintermediate support

Similarly to other surface modified products, the extent of surface coverage of attached groups on silica is an important parameter which directly measures the efficiency (yield) of the modification procedure(s). For instance, the surface group density in bonded reversed phases largely determines their separation performance (1). Determination of surface alkyl coverage $(\mathrm{mmol} / \mathrm{g})$ is generally carried out by elemental analysis. The utilization of this approach for hydrosilane coverage measurements on hydride silica has the inconvenience of interferences from other hydrogen-containing species present in the support (namely, physically adsorbed water and surface silanols).

Spectroscopic evidence such as DRIFT and NMR have being routinely used for the structural characterization of silica and its derivatives. Similarly, the thermooxidative DSC and TGA behavior of the SiH species has been the basis for further characterization of polyhydrosiloxane xerogel (12) as well as hydride-modified silica (7). Although they are extremely powerful analytical tools, the forementioned techniques provide, at best, semi-quantitative information 
about relative changes of the surface species. Major Iimitations which prohibit their use for quantitative purposes include lack of appropriate standards (all mentioned techniques), poor linear dynamic range (IR), as well as difficulties associated with reproducibility in sample preparation (IR and NMR).

Several "wet" analytical methods have been developed and applied on SiH-containing materials to determine their hydrosilane content (19). Many of such methods exploit the well-known ability of hydrosilane species to quantitatively reduce certain metallic ions such as Pd(II), Pt(II), Ag(I) and $\mathrm{Hg}(\mathrm{II})(10)$. When a hydride-modified silica was treated with a stoichiometric excess of a $\mathrm{Pd}$ (II) solution, Sandoval and Pesek (7) found that only partial reaction of the surface $\mathrm{SiH}$ species was attained even after a prolonged treatment of the support with the metal solution. The negative bias of the determination was attributed to "the accumulative deposition of finely dispersed metallic particles which eventually clogged the porous and physically blocked further interaction of internal $\mathrm{SiH}$ species with metallic ions from the bulk liquid " (7). Similar results were obtained with Pt(II) and Ag(I) solutions.

The necessity still exists for a suitable approach to quantitatively determine the extent at which the silica surface is covered by silicon hydride species. Hydrosilane 
coverage directly affects the extent of hydrosilation (the final bonding process) because the SiH species provide all the active sites for olefin addition (equation 5 ).

\subsection{Hydrogen Displacement Methods}

When a hydrosilane-containing compound is subjected to alkaline hydrolysis, quantitative generation of hydrogen-gas results, according to the equation (26)

$$
\begin{aligned}
& \mathrm{OH}^{-} \\
& \equiv \mathrm{Si}-\mathrm{H}+\mathrm{H}_{2} \mathrm{O}-\cdots-\mathrm{Si}-\mathrm{OH}+\mathrm{H}_{2} \\
& \text { Catalyst }
\end{aligned}
$$

The reaction is well suited for hydrosilane quantitation when an adequate method to collect and quantate the gas is available. Because of its great simplicity, a volumetrically-calibrated glass tube was used with this purpose. Such a gas collecting apparatus is depicted in Figure 1. When applied to hydrosilanized substrates, the precision was about 15\% relative standard deviation (RSD), but frequently was as large as $30 \%$. Obviously, this level of uncertainty would preclude a meaningful evaluation of the different factors affecting hydrosilanization with TES. The poor 
precision of the gas evolution/volumentry method can be attributed to several factors, mostly arising from sample handling. Eventhough extreme care was taken to avoid the inclusion of any air bubble during the tube filling process, some air might have been trapped in the porous matrix of the substrate. Also, some floating sample might be lost during the filling of the vial with the acidic solution. In general, the analytical set-up procedures of the method appeared to be extremely demanding with respect to the analyst's manual skills. The method was therefore abandoned.

A different method was then used to measure the amount of $\mathrm{H}_{2}$-gas evolved during the alkaline hydrolysis of the hydrosilanized samples. In this case, the gas was collected in a syringe and injected into a GC instrument which was calibrated against a hydrosilane standard. The method used was adapted from procedures developed by Smith (19) . A significantly more efficient hydrogen recovery was possible since the gas was confined within hermetically sealed vials and special gas-tight syringes. Additionally, since the gas sample was chamatographically separated, any air trapped within the syringe did not interfere with quantitation. It should be point out that special care had to be devoted to the choice of a vial septum which provided a truly gas-tight contact with the syringe's needle. After several tries, a neoprene septum ("Blue Septa", Altech Associates, Deerfied, 
Il.) was found appropriate.

The overall analytical precision for the $\mathrm{H}_{2}-$ evolution/GC method was about 2-4\% RSD. Although almost as demanding as gas volumetry, GC-quantitation was much more reliable. Triphenylsilane was used to standardize the GCinstrument. Its exact $\mathrm{siH}$ content was determined by means of a redox titration method (19). The latter is based on the reduction of mercury (II) with the hydrosilane, according to the equation

$2 \mathrm{Hg}(\mathrm{OAC})_{2}+\equiv \mathrm{SiH}------>\mathrm{Hg}_{2}(\mathrm{OAC})_{2}+\equiv \mathrm{SiOMe}+2 \mathrm{HOAC}$ (19)

The released acid is then titrated with alcoholic koH with phenolphthalein as indicator. The triphenylsilane reagent used was found to contain $3.93 \pm 0.04 \mathrm{mmol} / \mathrm{g}$, a value which corresponds to a composition of $102.4 \%$ as $\left(\mathrm{C}_{6} \mathrm{H}_{5}\right)_{3} \mathrm{SiH}$.

6.3. Correlation Between Hydrosilane Coverage and DSC/Air Data

It was stated in section 3.1 and 3.2 .1 (p. 24 and 46) that the DSC peak area for the thermooxidation of SiH groups (equation 15) increases with the population of these species on the silica surface. A plot of the DSC/air enthatpy $(\triangle H$, as measured by the peak area) versus the specific SiH cover- 
age ( $\mathrm{A}_{\mathrm{SiH}}$, in mmol/g), shown in Figure 53, clearly indicates that the results obtain with each technique are highly correlated. The fitting data shown (slope and intercept) can be used to obtained a rough estimate of hydrosilane coverage from DSC/air measurement, the latter having the advantage of not requiring any sample manipulation other than loading in the instruments' pan. An intercept statistically higher than zero can be attributed to the contribution to the correlation from tailing DSC/air peaks which, as mentioned before (section 3.2 .1$, P. 46) tend to show a negatively biased enthalpy measurement (see curves $A-D$ of Figure 22). The validity of this argument can be tested by correlating the logarithms of $\triangle H$ and $A_{S i H} \cdot A$ perfectly linear correlation should result in a slope equal to unity:

$$
\log \Delta \mathrm{H}=\log \mathrm{K}+\log \mathrm{A}_{\mathrm{SiH}}
$$

where $\mathrm{K}$ is the corresponding proportionality constant. A resultant slope value of $0.773 \pm 0.043$ is clearly statistically smaller than unity and, therefore, confines the contribution of tailing peaks to the correlation. As a consequence, any estimate of $\mathrm{A}_{\mathrm{SiH}}$ from a calibration plot such as that in Figure 53 should be used with caution and, when accuracy is mandatory, must be confirmed by a direct measurement using the $\mathrm{H}_{2}$-evolution/GC method. 


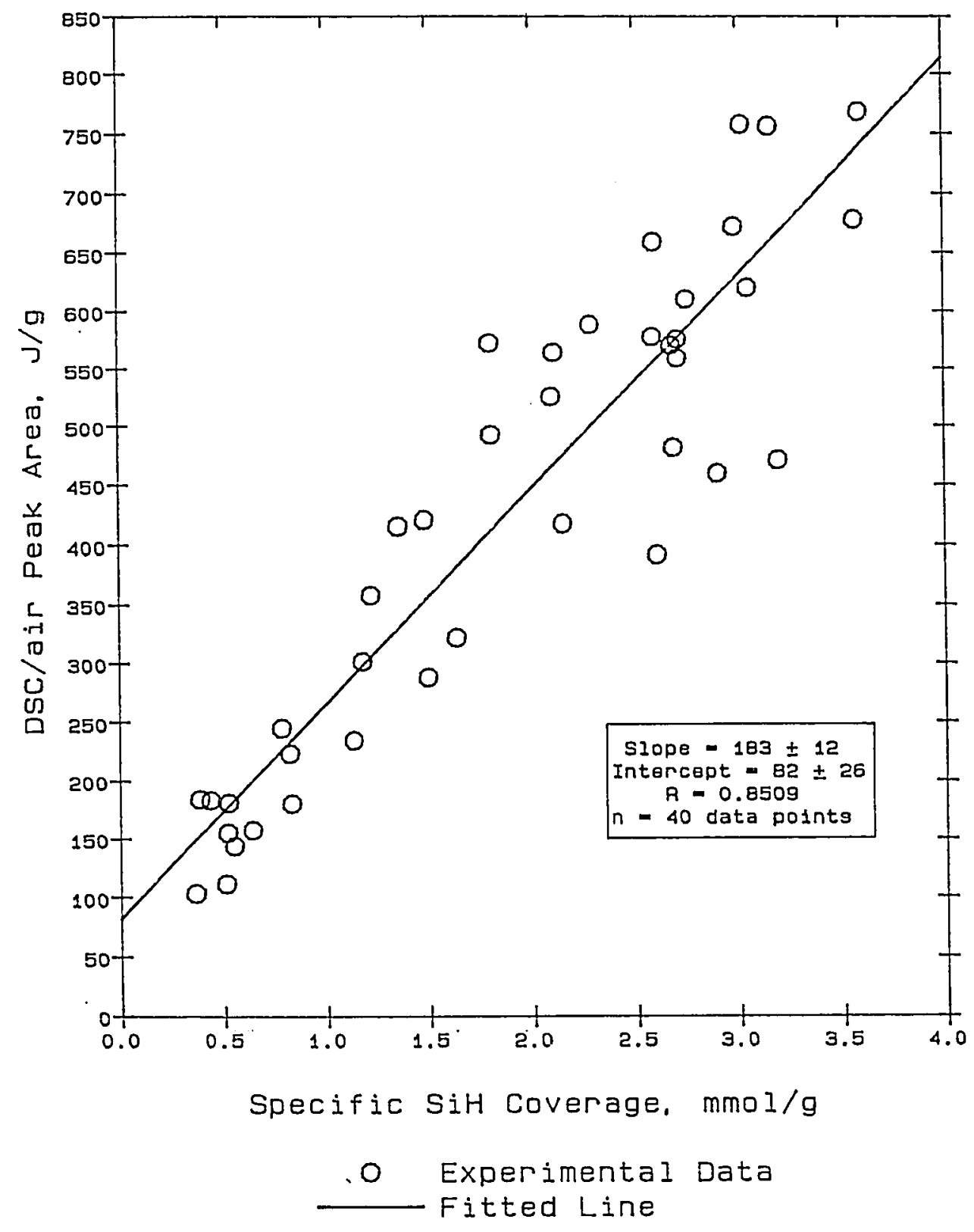

Figure 53. Correlation Between DSC/Air Data and Specific Hydrosilane Coverage 